\title{
Shortcuts to adiabaticity: Concepts, methods, and applications
}

\author{
D. Guéry-Odelin \\ Laboratoire de Collisions Agrégats Réactivité, CNRS UMR 5589, IRSAMC, \\ Université de Toulouse (UPS), 118 Route de Narbonne, \\ 31062 Toulouse CEDEX 4, France
}

A. Ruschhaupt and A. Kiely
Department of Physics, University College Cork, Cork, Ireland

E. Torrontegui

Instituto de Física Fundamental IFF-CSIC, Calle Serrano 113b, 28006 Madrid, Spain

\author{
S. Martínez-Garaot and J. G. Muga \\ Departamento de Química Física, UPV/EHU, Apdo. 644, 48080 Bilbao, Spain
}

(published 24 October 2019)

\begin{abstract}
Shortcuts to adiabaticity (STA) are fast routes to the final results of slow, adiabatic changes of the controlling parameters of a system. The shortcuts are designed by a set of analytical and numerical methods suitable for different systems and conditions. A motivation to apply STA methods to quantum systems is to manipulate them on timescales shorter than decoherence times. Thus shortcuts to adiabaticity have become instrumental in preparing and driving internal and motional states in atomic, molecular, and solid-state physics. Applications range from information transfer and processing based on gates or analog paradigms to interferometry and metrology. The multiplicity of STA paths for the controlling parameters may be used to enhance robustness versus noise and perturbations or to optimize relevant variables. Since adiabaticity is a widespread phenomenon, STA methods also extended beyond the quantum world to optical devices, classical mechanical systems, and statistical physics. Shortcuts to adiabaticity combine well with other concepts and techniques, in particular, with optimal control theory, and pose fundamental scientific and engineering questions such as finding speed limits, quantifying the third law, or determining process energy costs and efficiencies. Concepts, methods, and applications of shortcuts to adiabaticity are reviewed and promising prospects are outlined, as well as open questions and challenges ahead.
\end{abstract}

DOI: 10.1103/RevModPhys.91.045001

\section{CONTENTS}

I. Introduction

A. Overview of shortcuts to adiabaticity

B. Motivation and scope of this review II. Methods

A. Overview of inverse engineering approaches

1. Quantum transport

2. Spin manipulation

3. Beyond mean values

B. Counterdiabatic driving

1. Superadiabatic iterations

2. Beyond the basic formalism

a. "Physical" unitary transformations

b. Schemes that focus on one state

c. Effective counterdiabatic field

d. Dressed-states approach

e. Variational approach

f. Counterdiabatic Born-Oppenheimer dynamics

g. Constant CD-term approximation

C. Invariants and scaling laws

1. Lewis-Riesenfeld invariants
2. Examples of invariant-based inverse engineering 13
a. Two-level system

b. Lewis-Leach family

3. Scaling laws

4. Connection with Lax pairs

D. Variational methods

E. Fast forward

1. The original formalism

2. Streamlined fast-forward approach

3. Generalizations and terminology

F. FAQUAD and related approaches

G. Optimal control and shortcuts to adiabaticity

H. Robustness

1. Error sensitivity and its optimization using perturbation theory

a. Illustrative example: Control of a two-level system

b. Optimization using perturbation theory in other settings

2. Other approaches 23

I. Three-level systems 
1. Apply counterdiabatic shortcuts to the full three-level $\Lambda$ system

2. Applying invariant-based inverse engineering shortcuts to the full three-level $\Lambda$ system

3. Using STA techniques after mapping to a two-level system

J. Motional states mapped into a discrete system

III. Applications in Quantum Science and Technology

A. Trapped ions

1. Dynamical normal modes

2. Ion transport

3. Other operations

B. Double wells

C. Cavity quantum electrodynamics

D. Superconducting circuits

E. Spin-orbit coupling

F. Nitrogen-vacancy centers

G. Many-body and spin-chain models

H. Metrology

IV. The Energy Cost of STA, Engines, and the Third Law

A. Energy costs

B. Engines and refrigerators

C. Third law

V. Open Quantum Systems

A. Concept of adiabaticity for open systems

B. Engineering the environment

C. Mitigating the effect of environment

D. Non-Hermitian Hamiltonians

VI. Optical Devices

VII. Extension to Classical and Statistical Physics

A. Counterdiabatic methods in classical mechanics

B. Mechanical engineering

1. Cranes

2. Robustness issues

C. STA for isolated dilute gases

1. Boltzmann equation

2. Extension to Navier-Stokes equation

D. Shortcuts for systems in contact with a thermostat

1. The overdamped regime

2. Connection with free energy and irreversible work

3. Extensions

VIII. Outlook and Open Questions

List of Symbols and Abbreviations

Acknowledgments

Appendix A: Example of Lie Transform

Appendix B: Counterdiabatic Hamiltonian for a Two-level

References

$$
\text { Hamiltonian with Complex Coupling }
$$

\section{INTRODUCTION}

\section{A. Overview of shortcuts to adiabaticity}

"Shortcuts to adiabaticity" (STA) are fast routes to the final results of slow, adiabatic changes of the controlling parameters of a system. Adiabatic processes are here broadly defined as those for which the slow changes of the controls leave some dynamical properties invariant, the "adiabatic invariants," such as the quantum number in quantum systems or phasespace areas in classical systems. Figure 1 informally portrays the central idea of shortcuts to adiabaticity. Figure 2 also

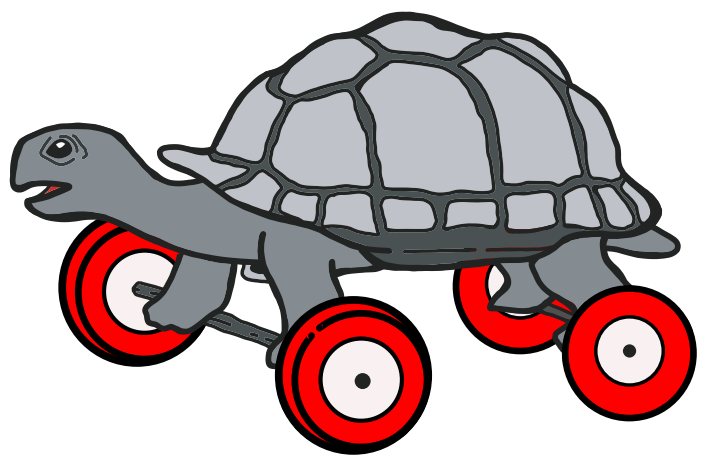

FIG. 1. A turtle on wheels is a good metaphor for shortcuts to adiabaticity. The image is inspired by the artist work of Andree Richmond.

illustrates the concept pictorially by comparing diabatic, adiabatic, and STA paths in a discrete system.

The shortcuts rely on specific time dependences of the control parameters and/or on the addition of auxiliary timedependent couplings or interactions with respect to some reference Hamiltonian or, more generally, a Liouvillian or transition-rate matrix. STA methods were first applied in simple quantum systems: two- and three-level systems or a particle in a time-dependent harmonic oscillator. They have since come to encompass a much broader domain since slow processes are quite common as a simple way to prepare the state of a system or to change conditions avoiding excitations in a wide spectrum of areas from atomic, molecular, and optical physics, solid state, or chemistry to classical mechanical systems and engineering. In parallel to such a large scope, different methods have been developed and applied.

This description of shortcuts needs some caveats and clarifications. In many quantum mechanical applications, the final state in an STA process reproduces the set of adiabatic probabilities to find the system in the eigenstates of the final Hamiltonian. The mapping of probabilities from

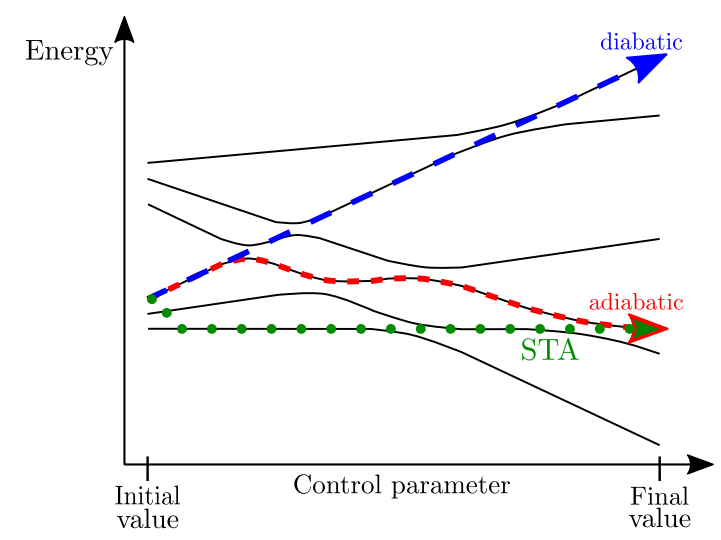

FIG. 2. Schematic example of adiabatic, diabatic, and STA processes. The system is initially in the third level. In the adiabatic path (short-dashed red) the system evolves always along the third level. In a diabatic evolution (long-dashed blue) the system gets excited by jumping across avoided crossings. Along an STA path (green dots) the system does not always travel along the third level but it arrives at the third level in a shorter time (the time dimension is not explicitly shown in the figure). 
initial to final settings, without final excitation but allowing for excitations en route, holds for any possible initial state in some state-independent STA protocols and simple enough systems. These state-independent shortcuts are ideal for maximal robustness and to reduce dependence on temperature, so as to avoid costly and time-consuming cooling to the ground state. However, except for idealized models, state independence holds only approximately and for a domain of parameters, for example, within the harmonic approximation in a trapped ion. In other applications state independence is not really necessary or it may be difficult or impossible to achieve as in chaotic systems. Yet shortcuts may still be found for a chosen specific state, typically the ground state or for a state subspace.

A further caveat is that the scope of STA methods has in practice outstripped the original, pure aim in many applications. For example, these methods can be extended with minimal changes to drive general transitions, regardless of whether the initial and final states can be connected adiabatically, such as transitions where the initial state is an eigenstate of the initial Hamiltonian, whereas the final state is not an eigenstate of the final Hamiltonian. This broader perspective merges with inverse engineering methods of the Hamiltonian to achieve arbitrary transitions or unitary transformations.

Motivations.-There are different motivations for the speedup that depend on the setting. In optics, time is often substituted by length to quantify the rate of change so the shortcuts imply shorter, more compact optical devices. In mechanical engineering, we look for fast and safe protocols, say of robotic cranes, to enhance productivity. In microscopic quantum systems, slowness often implies decoherence, the accumulation of errors and perturbations, or even the escape of the system from its confinement. The shortcuts provide a useful toolbox to avoid or mitigate these problems and thus to develop quantum technologies. Moreover, with shorter process times experiments can be repeated more often to increase signal-to-noise ratios.

A generic and important feature of STA apart from the speed achieved is that there are typically many alternative routes for the control parameters, and this flexibility can be used to optimize physically relevant variables, for example, to minimize transient energy excitations and/or energy consumptions or to maximize robustness against perturbations.

Shortcuts to adiabaticity in quantum technologies.Indeed, shortcuts have been mostly developed and applied for quantum systems, and much of the current interest in STA is rooted in the quest for quantum technologies. STA combine well with the two main paradigms of quantum information processing.

- In the gate-based paradigm, shortcuts to adiabaticity contribute to improve and speed up gates or state preparations and to perform elementary operations like moving atoms leaving them unexcited. Shortcuts to adiabaticity may be applied to all physical platforms, such as trapped ions, cold atoms, nitrogen-vacancy ( $\mathrm{N} V$ ) centers, superconducting circuits, quantum dots, or atoms in cavities.

Apart from fighting decoherence, implementing "scalable" architectures toward larger quantum systems is a second major challenge to develop quantum information processing. Scalability benefits from STA in two ways: directly, through the design of operations explicitly intended to achieve it (see an example in Sec. III.A), as well as indirectly, since a mitigated decoherence reduces the need for highly demanding, qubit-consuming error-correction codes.

- The second main paradigm of quantum information processing is adiabatic computing or quantum annealing, which may be accelerated or made possible by changing initial and/or final Hamiltonians, modifying the interpolation path between initial and final Hamiltonians, or adding auxiliary terms to the transient Hamiltonian. For adiabatic quantum computing and other applications with complex systems, progress is being made to find effective STA without using difficult-to-find information on spectra and eigenstates. The more recent paradigms of topological quantum information processing or measured based quantum computation may also benefit from STA.

Shortcuts to adiabaticity are also having an impact or are expected to be useful in quantum technologies other than quantum information processing such as interferometry and metrology, communications, and microengines or refrigerators in quantum thermodynamics.

Fundamental questions.-Finally, apart from being a practical aid to process design, STA also played a role and will continue to be instrumental in clarifying fundamental concepts such as quantum-classical relations, quantum speed limits and trade-off relations between timing, energy, robustness, entropy or information, the third law of thermodynamics, and the proper characterization of energy costs of processes.

\section{B. Motivation and scope of this review}

This review provides a broad overview of concepts, methods, and applications of shortcuts to adiabaticity. A strong motivation for writing it is the breadth of systems and areas where STA are used. The hope is that this work will assist in stimulating discussions and information transfer between different domains. There are already examples that demonstrate the benefits of such an interaction. Analogies found between systems as disparate as individual ions in Paul traps and mechanical cranes or optical waveguide devices and atomic internal states have proved fruitful. The link between Lewis-Riesenfeld invariants and Lax pairs is another unexpected example.

The development of STA methods and applications has been quite rapid since Chen, Ruschhaupt et al. (2010), where the expression shortcuts to adiabaticity was coined. Precedents before 2010 exist where STA concepts had been applied (Unanyan et al., 1997; Emmanouilidou et al., 2000; Couvert et al., 2008; Motzoi et al., 2009; Muga et al., 2009; Rezek et al., 2009; Salamon et al., 2009; Schmiedl et al., 2009; Masuda and Nakamura, 2010). In the post-2010 period, the early work of Demirplak and Rice (2003, 2005, 2008), and Berry (2009) on "counterdiabatic (CD) driving" has been particularly influential, as well as methodologies such as "inverse engineering," "invariants," "scaling laws," "fastforward," (FF) or "local adiabatic" methods. To add further flexibility to this already rich scenario of approaches, some methods provide in general a multiplicity of control protocols. Moreover, STA methods relate synergistically to or overlap partially with other control methods. In particular STA blend 
well with optimal control theory (OCT), decoherence-free subspaces (DFSs) (S. L. Wu et al., 2017), linear response theory (Acconcia, Bonança, and Deffner, 2015), or perturbative and variational schemes (see Secs. II.H and II.D). There is in summary a dense network of different approaches and STA protocols available, frequently hybridized.

Some of the basic STA techniques rely on specific formalisms (invariants and scaling, CD driving, or fastforward) that, within specific domains, can be related to each other and be made potentially equivalent because of underlying common structures. For example, a particular protocol to speed up the transport of a particle by defining a trap path may be found as the result of "invariant-based engineering," fastforward, or "local-CD" approaches. This convergence for specific operations and methods might be misleading because it does not extend to all systems and circumstances. No single all-inclusive theory exists for all STA, and each of the major methodologies carries different limitations, different construction recipes, and different natural application domains. A widespread misconception is to identify "STA" with one particular approach, often CD driving, and even with one particular protocol within one approach. We shall indeed pay due attention to unifying concepts and connections among the STA formalisms, which are of course useful and worth stressing, but at the same time it is hard to overemphasize that the diversity of approaches is a powerful asset of the shortcuts to adiabaticity that explains their versatility.

New hybrid or approximate methods are being created as we write and more will be devised to adapt to diverse needs and systems. This review is also intended to map and characterize the options, help users to navigate among them, and encourage the invention of novel, more efficient or goaladapted approaches. A number of techniques that fit naturally into the definition of STA but have not been usually tagged as STA will also be mentioned to promote transfer of ideas. Examples of this are the derivative removal of adiabatic gate (DRAG) and weak anharmonicity with average Hamiltonian (WAHWAH) approaches to implement fast pulses free from spurious transitions in superconducting qubits (Motzoi et al., 2009; Schutjens et al., 2013).

Terminology.-Not surprisingly, the various approaches to STA have been described and used with inconsistent terminologies by different groups and communities. Indeed the rapid growth of STA-related work and its extension across many disciplines has brought up different uses for the same words ("superadiabaticity" and "fast forward" are clear examples of polysemic terms), and different expressions for the same concept or method (for example, "transitionless quantum driving" and "counterdiabatic approach"). This review is also intended to clarify some commonly found uses or expressions, making explicit our preference when the polysemy may lead to confusion.

Scope and related reviews. - We intend the review to be didactical for the noninitiated but also comprehensive so that the experts in the different subfields can find a good starting point for exploring other STA-related areas. Several recent reviews on partial aspects or overlapping topics are useful companions: Torrontegui, Ibáñez et al. (2013) is the previous most comprehensive review on the subject, but the number of new applications in previously unexplored fields, experiments, and theoretical results since its publication clearly surpasses the work reviewed there. We mostly pay attention to the work done after Torrontegui, Ibáñez et al. (2013), but for presenting the key concepts some overlap is allowed. Work on the counterdiabatic method was reviewed recently by Kolodrubetz et al. (2017) with emphasis on geometric aspects and classical-quantum relations. For the approaches to speed up adiabatic computing, see Albash and Lidar (2018) and Takahashi (2019). For a review on STA to control quantum critical dynamics, see del Campo and Sengupta (2015). See also Menchon-Enrich et al. (2016) about the spatial adiabatic passage and Vitanov et al. (2017) about the stimulated Raman adiabatic passage (STIRAP), a technique that has often been sped up with STA. Finally, Masuda and Rice (2016) reviewed the counterdiabatic and fast-forward methods focusing on applications to polyatomic molecules and Bose-Einstein condensates (BEC). Also, a Focus issue of New Journal of Physics on Shortcuts to Adiabaticity provides a landscape of current tendencies (del Campo and Kim, 2019).

Structure.-The review is organized into "Methods" of STA in Sec. II and "Applications" from Sec. III to VII. We address applications of STA to quantum science and technology in Sec. III. This includes the main physical platforms and the quantum system is mainly considered as a closed quantum system. The connections between STA and quantum thermodynamic concepts, a topic between closed and open quantum systems, are reviewed in Sec. IV. STA and open quantum systems is the topic of Sec. V. Sections VI and VII deal with results and peculiarities of two fields that also offer a promising arena for practical applications: optics in Sec. VI and classical systems in Sec. VII.

We tried to keep acronyms to a minimum but some terms [among the most prominent: shortcuts to adiabaticity (STA) and counterdiabatic (CD)] appear so many times that the use of the acronym is well justified. Other acronyms are already of widespread use, such as OCT for optical control theory. To facilitate reading, a full list of acronyms is provided. As for notation, an effort toward consistency has been made, so the symbols often differ from the ones in the original papers. Beware of possible multiple uses of some symbols (constants, in particular). The context and text should help to avoid any confusion.

\section{METHODS}

\section{A. Overview of inverse engineering approaches}

We begin with an overview of inverse engineering. This expression refers to inferring the time variation of the control parameters from a chosen evolution of the physical system of interest. The notion is broadly applicable for quantum, classical, or stochastic dynamics and embraces many STA techniques. In this section we deal first with two simple examples for motional and internal degrees of freedom, where more sophisticated or auxiliary concepts and formalisms, e.g., related to invariants, counterdiabatic driving, or fast forward, are not explicitly used. Instead, the connection between dynamics and control is performed by solving for the control 
function(s) in the effective equation of motion for a classical variable or a quantum mean value.

It is often necessary or useful to go beyond this simple inversion approach for several reasons. For example: the dynamical path of the system is not easy to design, the inversion is nontrivial, we are interested in finding stateindependent protocols, the direct inversion leads to unrealizable control functions, or we look for stable controls, robust with respect to specific perturbations. To address these problems, in the following sections several auxiliary concepts and formal superstructures will be added to the simple inverse engineering idea in the different STA approaches. The first hint of a family of inversion methods dealing with detailed dynamics beyond mean values is given in Sec. II.A.3 after the two examples.

\section{Quantum transport}

Finding the motion of a harmonic trap to transport a quantum particle so that it starts in the ground state and ends up in the ground state of the displaced potential amounts to inversely solving a classical Newton equation (Schmiedl et al., 2009; Torrontegui et al., 2011). The equation of motion of the particle position $x$ inside the potential reads

$$
\ddot{x}+\omega_{0}^{2} x=\omega_{0}^{2} x_{0}(t),
$$

where $\omega_{0}$ is the angular frequency of the harmonic trap, $x_{0}(t)$ is the instantaneous position of its center, and the dots represent, here and in the following, time derivatives. Equation (1) describes a forced oscillator driven by the time-dependent force $F(t)=m \omega_{0}^{2} x_{0}(t)$. We can interpolate the trajectory $x(t)$ between the initial position of the particle and the desired final position $d$. In addition, to ensure that the transport ends up at the lowest energy state, one needs to cancel out the first and second derivatives at the initial $t=0$ and final time $t_{f}$ (Torrontegui et al., 2011). A simple polynomial interpolation of fifth degree can account for such boundary conditions (Torrontegui et al., 2011),

$$
x(t)=d\left[10\left(\frac{t}{t_{f}}\right)^{3}-15\left(\frac{t}{t_{f}}\right)^{4}+6\left(\frac{t}{t_{f}}\right)^{5}\right] .
$$

Once $x(t)$ is defined, Eq. (1) can be easily inverted to give the corresponding expression for the driving term $x_{0}(t)$. Note that there are infinitely many interpolating functions consistent with the boundary conditions at initial and final times. This freedom is quite typical of different STA methods and can be exploited to satisfy other conditions, e.g., minimizing average energy of a particle during displacement. More parameters can be added in the interpolation functions to minimize the quantity of interest (Torrontegui et al., 2011). For instance, the robustness against errors in the value of the angular frequency of the trap $\omega_{0}$ can be enhanced using a Fourier reformulation of the transport problem (Guéry-Odelin and Muga, 2014); see an experimental application by An et al. (2016) and Sec. VII.B.2. The simple example provided here was generalized to take into account anharmonicities (Zhang, Chen, and Guéry-Odelin, 2015) and in 3D to manipulate Bose-Einstein condensates with an atom chip (Corgier et al., 2018).
Section II.H provides a deeper view on the techniques developed to enhance the robustness of a given protocol.

\section{Spin manipulation}

A similar approach can be used for designing the magnetic field components to induce a given trajectory of the mean value of a spin 1/2 $\mathbf{S}(t)$ on the Bloch sphere (Berry, 2009),

$$
\mathbf{B}(t)=B_{0}(t) \mathbf{S}(t)+\frac{1}{\gamma} \mathbf{S}(t) \times \partial_{t} \mathbf{S}(t),
$$

where $\gamma$ is the gyromagnetic ratio and $B_{0}(t)$ is any timedependent function. This solution is found by inverse engineering of the precession equation for the mean value of the spin,

$$
\partial_{t} \mathbf{S}(t)=\gamma \mathbf{B}(t) \times \mathbf{S}(t)
$$

In practice, we usually define the initial and target state and build up the solution by interpolation as before. Such an approach has been used for instance to manipulate a spin by defining the time evolution of the spherical angle of the spin on the Bloch sphere (Vitanov and Shore, 2015; Zhang, Chen, and Guéry-Odelin, 2017). The very same problem can be reformulated using other formalisms such as the Madelung representation (Zhang, Chen, and Guéry-Odelin, 2017) that yields an equation of motion that can be readily reversed. Other formulations of the inverse engineering approach can be made by a proper shaping of the evolution operator (Jing et al., 2013; Kang et al., 2016c) or by time rescaling (Bernardo, 2019). This method has been applied to two- (Zhang, Chen, and Guéry-Odelin, 2017), three- (Kang et al., 2016c; Kang, Huang et al., 2017), and four-level systems (Li, MartínezCercós et al., 2018). Inverse engineering was also used for open quantum systems (Jing et al., 2013; Impens and GuéryOdelin, 2017).

\section{Beyond mean values}

Suppose now that a more detailed specification of the dynamics is needed and we focus on closed, linear quantum systems. We shall design the unitary evolution operator $U(t)$ by specifying a complete basis of dynamical states $\left|\psi_{j}(t)\right\rangle$ assumed to satisfy a time-dependent Schrödinger equation driven by a, to be determined, Hamiltonian,

$$
U(t)=\sum_{j}\left|\psi_{j}(t)\right\rangle\left\langle\psi_{j}(0)\right|
$$

[see other proposals for the form of $U$ by Kang et al., 2016c and Santos, 2018]. The corresponding Hamiltonian is given from the assumed dynamics by

$$
H(t)=i \hbar \dot{U} U^{\dagger}
$$

As in the previous examples, a typical scenario is that the initial and final Hamiltonians are fixed by the experiment or the intended operation. With these boundary conditions the functions $\left|\psi_{j}(t)\right\rangle$ at initial and final times are usually chosen as 
the eigenstates of the corresponding Hamiltonians, but there is freedom to interpolate them, and therefore $H(t)$, in between.

Several approaches depend on different ways to choose the orthogonal basis functions $\left|\psi_{j}(t)\right\rangle$ : (a) in the counterdiabatic driving approach they are instantaneous eigenstates of a reference Hamiltonian $H_{0}(t)$; (b) in invariant-based engineering, they are eigenstates of the invariant of an assumed Hamiltonian form; and (c) more generally they can be just convenient functions. In particular, they can be parametrized so that $H(t)$ obeys certain constraints, such as making zero undesired terms or matrix elements. Note that (a), (b), and (c) are in a certain sense equivalent as they can be reformulated in each other's language, and they all rely on Eq. (6). For example, (b) or (c) do not explicitly need an $H_{0}(t)$, and (a) or (c) do not explicitly need the invariants, but $H_{0}(t)$ or the invariants could be found if needed. In particular, any linear combination $\sum c_{j}\left|\psi_{j}(t)\right\rangle\left\langle\psi_{j}(t)\right|$ with constant coefficients $c_{j}$ is by construction an invariant of motion of $H(t)$. Inverse engineering may also be based on partial information, such as, e.g., using a single function of the set and imposing some additional condition on the Hamiltonian, for example, that the potential is local (i.e., diagonal) and real in coordinate space. These conditions are the essence of the streamlined version (Torrontegui, Martínez-Garaot et al., 2012) of the fast-forward approach (Masuda and Nakamura, 2008, 2010). The following explores all these approaches in more detail.

\section{B. Counterdiabatic driving}

The basic idea of counterdiabatic driving is to add auxiliary interactions to some reference Hamiltonian $H_{0}(t)$ so that the dynamics follows exactly the approximate adiabatic evolution driven by $H_{0}(t)$. An illustrative analogy is a flat, horizontal road turn (the reference) that is modified by inclining the roadway surface about its longitudinal axis with a bank angle so that the vehicles can go faster without sliding off the road. After some precedents (Unanyan et al., 1997; Emmanouilidou et al., 2000), the CD driving paradigm was worked out and developed systematically by Demirplak and Rice (2003, 2005, 2008) for internal state transfer using control fields, then rediscovered in a different but equivalent way as "transitionless tracking" by Berry (2009), and used to design many control schemes after Chen, Lizuain et al. (2010), unaware of the work of Demirplak and Rice, employed Berry's method to control two- and three-level systems.

Berry's formulation. - We start with Berry's formulation because it is somewhat simpler. In Berry (2009), the starting point is a reference Hamiltonian

$$
H_{0}(t)=\sum_{n}|n(t)\rangle E_{n}(t)\langle n(t)|
$$

We adopt for simplicity a notation appropriate for a discrete (real) spectrum and no degeneracies. ${ }^{1} \mathrm{~A}$ state $|n(0)\rangle$ that is

\footnotetext{
${ }^{1}$ Generalizations for degenerate levels, relevant, for example, to speed up holonomic quantum gates, may be found by Takahashi (2013b) and Zhang et al. (2015) or Karzig et al. (2015). Generalizations for non-Hermitian Hamiltonians $H_{0}(t)$ are discussed in Sec. V.D.
}

initially an eigenstate of $H_{0}(0)$ will continue to be so under slow enough driving with the form

$$
\left|\psi_{n}(t)\right\rangle=e^{i \xi_{n}(t)}|n(t)\rangle,
$$

where the adiabatic phases $\xi_{n}(t)$ are found by substituting Eq. (8) as an Ansatz into the time-dependent Schrödinger equation driven by $H_{0}(t)$,

$$
\xi_{n}(t)=-\frac{1}{\hbar} \int_{0}^{t} d t^{\prime} E_{n}\left(t^{\prime}\right)+i \int_{0}^{t} d t^{\prime}\left\langle n\left(t^{\prime}\right) \mid \partial_{t^{\prime}} n\left(t^{\prime}\right)\right\rangle \text {. }
$$

We now seek a Hamiltonian $H(t)$ for which the approximate states $\left|\psi_{n}(t)\right\rangle$ become the exact evolving states,

$$
i \hbar \partial_{t}\left|\psi_{n}(t)\right\rangle=H(t)\left|\psi_{n}(t)\right\rangle
$$

$H(t)$ is constructed using Eq. (6) from the unitary evolution operator

$$
U(t)=\sum_{n} e^{i \xi_{n}(t)}|n(t)\rangle\langle n(0)|
$$

which obeys $i \hbar \partial_{t} U(t)=H(t) U(t)$, so that an arbitrary state evolves as

$$
|\psi(t)\rangle=\sum_{n}|n(t)\rangle e^{i \xi_{n}(t)}\langle n(0) \mid \psi(0)\rangle
$$

After substituting Eq. (11) into Eq. (6), or alternatively differentiating Eq. (12), the Hamiltonian becomes

$$
\begin{gathered}
H(t)=H_{0}(t)+H_{C D}(t), \\
H_{C D}(t)=i \hbar \sum_{n}\left[\left|\partial_{t} n(t)\right\rangle\langle n(t)|\right. \\
\left.-\left\langle n(t) \mid \partial_{t} n(t)\right\rangle|n(t)\rangle\langle n(t)|\right],
\end{gathered}
$$

where $H_{C D}$ is Hermitian and nondiagonal in the $|n(t)\rangle$ basis.

As a simple example of $H_{0}$ and $H_{C D}$, consider a two-level system with reference Hamiltonian

$$
H_{0}(t)=\frac{\hbar}{2}\left(\begin{array}{cc}
-\Delta(t) & \Omega_{R}(t) \\
\Omega_{R}(t) & \Delta(t)
\end{array}\right),
$$

where $\Delta(t)$ is the detuning and $\Omega_{R}(t)$ is the real Rabi frequency. The counterdiabatic Hamiltonian has the form

$$
H_{C D}(t)=\frac{\hbar}{2}\left(\begin{array}{cc}
0 & -i \Omega_{a}(t) \\
i \Omega_{a}(t) & 0
\end{array}\right),
$$

with $\Omega_{a}(t)=\left[\Omega_{R}(t) \dot{\Delta}(t)-\dot{\Omega}_{R}(t) \Delta(t)\right] / \Omega^{2}(t)$ and $\Omega(t)=$ $\sqrt{\Delta^{2}(t)+\Omega_{R}^{2}(t)}$. See further simple examples in Appendix B.

Returning to the general expression (13), we note that $H_{C D}$ is orthogonal to $H_{0}$ considering the scalar product $\operatorname{tr}\left(A^{\dagger} B\right)$ of two operators. Using $d\langle n(t) \mid m(t)\rangle / d t=0$ it can be seen that $H_{C D}$ is also orthogonal to $\dot{H}_{0}$ (Petiziol et al., 2018). An alternative form for $H_{C D}$ is found by differentiating $H_{0}(t)|n(t)\rangle=E_{n}(t)|n(t)\rangle$, 


$$
H_{C D}=i \hbar \sum_{m \neq n} \sum_{n} \frac{|m(t)\rangle\left\langle m(t)\left|\dot{H}_{0}\right| n(t)\right\rangle\langle n(t)|}{E_{n}-E_{m}},
$$

which, using the scaled time $s=t / t_{f}$, gives the scaling $H_{C D} \sim 1 / t_{f}$. In general $H_{C D}(t)$ vanishes for $t<0$ and $t>t_{f}$, either suddenly or continuously at the extreme times, so that the $|n(t)\rangle$ become eigenstates of the full Hamiltonian at the boundary times $t=0^{-}$and $t_{f}^{+}$.

In terms of the phases the total Hamiltonian (13) can be written as (Chen, Torrontegui, and Muga, 2011)

$$
H(t)=J(t)+i \hbar \sum_{n}\left|\partial_{t} n(t)\right\rangle\langle n(t)|
$$

with

$$
J(t)=-\hbar \sum_{n}|n(t)\rangle \dot{\xi}_{n}(t)\langle n(t)|
$$

Subtracting $H-H_{C D}$ we get an alternative form of $H_{0}$ consistent with Eqs. (7) and (9),

$$
H_{0}=\hbar \sum_{n}|n(t)\rangle\left[-\dot{\xi}_{n}+i\langle n(t) \mid \dot{n}(t)\rangle\right]\langle n(t)| .
$$

If we write the same $H_{0}(t)$ [Eq. (7)] as before in an alternative basis with different phases $H_{0}(t)=\sum_{n}\left|n^{\prime}(t)\right\rangle E_{n}(t)\left\langle n^{\prime}(t)\right|$, where $\left|n^{\prime}(t)\right\rangle=e^{i \phi_{n}}|n(t)\rangle$, the formalism goes through using primed functions $\xi_{n}^{\prime}$ and corresponding primed operators. [One example is $\left|n^{\prime}(t)\right\rangle=e^{\xi_{n}(t)}|n(t)\rangle$ so that $\xi_{n}^{\prime}(t)=0$.] It is easy to check though that the terms compensate so that $H_{C D}^{\prime}(t)=H_{C D}(t)$, i.e., a change of representation for $H_{0}(t)$ does not change $H(t)$, nor the CD-driving term, nor the physics.

Quite a different issue is to change the physics when imposing a set of basis functions $\{|n(t)\rangle\}$ and a set of phases $\left\{\xi_{n}(t)\right\}$ which are not a priori regarded as adiabatic (Chen, Torrontegui, and Muga, 2011). This procedure is essentially invariant-based inverse engineering since one is imposing some specific dynamics (thus some invariants) without presupposing a given $H_{0}(t) . U(t)$ in Eq. (11) becomes the primary object and the driving Hamiltonian is given by Eq. (18) with diagonal part (20), which defines $H_{0}(t)$, and a coupling, nondiagonal part (14). Now, changing the phases $\xi_{n}(t)$ modifies $H_{0}(t)$ and has an impact on the physical evolution of a general wave function $|\psi(t)\rangle$. Of course the populations $\quad \mathcal{P}_{n}(t) \equiv|\langle n(t) \mid \psi(t)\rangle|^{2}=\mathcal{P}_{n}(0) \quad$ driven by Eq. (18) are not affected by phase shifts. The $\dot{\xi}_{n}(t)$ can be optimized to minimize energy costs (Hu et al., 2018), robustness against decoherence (Santos and Sarandy, 2018), or intensity of the extra CD controls (Santos et al., 2019).

A related procedure is to set the $|n(t)\rangle$ but consider $H_{0}(t)$ and the eigenvalues $E_{n}(t)$ controllable elements rather than given (Berry, 2009). For example, setting all $E_{n}(t)=0$ cancels the dynamical phase. That means that for a given set of states $|n(t)\rangle, H_{C D}(t)$ alone (without an $H_{0}$ ) drives the same populations than $H(t)=H_{0}(t)+H_{C D}(t)$ for any choice of $E_{n}(t)$ (Chen, Lizuain et al., 2010).

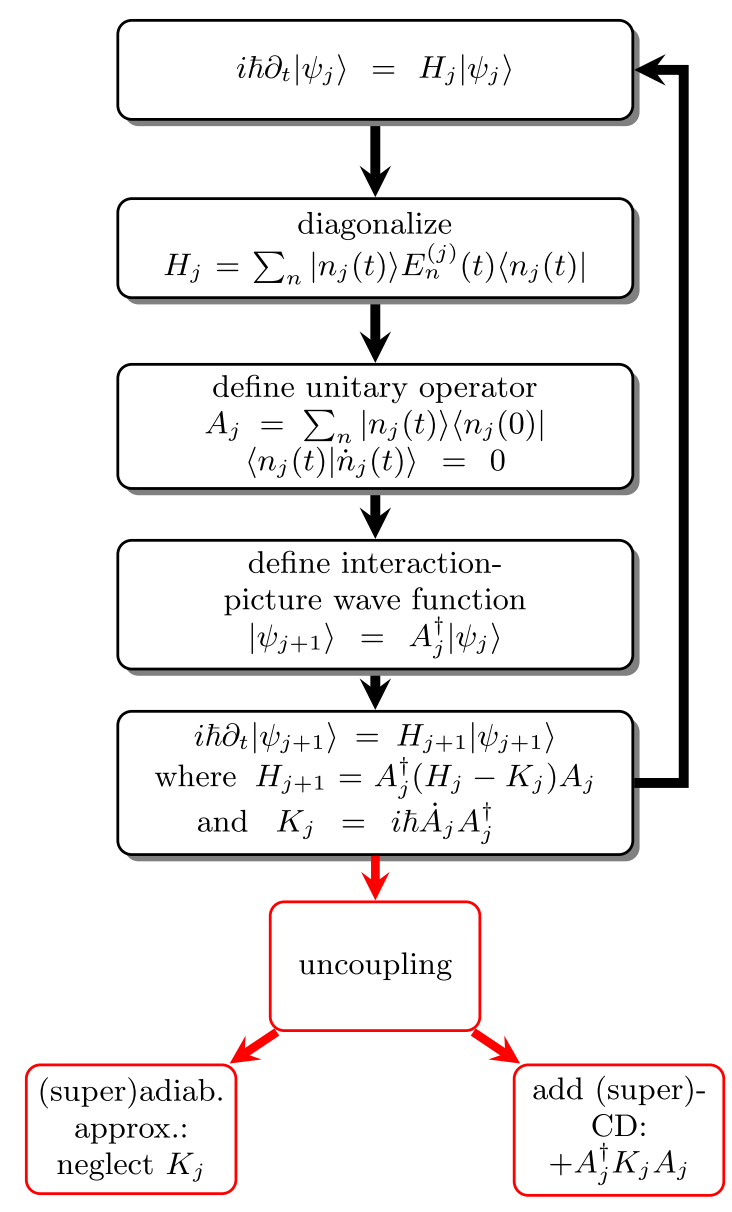

FIG. 3. Scheme for superadiabatic iterative interaction pictures. At the end of each iteration it is possible, apart from starting a new one, to either neglect the nondiagonal coupling in $H_{j+1}$ (adiabatic approximation if $j=0$ or superadiabatic approximation for $j \geq 1$ ) or add a term (CD for $j=0$ or super-CD otherwise) to the Hamiltonian $H_{j+1}$ to exactly cancel the coupling.

The formulation of Demirplak and Rice.-To follow some past and recent developments and generalizations it is worth finding the auxiliary Hamiltonian $H_{C D}$ by the equivalent formulation of Demirplak and Rice (2003, 2005, 2008), which is the zeroth iteration $j=0$ in Fig. 3 .

In the following discussion we concentrate on $j=0$. Among the possible phase choices for the eigenstates of $H_{0}(t)$, we now use the one that satisfies the "parallel transport" condition $\left\langle n_{0}(t) \mid \dot{n}_{0}(0)\right\rangle=0$. Regardless of the "working" basis of eigenvectors $|n(t)\rangle$ one starts with, the parallel transported basis is found as

$$
\left|n_{0}(t)\right\rangle=e^{-\int_{0}^{t}\left\langle n\left(t^{\prime}\right) \mid \dot{n}\right\rangle d t^{\prime}}|n(t)\rangle
$$

Later on we shall see the consequences of applying or not applying parallel transport. A dynamical solution driven by $H_{0}(t)$ is written as $\left|\psi_{0}(t)\right\rangle$ and the instantaneous eigenvalues as $E_{n}^{(0)}(t)$. The extra notational burden of the index $j=0$ may be ignored in many applications, in particular, for ordinary $C D$ 
driving, but it will be useful to cope with higher order suparadiabatic iterations later on.

The way to find the auxiliary driving term is to first express the dynamics in an adiabatic frame. For that we apply the transformation $\left|\psi_{1}(t)\right\rangle=A_{0}^{\dagger}(t)\left|\psi_{0}(t)\right\rangle$ to the dynamical states driven by $H_{0}(t)$, with $A_{0}(t)=\sum_{n}\left|n_{0}(t)\right\rangle\left\langle n_{0}(0)\right|$. In the resulting adiabatic frame the coupling, diabatic terms are made obvious, $-A_{0}^{\dagger} K_{0} A_{0}$, where $K_{0}=i \hbar \dot{A}_{0} A_{0}^{\dagger}=$ $\sum_{n}\left|\dot{n}_{0}(t)\right\rangle\left\langle n_{0}(t)\right|$. In this adiabatic frame we may (a) neglect the coupling (adiabatic approximation), or (b) cancel the coupling by adding $A_{0}^{\dagger} K_{0} A$ to the Hamiltonian; see again Fig. 3. In the original, Schrödinger picture, also referred to as the laboratory frame hereafter, this addition amounts to using $H=H_{0}+K_{0}$.

An alternative useful form for $K_{0}(t)$ is (Messiah, 1962)

$$
K_{0}(t)=i \hbar \sum_{n} \frac{d P_{n}(t)}{d t} P_{n}(t),
$$

where $\quad P_{n}(t)=\left|n_{0}(t)\right\rangle\left\langle n_{0}(t)|=| n(t)\right\rangle\langle n(t)| . \quad K_{0} \quad$ may as well be written in an arbitrary basis of eigenvectors $|n(t)\rangle$, i.e., not necessarily parallel transported, $K_{0}(t)=$ $i \hbar \sum_{n}|\dot{n}(t)\rangle\langle n(t)|-| n(t)\rangle\langle n(t) \mid \dot{n}\rangle\langle n(t)|=H_{C D}(t)$. This result proves that $K_{0}$ is identical to $H_{C D}$ in Eq. (14). Since the projectors $P_{n}(t)=\left|n_{0}(t)\right\rangle\left\langle n_{0}(t)|=| n(t)\right\rangle\langle n(t)|$ are invariant with respect to phase choices of the eigenvectors, the form in which $H_{C D}$ is usually written in Eq. (14) is invariant under different choices of phases for the eigenstates. In particular, this implies that $K_{0}$ is purely nondiagonal [Kato's condition (Kato, 1950; Demirplak and Rice, 2008)] in the arbitrarily chosen basis of eigenvectors of $H_{0}(t),|n(t)\rangle$.

If we now return to the parallel transported basis in the interaction (adiabatic) picture, the addition of $A_{0}^{\dagger} K_{0} A_{0}$ cancels the couplings so the dynamics is trivially solved with dynamical phase factors. In the Schrödinger picture,

$$
\left|\psi_{0}(t)\right\rangle=e^{-(i / \hbar) \int_{0}^{t} E_{n}\left(t^{\prime}\right) d t^{\prime}}\left|n_{0}(t)\right\rangle\left\langle n_{0}(0) \mid \psi_{0}(0)\right\rangle,
$$

which is exactly Eq. (12) as can be seen by using Eq. (21).

If, instead of $A_{0}$, a more general transformation $\tilde{A}=$ $\sum_{n}|n(t)\rangle\langle n(0)|$ is used, the coupling term in the interaction picture becomes $-\tilde{A}_{0}^{\dagger} \tilde{K}_{0} \tilde{A}_{0}$, where

$$
\begin{aligned}
\tilde{K}_{0} & =i \hbar \dot{\tilde{A}}_{0} \tilde{A}_{0}^{\dagger} \\
& =\sum_{n}|\dot{n}(t)\rangle\left\langle n(t)\left|=K_{0}+i \hbar\right| n(t)\right\rangle\langle n(t) \mid \dot{n}(t)\rangle\langle n(t)|
\end{aligned}
$$

and its cancellation would lead in the laboratory picture to a different state,

$$
\sum_{n} e^{-(i / \hbar) \int_{0}^{t} E_{n}\left(t^{\prime}\right) d t^{\prime}}|n(t)\rangle\left\langle n(0) \mid \psi_{0}(0)\right\rangle,
$$

although the probabilities are not affected.

Demirplak and Rice (2008) also pointed out that the operator

$$
H_{C D}^{[n]}=i \hbar\left[d P_{n} / d t, P_{n}\right]_{-},
$$

which fulfils $P_{m} H_{C D} P_{n}=P_{m} H_{C D}^{[n]} P_{n}$ and $P_{n} H_{C D} P_{m}=$ $P_{n} H_{C D}^{[n]} P_{m}$ for all $m$, as well as $P_{m} H_{C D}^{[n]} P_{m}^{\prime}=0$ for $m$, $m^{\prime} \neq n$, uncouples the dynamics of level $n$. For $H_{0}+H_{C D}^{[n]}$, $e^{i \xi_{n}(t)}|n(t)\rangle$ is an exact solution of the dynamics. This is an interesting simplification as we are often interested in only one state, typically the ground state. The last condition imposed by Demirplak and Rice (2008), $P_{m} H_{C D}^{[n]} P_{m}^{\prime}=0$ for $m, m^{\prime} \neq n$, is not really necessary for the uncoupling of the $n$th level. Without it, a broad set of "state-dependent" CD operators $H_{C D}^{[n]}+Q_{n} B Q_{n}$, where $Q_{n}=1-P_{n}$ and $B$ is any Hermitian operator, can be generated. This multiplicity may be useful and explains why different auxiliary state-dependent CD terms have been proposed (Patra and Jarzynski, 2017b; Setiawan et al., 2017).

\section{Superadiabatic iterations}

Let us recap before moving ahead. The adiabatic interaction picture (IP) corresponds to expressing the quantum dynamics in the adiabatic basis of instantaneous eigenstates of $H_{0}(t)$. The dynamical equation in the adiabatic IP includes an effective Hamiltonian $H_{1}(t)$ with a diagonal (adiabatic) term and a coupling term; see Fig. 3 .

We can repeat the sequence iteratively. In the first "superadiabatic" iteration, $H_{1}$ is diagonalized to find its instantaneous (parallel-transported) eigenstates $\left|n_{1}(t)\right\rangle$. With the new "superadiabatic basis" a new IP is generated driven by an effective Hamiltonian $\mathrm{H}_{2}$ with a diagonal part in the superadiabatic basis and a coupling term. This new coupling term $-A_{1}^{\dagger} K_{1} A_{1}$ may be (a) neglected (superadiabatic approximation) or (b) canceled by adding its negative, and so on. The canceling term to be added to $H_{0}$ in the Schrödinger picture is $H_{c d}^{(j)}=B_{j} K_{j} B_{j}^{\dagger}$, with $B_{0}=1$ and $B_{j}=\prod_{k=0}^{j-1} A_{k}$ for $j \geq 1$. Note that in general only the one generated in the zeroth iteration agrees with the standard CD term $H_{c d}^{0}=H_{C D}$.

The recursive iterations were worked out by Garrido (1964), without considering the cancellations, to find out generalizations of the adiabatic approximation. Berry (1987) also used them to calculate a sequence of corrections to Berry's phase and introduced the name "superadiabatic transformations." Demirplak and Rice (2008) proposed to apply the superadiabatic iterative frame to generate alternative (to the simple CD approach) higher order coupling-canceling terms. Later Ibáñez, Chen, and Muga (2013) made explicit the conditions that the derivatives of $H_{0}(t)$ must satisfy at the time boundaries in order to really generate a shortcut to adiabaticity (rather than just a shortcut to superadiabaticity), i.e., a protocol that takes instantaneous eigenstates of $H_{0}(t=0)$ to corresponding eigenstates of $H_{0}\left(t_{f}\right)$.

The naive expectation that each iteration will produce smaller and smaller couplings does not hold in general. They decrease up to an optimal iteration and then grow (Berry, 1987). Working with the optimal frame may or may not be worthwhile depending on whether the boundary conditions for derivatives of $H_{0}(t)$ are fulfilled (Ibáñez, Chen, and Muga, 2013). 
An interesting feature of the superadiabatic sequence of coupling terms is that their operator structure changes with the iteration. For example, for a two-level system with Hamiltonian $X \sigma_{x}+Z(t) \sigma_{z}, X$ is constant, where the $\sigma_{x, y, z}$ are Pauli matrices, the first (adiabatic) CD term $K_{0}$ is of the form $Y(t) \sigma_{y}$, see Eq. (16), whereas the second (first-order superadiabatic) coupling term in the Schrödinger frame reproduces the structure of $H_{0}$ with $x$ and $z$ components but not a $y$ component. [For three-level systems see Huang et al. (2016), Kang et al. (2016b), Song, Ai et al. (2016), and $\mathrm{Wu}, \mathrm{Su}, \mathrm{Ji}$, and Zhang (2017).] Unitarily transforming $K_{0}$ (see Sec. II.B.2) also provides a Hamiltonian without a $y$ component, which is different from the term $H_{c d}^{(1)}=$ $A_{0} K_{1} A_{0}^{\dagger}$ generated from the first superadiabatic iteration, see an explicit comparison by Ibáñez et al. (2012), where $H_{c d}^{(1)}$ was shown to have smaller intensity than $H_{C D}$.

To avoid confusion we discourage the use of the expression superadiabatic to refer to the regular CD approach (in fact a zeroth order in the superadiabatic iterative frame) or its unitarily transformed versions discussed in Sec. II.B.2. This use of the word superadiabatic, as being equivalent to $\mathrm{CD}$ driving or even generically to all shortcuts is, however, somewhat extended.

DRAG controls. - The DRAG framework was developed to avoid diabatic transitions to undesired levels in the context of superconducting quantum devices. It was recently reviewed by Theis et al. (2018) but we sketch the main ideas here since it is related to the superadiabatic scheme as formulated, e.g., by Ibáñez, Chen, and Muga (2013). A typical scenario is that the (super)-CD term $H_{c d}^{(j)}=B_{j} K_{j} B_{j}^{\dagger}$ is not physically feasible and does not match the controls in the lab. The DRAG approach addresses this problem by decomposing the controllable Hamiltonian that "corrects" the dynamics as $H^{\text {ctrl }}=$ $\sum_{k} u_{k}(t) h_{k}+$ H.c. with control fields $u_{k}(t)$ and coupling terms $h_{k}$. In a given superadiabatic frame partial contributions to $u_{k}(t)$ are found by projecting $H_{c d}^{(j)}$ into the assumed Hamiltonian structure. This generates an approximation to the exact uncoupling term so the diabatic coupling, even if not canceled exactly, is reduced. An important point is that further iterations, in contrast with the bare superadiabatic iterations, typically converge, so that the coupling eventually vanishes. A variant of this approach applies when the error terms are not independently controlled, because they all depend on some common control, e.g., a single laser field. Different perturbative approximations using a power series in the inverse gap energies were worked out systematically (Theis et al., 2018).

\section{Beyond the basic formalism}

The CD Hamiltonian $H_{C D}$ often implies different operators from those in $H_{0}$ that typically may be hard or even impossible to generate in the laboratory. Moreover, a lot of spectral information is in principle used to build $H_{C D}$, specifically the eigenvectors of $H_{0}$, so a number of strategies, reviewed hereafter, are put forward to avoid some terms in the auxiliary Hamiltonian, and/or the spectral information needed. Changing the phases $\xi_{n}(t)$ without modifying the $|n(t)\rangle$ changes $H_{0}$ but not $H_{C D}$, so it is not enough for these purposes (Ibáñez, Martínez-Garaot et al., 2011).

\section{a. "Physical" unitary transformations}

A useful method to generate alternative, physically feasible shortcuts from an existing shortcut generated by counterdiabatic driving or otherwise, is to perform physical, rather than formal, unitary transformations (Ibáñez et al., 2012), or corresponding canonical transformations in classical systems (Deffner, Jarzynski, and del Campo, 2014). Given a Hamiltonian $H(t)$ that drives the wave function $|\psi(t)\rangle$, the unitarily transformed state $\left|\psi^{\prime}(t)\right\rangle=\mathcal{U}^{\dagger}(t)|\psi(t)\rangle$ is driven by the Hamiltonian (the primes here distinguish the picture, they do not represent derivatives)

$$
\begin{gathered}
H^{\prime}(t)=\mathcal{U}^{\dagger}(H-K) \mathcal{U}, \\
K=i \hbar \dot{\mathcal{U}} \mathcal{U}^{\dagger} .
\end{gathered}
$$

If we set $\mathcal{U}(0)=\mathcal{U}\left(t_{f}\right)=1$, then the wave functions coincide at the boundary times $|\psi(0)\rangle=\left|\psi^{\prime}(0)\right\rangle$ and $\left|\psi\left(t_{f}\right)\right\rangle=$ $\left|\psi^{\prime}\left(t_{f}\right)\right\rangle$. If, in addition, $\dot{\mathcal{U}}(0)=\dot{\mathcal{U}}\left(t_{f}\right)=0$, then also the Hamiltonians coincide at boundary times $H(0)=H^{\prime}(0)$ and $H\left(t_{f}\right)=H^{\prime}\left(t_{f}\right)$.

Note that here the alternative Hamiltonian form (26) and the state $\left|\psi^{\prime}(t)\right\rangle$ are not just convenient mathematical transforms of $H(t)$ or $|\psi(t)\rangle$ representing the same physics as in conventional interaction or Heisenberg picture transformations. Instead, at intermediary times, $H^{\prime}(t)$ and $H(t)$ represent indeed different (laboratory) drivings, and $|\psi(t)\rangle$ and $\left|\psi^{\prime}(t)\right\rangle$ different dynamical states. This was emphasized by S. Ibáñez et al. (2012) by calling the different alternatives "multiple Schrödinger pictures."

The art is to find a useful $\mathcal{U}(t)$ to make $H^{\prime}(t)$ feasible. This approach was applied in many works; see, e.g., Hollenberg (2012), Takahashi (2015), Agundez et al. (2017), and Sels and Polkovnikov (2017), and several experiments (Bason et al., 2012; Zhang et al., 2013; An et al., 2016; Du et al., 2016). Deffner, Jarzynski, and del Campo (2014) applied it to generate feasible (i.e., involving local potentials in coordinate space, independent of momentum) Hamiltonians for scaleinvariant dynamical processes.

When $H(t)$ is a linear combination of generators $G_{a}$ of some Lie algebra,

$$
\left[G_{b}, G_{c}\right]=\sum_{a=1}^{N} \alpha_{a b c} G_{a}
$$

where the $\alpha_{a b c}$ are the structure constants, $\mathcal{U}$ may be constructed by exponentiating elements of the algebra and imposing the vanishing of the unwanted terms (MartínezGaraot et al., 2014). To carry out the transformation an element $G$ of the Lie algebra of the Hamiltonian is chosen,

$$
\mathcal{U}(t)=e^{-i g(t) G},
$$

where $g(t)$ is a real function to be set. This type of unitary operator $\mathcal{U}(t)$ constitutes a "Lie transform." Note that $K$ in Eq. (27) becomes $-\hbar \dot{g}(t) G$ and commutes with $G$ so $H^{\prime}$ is given by 


$$
\begin{aligned}
\mathcal{U}^{\dagger}(H-K) \mathcal{U}= & e^{i g G}(H-K) e^{-i g G} \\
= & H-\hbar \dot{g} G+i g[G, H]-\frac{g^{2}}{2 !}[G,[G, H]] \\
& -i \frac{g^{3}}{3 !}[G,[G,[G, H]]]+\cdots,
\end{aligned}
$$

which depends only on $G, H$, and its nested commutators with $G$, so it stays in the algebra. If we can choose $G$ and $g(t)$ so that the undesired generator components in $H(t)$ cancel out and the boundary conditions for $\mathcal{U}$ are satisfied, the method provides a feasible, alternative shortcut. A simple example for the two-level Hamiltonian is given in Appendix A. MartínezGaraot et al. (2014) and Kang et al. (2018) provided more examples.

Interaction pictures.-Frequently the $\mathrm{CD}$ terms are found formally in a transformed picture, $I$ in Fig. 4, which is intended only as a mathematical aid. The effective Hamiltonian $H_{I}$ in this picture, without the CD term, represents the same physics as some original Hamiltonian $H_{S}$ in the Schrödinger picture $S$ in Fig. 4. Once the CD term is added we get a new Hamiltonian $H_{I}^{\prime}$ that when transformed back gives $H_{S^{\prime}}$. It happens often that simple-looking auxiliary terms in the transformed frames become difficult to implement in the laboratory frame. In Sec. III.G we comment on some $N$ body models with easily solvable CD terms in a convenient transformed frame but hard to realize in the lab frame. Alternative unitary transformations $U_{\tilde{\varphi}}$, different from the one used to go between the $I$ and $S$ pictures $U_{\varphi}$, may help to solve the problem, giving from $H_{I^{\prime}}$ feasible shortcuts driven by a new lab frame Hamiltonian $H_{S^{\prime \prime}}$. Ibáñez et al. (2015) worked out this alternative route for two-level systems when the rotating wave approximation is not applicable; see also Ibáñez, Peralta Conde et al. (2011), Chen and Wei (2015), and H. Li et al. (2017). Physics beyond the rotating wave approximation is of much current interest due to the increasing use of strong fields and microwave frequencies, for example, in $\mathrm{N} V$ centers.

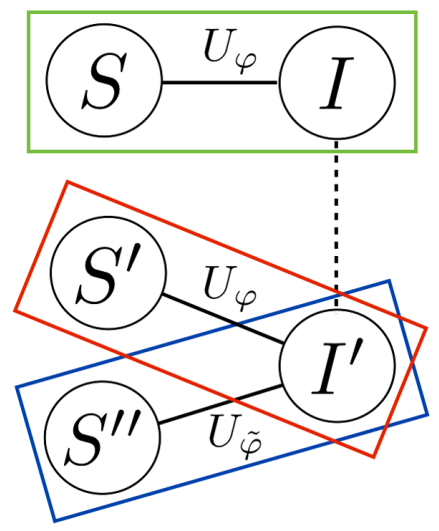

FIG. 4. Schematic relation between different Schrödinger and interaction pictures. Each node corresponds also to different Hamiltonians. The rectangular boxes enclose nodes that represent the same underlying physics. The solid lines represent unitary relations for the linked states and the dashed line represents a nonunitary addition of an auxiliary term to the Hamiltonian.

\section{$b$. Schemes that focus on one state}

A simplifying assumption that helps one to find simpler decoupling terms is to focus on only one state, typically the ground state $|0(t)\rangle$ or a subset of states. Explicit exact forms of the driving that uncouples that state were worked out quite early (Demirplak and Rice, 2008), see Eq. (25), and more recently for systems described in coordinate space in Patra and Jarzynski (2017b) or in a discrete basis (Setiawan et al., 2017). As for approximate schemes, Opatrný and Mølmer (2014) proposed to add to $H_{0}(t)$ the Hamiltonian $H_{B}(t)=\sum_{k}^{K} f_{k}(t) T_{k}$ using only feasible interactions (i.e., available experimentally) $T_{k}$, where the $f_{k}(t)$ are amplitudes found by minimizing the norm of $\left(H_{B}-H_{C D}\right)|0(t)\rangle$. A related approach uses the Lyapunov control theory (Ran et al., 2017). Similarly, Chen, Xia et al. (2016) and Chen et al. (2017) achieved feasible auxiliary Hamiltonians by adding adjustable Hamiltonians that nullify unwanted nonadiabatic couplings for specific transitions.

\section{c. Effective counterdiabatic field}

In Petiziol et al. $(2018,2019) H_{0}(t)=\sum_{k} u_{k}(t) H_{k}$ is written as before in terms of control functions $u_{k}(t)$ and available time-independent control Hamiltonians $H_{k}$. Using control theory arguments it is found that $H_{C D}$ necessarily belongs to the corresponding dynamical Lie algebra, i.e., the smallest algebra that contains the $-i H_{k}$ and the nested commutators. As well, the action of $H_{C D}$ can be approximated by using only the initially available Hamiltonians in an "effective counterdiabatic" Hamiltonian $H_{E}=\sum e_{k}(t) H_{k}$. To implement $H_{E}$ in Petiziol et al. $(2018,2019)$, the control functions $e_{k}(t)$ are chosen as periodic functions of period $T$ with the form of a truncated Fourier expansion $\sum_{k} \mathcal{A}_{k} \sin (k \omega t)+\mathcal{B}_{k} \cos (k \omega t)$, where $\omega=2 \pi / T$.

The coefficients are determined by setting the first terms of the Magnus expansion generated by $H_{E}$ to match those of the desired evolution stroboscopically at multiples of $T$ and interpolating smoothly in between. Avoided crossing problems and entanglement creation are addressed with this technique. Note alternative uses of the Magnus expansion in the WAHWAH technique of Schutjens et al. (2013), aimed at producing fast pulses to operate on a qubit without interfering with other qubits in frequency-crowded systems, and in Claeys et al. (2019).

\section{d. Dressed-states approach}

CD driving is generalized by Baksic, Ribeiro, and Clerk (2016) by considering a dressed-states approach which uses three different dynamical pictures. In this summary the notation and even the terminology differs from Baksic, Ribeiro, and Clerk (2016); see Table I.

First consider a Schrödinger picture description where the driving Hamiltonian is $H_{0}(t)+H_{c}(t)$ with reference Hamiltonian $H_{0}(t)$ as in Eq. (7), and driven wave function $|\psi(t)\rangle . H_{c}(t)$ will be found later so that the total Hamiltonian satisfies some conditions. Then a first rotating picture defined by $\left|\psi_{I}(t)\right\rangle=U(t)^{\dagger}|\psi(t)\rangle$ is introduced, where $U(t)=$ $\sum_{n}|n(t)\rangle\langle n|$ and the $|n\rangle$ are time independent. A simple choice is $|n\rangle=|n(0)\rangle$ that insures $U(0)=U\left(t_{f}\right)=1$. Since $|n(t)\rangle$ are instantaneous (adiabatic) eigenstates of $H_{0}(t)$ 
D. Guéry-Odelin et al.: Shortcuts to adiabaticity: Concepts, methods, ...

TABLE I. Scheme for "dressed-state" driving (Baksic, Ribeiro, and Clerk, 2016).

\begin{tabular}{lccc}
\hline \hline Picture & Wave function & Unitary transformation & Hamiltonian \\
\hline Schrödinger & $\psi(t)$ & $H=H_{0}+H_{c}$ \\
First rotating picture ("adiabatic") & $\psi_{I}(t)=U^{\dagger} \psi(t)$ & $U=\sum_{n}|n(t)\rangle\langle n|$ & $H_{I}=i \hbar \dot{U}^{\dagger} U+U^{\dagger} H U$ \\
Second rotating picture ("dressed") & $\psi_{I I}(t)=V^{\dagger} \psi_{I}(t)$ & $V=\sum_{n}|\tilde{n}(t)\rangle\langle n|$ & $H_{I I}=i \hbar \dot{V}^{\dagger} V+V^{\dagger} H_{I} V$ \\
\hline \hline
\end{tabular}

we may naturally call this picture the "adiabatic frame" with driving Hamiltonian $H_{I}(t)$; see Table I. Then a second rotating frame is introduced as $\left|\psi_{I I}(t)\right\rangle=V(t)^{\dagger}\left|\psi_{I}(t)\right\rangle$, with $V(t)=$ $\sum_{n}|\tilde{n}(t)\rangle\langle n|$, driven by $H_{I I}(t) .\{|\tilde{n}(t)\rangle\}$ are called dressed states and it is assumed that $V(0)=V\left(t_{f}\right)=1$. The method to generate shortcuts is to choose $V(t)$, i.e., the functions $|\tilde{n}(t)\rangle$ and $H_{c}$ so that $H_{I I}(t)$ is diagonal in the basis $\{|n\rangle\}$, $H_{I I}(t)=\sum_{n}|n\rangle E_{n}^{I I}(t)\langle n|$. Back to the Schrödinger picture this means that no transitions occur among states $U(t) V(t)|n\rangle=U(t)|\tilde{n}(t)\rangle$.

$V(t)$ is chosen to ensure that $H_{c}(t)$ is feasible. The two nested transformations make the method more involved and less intuitive than standard CD driving. To gain insight note that if $V(t)=1$ for all $t$ the method reduces to standard CD driving. One can think of $V$ as a way to add flexibility to the inverse engineering so as to drive states in the Schrödinger picture along uncoupled $U(t) V(t)|n\rangle$ vectors rather than along vectors $U(t)|n\rangle=|n(t)\rangle$. The latter are given, up to phases, once $H_{0}(t)$ is specified, whereas $U(t) V(t)|n\rangle$ may still be manipulated to find a convenient $H_{c}(t)$ and possibly minimize the occupancy of some state to be avoided, e.g., because of spontaneous decay (Baksic, Ribeiro, and Clerk, 2016).

For applications see Baksic et al. (2017), Coto et al. (2017), Liu et al. (2017), Wu, Ji, and Zhang (2017b), B. B. Zhou et al. (2017), and X. Zhou et al. (2017).

\section{e. Variational approach}

Motivated by difficulties to diagonalize $H_{0}(t)$ and the nonlocalities in the exact counterdiabatic Hamiltonian in many-body systems, Kolodrubetz et al. (2017) and Sels and Polkovnikov (2017) developed a variational method to construct approximate counterdiabatic Hamiltonians without using spectral information. The starting point in Sels and Polkovnikov (2017) is a unitary transformation $U[\lambda(t)]=$ $\sum_{n}|n[\lambda(t)]\rangle\langle n|$ to rotate the state $|\psi(t)\rangle$ that evolves under a time-dependent Hamiltonian $H_{0}[\lambda(t)]$, to the moving frame state $|\tilde{\psi}(t)\rangle=U^{\dagger}[\lambda(t)]|\psi(t)\rangle$, which satisfies the effective Schrödinger equation

$$
i \hbar \partial_{t}|\tilde{\psi}\rangle=\left\{\tilde{H}_{0}[\lambda(t)]-\dot{\lambda} \tilde{A}_{\lambda}\right\}|\tilde{\psi}\rangle,
$$

where $\tilde{H}_{0}$ is diagonal in the $|n\rangle$ basis and $\tilde{A}_{\lambda}$ is the adiabatic "gauge potential" in the moving frame,

$$
\begin{aligned}
\tilde{H}_{0}[\lambda(t)] & =U^{\dagger} H_{0}[\lambda(t)] U=\sum_{n} E_{n}(\lambda)|n\rangle\langle n|, \\
\tilde{A}_{\lambda} & =i \hbar U^{\dagger} \partial \lambda U .
\end{aligned}
$$

All nonadiabatic transitions are produced by the gauge potential. In the counterdiabatic approach the system is driven by the Hamiltonian

$$
H(t)=H_{0}+\dot{\lambda} A_{\lambda},
$$

where $A_{\lambda}=U \tilde{A}_{\lambda} U^{\dagger}=i \hbar\left(\partial_{\lambda} U\right) U^{\dagger}$ such that in the moving frame $H_{0}^{\text {eff }}=\tilde{H}_{0}$ is diagonal and no transitions are allowed. Up to now we have only introduced a new notation and terminology in Eqs. (31)-(33). ${ }^{2}$ In the adiabatic limit $|\dot{\lambda}| \rightarrow 0$ the Hamiltonian (33) reduces to the original one $H(t)=H_{0}(t)$. After differentiating the first equation in Eq. (32), the gauge potential satisfies (Jarzynski, 2013)

$$
i \hbar\left(\partial_{\lambda} H_{0}+F_{a d}\right)=\left[A_{\lambda}, H_{0}\right]
$$

where $F_{a d}=-\sum_{n} \partial_{\lambda} E_{n}(\lambda)|n(\lambda)\rangle\langle n(\lambda)|$ is the adiabatic force operator. Since, by construction, $F_{a d}$ commutes with $H_{0}$, Eq. (34) implies that

$$
\left[i \hbar \partial_{\lambda} H_{0}-\left[A_{\lambda}, H_{0}\right], H_{0}\right]=0
$$

where the difficult-to-calculate force has been eliminated. This equation can be used to find the adiabatic gauge potentials directly without diagonalizing the Hamiltonian. Moreover, solving this equation is analogous to minimizing the HilbertSchmidt norm of the operator

$$
G_{\lambda}=\partial_{\lambda} H_{0}+\frac{i}{\hbar}\left[A_{\lambda}^{*}, H_{0}\right]
$$

with respect to $A_{\lambda}^{*}$, where $A_{\lambda}^{*}$ is a trial gauge potential. For an application to prepare ground states in a lattice gauge model, see Hartmann and Lechner (2018).

Potential problems with this scheme are that it may be difficult to know what local operators should be included in the variational basis, and moreover their practical realizability is not guaranteed. To solve these two difficulties, Claeys et al. (2019) expanded the gauge potential in terms of nested commutators of the Hamiltonian $H_{0}$ and the driving term $\partial_{\lambda} H_{0}$, even if these do not close a Lie algebra. Since the commutators also arise in the Magnus expansion in Floquet systems, they can be realized up to arbitrary order using Floquet engineering, i.e., periodical driving (Boyers et al., 2018). The expansion coefficients can be calculated either analytically or variationally. The method can be adapted to suppress excitations in a known frequency window. Application examples were provided for a two- and a three-level system, where one, respectively, two terms

\footnotetext{
${ }^{2}$ We assumed for simplicity a dependence on time via a single parameter $\lambda$. A more general dependence on a vector $\lambda$ is worked out by Deffner, Jarzynski, and del Campo (2014) and Nishimura and Takahashi (2018) which leads to a decomposition of the CD term and a "zero curvature condition" among the CD components.
} 
returned the exact gauge potential, and to a many-body spin chain, where a limited number of terms and an approximate gauge potential resulted in a drastic increase in fidelity. Further useful properties of the expansion are that it relates the locality of the gauge potential to the order in the expansion, and that it remains well defined in the thermodynamic and classical limits.

\section{f. Counterdiabatic Born-Oppenheimer dynamics}

Duncan and del Campo (2018) proposed to exploit the separation of fast and slow variables using the BornOppenheimer approximation. Simpler CD terms (compared to the exact $\mathrm{CD}$ term) for the fast and slow variables can be found in two steps avoiding the diagonalization of the full Hamiltonian. The method is tested for two coupled harmonic oscillators and a system of two charged particles.

\section{g. Constant CD-term approximation}

Oh and Kais (2014) proposed, in the context of the adiabatic Grover's search algorithm implemented by a two-level system, to substitute the exact CD term by a constant term. This method improves the scaling of the nonadiabatic transitions with respect to the running time. J. Zhang et al. (2018) performed an experiment with a single trapped ion choosing the constant term with the aid of a numerical simulation.

In a different vein Santos and Sarandy (2018) discussed the conditions for $H_{0}(t)$ and phase choice necessary in the unitary evolution operator (11) to implement an exact constant driving.

\section{Invariants and scaling laws}

Dynamical invariants and invariant-based engineering constitute a major route to design STA protocols. The basic reason, already sketched in Eqs. (5) and (6), is that, in linear systems, determining the desired dynamics amounts to setting dynamical invariants of motion, and the Hamiltonian may in principle be found from them. This connection is quite general and is also valid classically (Lewis and Leach, 1982), but it is mostly applied for systems in which the Hamiltonian form and corresponding invariants are known explicitly as functions of auxiliary parameters that satisfy auxiliary equations consistent with the dynamical equation and the invariant.

\section{Lewis-Riesenfeld invariants}

Originally proposed in 1969, a Lewis-Riesenfeld invariant (Lewis and Riesenfeld, 1969) for a Hamiltonian $H(t)$ is a Hermitian operator $I(t)$ which satisfies

$$
\frac{d I}{d t}=\frac{\partial I}{\partial t}+\frac{i}{\hbar}[H, I]=0
$$

so that the expectation values for states driven by $H(t)$ are constant in time. Since $I(t)$ is a constant of motion it has time-independent eigenvalues. If $\left|\phi_{n}(t)\right\rangle$ is an instantaneous eigenstate of $I(t)$, a solution of the Schrödinger equation $i \hbar \partial_{t}\left|\psi_{n}(t)\right\rangle=H(t)\left|\psi_{n}(t)\right\rangle$ can be constructed as $\left|\psi_{n}(t)\right\rangle=e^{i \alpha_{n}(t)}\left|\phi_{n}(t)\right\rangle$. Here $\alpha_{n}(t)=(1 / \hbar) \int_{0}^{t}\left\langle\phi_{n}(s)\right|\left[i \hbar \partial_{s}-\right.$ $H(s)]\left|\phi_{n}(s)\right\rangle d s$ is the Lewis-Riesenfeld phase. Hence a general solution to the Schrödinger equation can be written as

$$
|\psi(t)\rangle=\sum_{n} c_{n}\left|\psi_{n}(t)\right\rangle
$$

where the $c_{n}$ are independent of time.

These invariants were originally used to solve for the state driven by a known time-dependent Hamiltonian (Lewis and Riesenfeld, 1969). In shortcuts to adiabaticity this idea is reversed (Chen, Ruschhaupt et al., 2010) and the Hamiltonian is found from a prescribed state evolution. Formally, a time evolution operator of the form

$$
U=\sum_{n} e^{i \alpha_{n}(t)}\left|\phi_{n}(t)\right\rangle\left\langle\phi_{n}(0)\right|
$$

implies a Hamiltonian $H(t)=i \hbar \dot{U} U^{\dagger}$. This is the essence of invariant-based inverse engineering.

For a given Hamiltonian there are many possible invariants. For example, the density operator describing the evolution of a system is a dynamical invariant. The choice of which particular invariant to use is made on the basis of mathematical convenience. Invariants have also been generalized to nonHermitian invariants and Hamiltonians (Gao, Xu, and Qian, 1992; Lohe, 2009; Ibáñez, Martínez-Garaot et al., 2011), as well as open systems (see Sec. V).

A connection to adiabaticity is asymptotic. In the limit of long operation times (i.e., adiabatic), Eq. (37) becomes $[H, I] \approx 0$ and the dynamics prescribed by invariant-based engineering will approach adiabatic dynamics driven by $H$. Hence for long times, $H(t)$ and $I(t)$ have approximately a common eigenbasis for all times.

Other relations to adiabaticity do not need long times nor common bases for $H(t)$ and $I(t)$ at all times. In particular, demanding only that the invariant and the Hamiltonian commute at the start and the end of the process, i.e., $[I(0), H(0)]=\left[I\left(t_{f}\right), H\left(t_{f}\right)\right]=0$, the eigenstates of the invariant and the Hamiltonian coincide at initial and final times, but may differ with each other at intermediate times because the commutativity is not imposed. If no level crossings take place, the final state will keep the initial populations for each $n$th level, as in an adiabatic process, but in a finite (short, faster-than-adiabatic) time. This leaves freedom to choose how the state evolves in the intermediate time and then use Eq. (37) to find the Hamiltonian that drives such a state evolution. The flexibility can be exploited to improve the stability of the schemes against noise and systematic errors; see Sec. II.H.

The relation of the invariant-based approach to $\mathrm{CD}$ driving (Chen, Torrontegui, and Muga, 2011) implies a different connection to adiabaticity. So far we have not mentioned in this section any "reference" $H_{0}(t)$, but by reinterpreting $\left|\phi_{n}(t)\right\rangle$ as eigenvectors of $H_{0}(t)$, and the Lewis-Riesenfeld phases as adiabatic phases, an implicit $H_{0}(t)$ operator may be written down using Eq. (20). Hence an implicit $H_{C D}(t)$ follows from subtraction, $H_{C D}(t)=H(t)-H_{0}(t)$. In invariantbased engineering, however, these implicit operators are not really used and do not play any role in practice. As a consequence of the different emphases and construction 
recipes for $H(t)$, given some initial and final Hamiltonians, STA protocols designed via $\mathrm{CD}$ or invariant approaches are often very different.

Lie algebras.-As first noted by Sarandy, Duzzioni, and Serra (2011), invariant-based inverse engineering can also be formulated in terms of dynamical Lie algebras. The assumption of a Lie structure is also used to classify and construct dynamical invariants for four-level or smaller nontrivial systems for specific applications (Güngördü et al., 2012; Herrera et al., 2014; Kiely et al., 2016). The approaches by Martínez-Garaot et al. (2014) and Petiziol et al. (2018), already discussed, also make use of the Lie algebraic structure.

Torrontegui, Martínez-Garaot, and Muga (2014) provided a bottom-up construction procedure of the Hamiltonian using invariants and Lie algebras. Let us assume that the Hamiltonian of a system $H(t)$ and the invariant $I(t)$ can be written as linear combinations of Hermitian operators $G_{a}$ (generators),

$$
H(t)=\sum_{a=1}^{N} h_{a}(t) G_{a}, \quad I(t)=\sum_{a=1}^{N} f_{a}(t) G_{a},
$$

that form a Lie algebra closed under commutation; see Eq. (28). Inserting these forms into Eq. (37), we get that

$$
\dot{f}_{a}(t)-\sum_{b=1}^{N} \mathcal{A}_{a b}(t) h_{b}(t)=0
$$

where the $N \times N$ matrix $\mathcal{A}$ is defined by

$$
\mathcal{A}_{a b} \equiv \frac{1}{i \hbar} \sum_{c=1}^{N} \alpha_{a b c} f_{c}(t)
$$

and the $\alpha_{a b c}$ are the structure constants. In vector form,

$$
\partial_{t} \vec{f}(t)=\mathcal{A} \vec{h}(t)
$$

where the vectors $\vec{f}(t)$ and $\vec{h}(t)$ represent the invariant and Hamiltonian, respectively. The inversion trick is to first choose the auxiliary functions $\vec{f}(t)$ (and therefore the state evolution) and infer $\vec{h}(t)$ from this. Technically the inversion requires introducing a projector $\mathcal{Q}$ for the null subspace of $\mathcal{A}$ and the complementary projector $\mathcal{P}$. In $\mathcal{P}$ subspace a pseudoinverse matrix can be defined, and the $\mathcal{Q}$ component of $\vec{h}$ is chosen to make the resulting Hamiltonian realizable (Torrontegui, Martínez-Garaot, and Muga, 2014). Examples for two[SU(2) algebra] and three-level systems (with the fourdimensional algebra U3S3) were provided. Levy et al. (2018) reformulated this formalism in terms of the density operator and used it to design robust control protocols against the influence of different types of noise. In particular, they developed a method to construct a control protocol which is robust against dissipation of the population and minimizes the effect of dephasing.

\section{Examples of invariant-based inverse engineering}

Lewis-Riesenfeld invariants have been used to design state transfer schemes in two-level (Ruschhaupt et al., 2012; Martínez-Garaot et al., 2013; Kiely and Ruschhaupt, 2014), three-level (Chen and Muga, 2012a; Kiely and Ruschhaupt, 2014; Benseny et al., 2017), and four-level systems (Güngördü et al., 2012; Herrera et al., 2014; Kiely et al., 2016), or Hamiltonians quadratic in creation and annihilation operators (Stefanatos and Paspalakis, 2018a). They are also useful to design motional dynamics in harmonic traps or otherwise. Many more applications specific for different system types may be found in Sec. III. Here we provide two basic examples, the two-level model and the Lewis-Leach family of potentials for a particle of mass $m$ moving in 1D.

\section{a. Two-level system}

We now present an example of a two-level system with a Hamiltonian given by

$$
H(t)=\frac{\hbar}{2}\left(\begin{array}{cc}
-\Delta(t) & \Omega_{R}(t)-i \Omega_{I}(t) \\
\Omega_{R}(t)+i \Omega_{I}(t) & \Delta(t)
\end{array}\right),
$$

and an invariant of the form

$$
I(t)=\frac{\hbar}{2}\left(\begin{array}{cc}
\cos [\theta(t)] & \sin [\theta(t)] e^{-i \alpha(t)} \\
\sin [\theta(t)] e^{i \alpha(t)} & -\cos [\theta(t)]
\end{array}\right) .
$$

From the equation that defines the invariant, Eq. (37), $\theta(t)$ and $\alpha(t)$ must satisfy

$$
\begin{gathered}
\dot{\theta}=\Omega_{I} \cos \alpha-\Omega_{R} \sin \alpha, \\
\dot{\alpha}=-\Delta-\cot \theta\left(\Omega_{R} \cos \alpha+\Omega_{I} \sin \alpha\right) .
\end{gathered}
$$

The eigenvectors of $I(t)$ are

$$
\begin{aligned}
& \left|\phi_{+}(t)\right\rangle=\left(\begin{array}{c}
\cos (\theta / 2) e^{-i \alpha / 2} \\
\sin (\theta / 2) e^{i \alpha / 2}
\end{array}\right), \\
& \left|\phi_{-}(t)\right\rangle=\left(\begin{array}{c}
\sin (\theta / 2) e^{-i \alpha / 2} \\
-\cos (\theta / 2) e^{i \alpha / 2}
\end{array}\right),
\end{aligned}
$$

with eigenvalues $\pm \hbar / 2$. The angles $\alpha$ and $\theta$ can be thought of as spherical coordinates on the Bloch sphere. The general solution of the Schrödinger equation is then a linear combination of the eigenvectors of $I(t)$, i.e., $|\Psi(t)\rangle=$ $c_{+} e^{i \kappa_{+}(t)}\left|\phi_{+}(t)\right\rangle+c_{-} e^{i \kappa_{-}(t)}\left|\phi_{-}(t)\right\rangle$, where $c_{ \pm} \in \mathbb{C}$ and

$$
\dot{\kappa}_{ \pm}(t)=\frac{1}{\hbar}\left\langle\phi_{ \pm}(t)\left|\left[i \hbar \partial_{t}-H(t)\right]\right| \phi_{ \pm}(t)\right\rangle .
$$

Therefore, it is possible to construct a particular solution

$$
|\psi(t)\rangle=\left|\phi_{+}(t)\right\rangle e^{-i \gamma(t) / 2},
$$

where $\gamma= \pm 2 \kappa_{ \pm}$and 
D. Guéry-Odelin et al:: Shortcuts to adiabaticity: Concepts, methods, ...

$$
\dot{\gamma}=\frac{1}{\sin \theta}\left(\Omega_{R} \cos \alpha+\Omega_{I} \sin \alpha\right)
$$

Using Eqs. (46), (47), and (51) we can retrieve the physical quantities in terms of the auxiliary functions,

$$
\begin{gathered}
\Omega_{R}=\cos \alpha \sin \theta \dot{\gamma}-\sin \alpha \dot{\theta}, \\
\Omega_{I}=\sin \alpha \sin \theta \dot{\gamma}+\cos \alpha \dot{\theta}, \\
\Delta=-\cos \theta \dot{\gamma}-\dot{\alpha} .
\end{gathered}
$$

If the functions $\alpha, \gamma$, and $\theta$ are chosen with the appropriate boundary conditions, different state manipulations are possible. For example, $\theta(0)=0$ and $\theta\left(t_{f}\right)=\pi$ imply perfect population inversion at a time $t_{f}$. Note the freedom to interpolate along different paths.

\section{b. Lewis-Leach family}

Consider a one-dimensional Hamiltonian $H=p^{2} / 2 m+$ $V(q, t)$ with potential (Lewis and Leach, 1982)

$$
\begin{aligned}
V(q, t)= & -F(t) q+\frac{m}{2} \omega^{2}(t) q^{2} \\
& +\frac{1}{\rho(t)^{2}} U\left[\frac{q-q_{c}(t)}{\rho(t)}\right]+g(t) .
\end{aligned}
$$

These Hamiltonians have a quadratic in momentum invariant

$$
\begin{aligned}
I= & \frac{1}{2 m}\left[\rho\left(p-m \dot{q_{c}}\right)-m \dot{\rho}\left(q-q_{c}\right)\right]^{2} \\
& +\frac{1}{2} m \omega_{0}^{2}\left(\frac{q-q_{c}}{\rho}\right)^{2}+U\left(\frac{q-q_{c}}{\rho}\right),
\end{aligned}
$$

provided the functions $\rho, q_{c}, \omega$, and $F$ satisfy the auxiliary equations

$$
\begin{aligned}
\ddot{\rho}+\omega^{2}(t) \rho & =\frac{\omega_{0}^{2}}{\rho^{3}}, \\
\ddot{q_{c}}+\omega^{2}(t) q_{c} & =F(t) / m,
\end{aligned}
$$

with $\omega_{0}$ a constant. The first equation is known as the Ermakov equation (Ermakov, 1880), while the second is the Newton equation of motion for a forced harmonic oscillator. They can be found by inserting the quadratic-in$p$ invariant, Eq. (56), into Eq. (37). The properties of such invariants have also been formulated in terms of Feynman propagators (Dhara and Lawande, 1984).

For this family of Hamiltonians we can explicitly calculate the Lewis-Riesenfeld phase,

$$
\alpha_{n}(t)=-\frac{1}{\hbar} \int_{0}^{t} d t^{\prime}\left[\frac{\lambda_{n}}{\rho^{2}}+\frac{m\left[\left(\dot{q}_{c} \rho-q_{c} \dot{\rho}\right)^{2}-\omega_{0}^{2} q_{c}^{2} / \rho^{2}\right]}{2 \rho^{2}}+g\right] \text {, }
$$

and the eigenvectors in coordinate representation,

$$
\begin{aligned}
& \phi_{n}(q, t) \\
& \quad=\exp \left\{\frac{i m}{\hbar}\left[\dot{\rho} q^{2} / 2 \rho+\left(\dot{q}_{c} \rho-q_{c} \dot{\rho}\right) q / \rho\right]\right\} \rho^{-1 / 2} \Phi_{n}\left(\frac{q-q_{c}}{\rho}\right),
\end{aligned}
$$

where $\Phi_{n}(\sigma)$ is a solution of the stationary Schrödinger equation

$$
\left[-\frac{\hbar^{2}}{2 m} \frac{\partial^{2}}{\partial \sigma^{2}}+\frac{1}{2} m \omega_{0}^{2} \sigma^{2}+U(\sigma)\right] \Phi_{n}=\lambda_{n} \Phi_{n},
$$

with $\sigma=\left(q-q_{c}\right) / \rho$. This quadratic invariant has been instrumental in designing many of the schemes which manipulate trapping potentials for expansions, compressions, transport, launching, stopping, or combined processes. It is key, for example, to manipulate the motion of trapped ions, see Sec. III.A, and in proposals to implement STA-based interferometry (Dupont-Nivet, Westbrook, and Schwartz, 2016; Martínez-Garaot, Rodriguez-Prieto, and Muga, 2018).

Designing first the function $\rho(t)$ which determines the wave function width, and $q_{c}(t)$ (a classical particle trajectory), the force $F(t)$ and $\omega(t)$ can be determined using Eq. (57). The boundary values of the auxiliary functions at the time limits are fixed to satisfy physical conditions and commutativity of $H$ and the invariant. The nonunique interpolation is usually done with polynomials or trigonometric functions.

Expansions.-Invariant-based inverse engineering for Lewis-Leach Hamiltonians was first implemented by Chen, Ruschhaupt et al. (2010) to cool down a trapped atom by expanding the trap. Expansions using invariant-based engineering were first implemented with ultracold atoms in a pioneering experiment on STA techniques by Schaff et al. (2010) and Schaff, Capuzzi et al. (2011). Such cooling protocols have also been envisioned to optimize sympathetic cooling (Choi, Onofrio, and Sundaram, 2011; Onofrio, 2016). For very short processes, $t_{f}<1 /\left(2 \omega_{f}\right)$, where $\omega_{f}$ is the trap frequency at the final time, $\omega^{2}(t)$ may become negative during some time interval. While this implies a transient repulsive potential, the atoms always remain confined (Chen, Ruschhaupt et al., 2010). A repulsive potential may or may not be difficult to implement depending on the physical setting. For example, the analysis by Torrontegui et al. (2018) suggested that it is viable for trapped ions.

Compared to the simplicity of invariant-based engineering, the $\mathrm{CD}$ approach for expansions or compressions provides, for an $H_{0}$ characterized by some predetermined time-dependent frequency $\omega(t)$, a nonlocal, cumbersome counterdiabatic term $H_{C D}=-(p q+q p) \dot{\omega} /(4 \omega)$ (Muga et al., 2010). However, a unitary transformation produces a new shortcut with local potential and modified frequency (Ibáñez et al., 2012)

$$
\omega^{\prime}=\left[\omega^{2}-\frac{3 \dot{\omega}^{2}}{4 \omega^{2}}+\frac{\ddot{\omega}}{2 \omega}\right]^{1 / 2}
$$

see further connections among the two Hamiltonians in del Campo (2013) and Mishima and Izumida (2017).

So far we have considered only one-dimensional motion. Formally, the three coordinates in an ideal harmonic trap are 
uncoupled so expansion or transport processes can be treated independently. However, in many cold atom experiments changing the intensity of a laser beam simultaneously affects the longitudinal and transversal frequencies (Torrontegui, Chen, Modugno, Ruschhaput et al., 2012).

Transport.-Designing particle transport has been another major application of the invariant (56) (Torrontegui et al., 2011; Ness et al., 2018; Tobalina, Alonso, and Muga, 2018); see also Secs. II.G and III.A. Note that $U$ in Eq. (55) is arbitrary. Setting $\omega=\omega_{0}=0$, and $\rho=1, F=m \ddot{q}_{c}$ plays the role of a "compensating force" that cancels the inertial effects of a moving $U\left[q-q_{c}(t)\right]$ so that the wave function stays at rest in the frame moving with $q_{c}{ }^{3} q_{c}(t)$ can be chosen as an arbitrary function connecting the desired initial and final trap positions. The same solution is reached applying fast forward (Masuda and Nakamura, 2010), or using unitary transformations combined with the CD approach (Ibáñez et al., 2012), which in principle provides the difficult-to-realize term $H_{C D}=p \dot{q}_{c} \cdot{ }^{4}$

Deffner, Jarzynski, and del Campo (2014) discussed more generally that for Hamiltonians of the form

$$
H_{0}=\frac{p^{2}}{2 m}+\frac{1}{\gamma^{2}} U\left(\frac{q-f}{\gamma}\right)
$$

the (nonlocal) $\mathrm{CD}$ term is

$$
H_{C D}=\frac{\dot{\gamma}}{2 \gamma}[(q-f) p+p(q-f)]+\dot{f} p,
$$

and found the generic unitary transformation that provides the local auxiliary terms that appear in Eq. (55). More general cases for multiparticle systems are discussed in the following section.

Tobalina et al. (2017) used the invariants to design shortcuts to adiabaticity for nonrigid driven transport and to launch particles in harmonic and general potentials. Compared to rigid transport, nonrigid transport requires a more demanding manipulation, but it also provides a wider range of control opportunities, for example, to achieve narrow velocity distributions in a launching process, suitable for accurate ion implantation or low-energy scattering experiments.

\section{Scaling laws}

For many-body systems constructing the Lewis-Riesenfeld invariant is in general much more involved. Takahashi (2017b)

\footnotetext{
${ }^{3}$ For rigidly moving harmonic traps, a formal alternative to the compensating force is to set $U=0$ and $F=m \omega_{0}^{2} q_{0}(t)$, keeping $\omega(t)=\omega_{0}$ as the trap frequency (Torrontegui et al., 2011). The two routes may be shown to be equivalent up to a gauge time-dependent term; see, e.g., Tobalina, Alonso, and Muga (2018).

${ }^{4}$ See, however, An et al. (2016) and Sec. III.E for a possible realization. Note that $\mathrm{CD}$ terms like $-(p q+q p) \dot{\omega} /(4 \omega)$ or $p \dot{q}_{c}$ anticommute with the time reversal operator $\Theta$ (Sels and Polkovnikov, 2017). Microscopic irreversibility holds by changing the sign of the external forces in the backward trajectories (Campisi, Hänggi, and Talkner, 2011). In the two examples above this means to change $\dot{\omega} \rightarrow-\dot{\omega}$ and $\dot{q}_{c} \rightarrow-\dot{q}_{c}$.
}

avoided this difficulty for an infinite-range Ising model in a transverse field by constructing an invariant using a meanfield Ansatz. However, another approach is to exploit scaling laws. If the Hamiltonian fulfills certain scaling laws one can determine the invariant in a similar manner as in Sec. II.C.2.b. Specifically the Hamiltonian

$$
\begin{aligned}
H(t)= & \sum_{i=1}^{N}\left\{\frac{\mathbf{p}_{i}^{2}}{2 m}+U\left[\mathbf{q}_{i}, \lambda(t)\right]\right\}+\epsilon(t) \sum_{i<j} V\left(\mathbf{q}_{i}-\mathbf{q}_{j}\right) \\
& +\sum_{i=1}^{N}\left[-\frac{m}{2} \frac{\ddot{\gamma}}{\gamma}\left(\mathbf{q}_{i}-\mathbf{f}\right)^{2}-m \ddot{\mathbf{f}} \cdot \mathbf{q}_{i}\right],
\end{aligned}
$$

describing $N$ interacting particles with the following scaling laws (Deffner, Jarzynski, and del Campo, 2014):

$$
\begin{aligned}
U[\mathbf{q}, \lambda(\mathbf{t})] & =U_{0}\{[\mathbf{q}-\mathbf{f}(t)] / \gamma(t)\} / \gamma(t)^{2}, \\
V(\kappa \mathbf{q}) & =\kappa^{-\alpha} V(\mathbf{q}), \\
\epsilon(t) & =\gamma(t)^{\alpha-2},
\end{aligned}
$$

where $U_{0}(\mathbf{q})=U[\mathbf{q}, \lambda(0)]$, has the invariant

$$
\begin{aligned}
I= & \sum_{i=1}^{N} \frac{1}{2 m}\left[\gamma\left(\mathbf{p}_{i}-m \dot{\mathbf{f}}\right)-m \dot{\gamma}\left(\mathbf{q}_{i}-\mathbf{f}\right)\right]^{2} \\
& +\sum_{i=1}^{N} U_{0}\left(\frac{\mathbf{q}_{i}-\mathbf{f}}{\gamma}\right)+\sum_{i<j} V\left(\frac{\mathbf{q}_{i}-\mathbf{q}_{j}}{\gamma}\right) .
\end{aligned}
$$

The method based on Lewis-Riesenfeld invariants is not applicable to nonlinear equations such as the Gross-Pitaevskii equation, but the presence of scaling laws can still prove beneficial for inverse engineering. Scaling solutions for the Gross-Pitaevskii equation were first noticed by Castin and Dum (1996) and Kagan, Surkov, and Shlyapnikov (1996) and were exploited to perform STA expansions in harmonic traps by Muga et al. (2009). For example, the 1D equation

$$
\begin{aligned}
& i \hbar \partial_{t} \psi(x, t) \\
& \quad=\left[-\frac{\hbar^{2}}{2 m} \frac{\partial^{2}}{\partial x^{2}}+\frac{1}{2} m \omega^{2}(t) x^{2}+g(t)|\psi(x, t)|^{2}\right] \psi(x, t),
\end{aligned}
$$

with $g(t)=g_{0} / \rho(t)$ has scaling solutions of the form

$$
\psi(x, t)=\rho^{-1 / 2} e^{i m \dot{\rho} x^{2} / 2 \hbar \rho} e^{-i \mu \tau(t) / \hbar} \Psi(x / \rho, 0)
$$

provided that the following consistency equations, including the Ermakov equation, are fulfilled:

$$
\begin{aligned}
\ddot{\rho}+\omega(t)^{2} \rho & =\frac{\omega_{0}^{2}}{\rho^{3}}, \\
\tau(t) & =\int_{0}^{t} \frac{d t^{\prime}}{\rho^{2}\left(t^{\prime}\right)},
\end{aligned}
$$

and $\Psi(y, \tau)$ satisfies 


$$
i \hbar \frac{\partial \Psi}{\partial \tau}=-\frac{\hbar^{2}}{2 m} \frac{\partial^{2} \Psi}{\partial y^{2}}+\frac{m \omega_{0}^{2}}{2} y^{2} \Psi+g_{0}|\Psi|^{2} \Psi
$$

Different dimensions imply different scalings. In 1D and 3D traps, the STA scaling solutions for the frequency change are found for either a simultaneous change of the time dependence of the coupling or a Thomas-Fermi type of regime, whereas 2D traps are privileged in this respect since none of these conditions are needed.

These methods were realized experimentally for trapped Bose-Einstein condensates (Schaff, Song et al., 2011; Rohringer et al., 2015). Scaling solutions also exist for a class of many-body systems including interacting quasi-1D Bose gases (Gritsev, Barmettler, and Demler, 2010) and were applied to construct shortcuts for a square box (del Campo and Boshier, 2012), and other trapping potentials (del Campo, 2013; Deffner, Jarzynski, and del Campo, 2014).

Inverse engineering the transport of condensates was worked out by Torrontegui, Chen, Modugno, Schmidt et al. (2012) using as an Ansatz the scaling provided by invariant theory for linear dynamics. Scaling has also been used to design STA protocols for the fast expansion of a condensate in an optical lattice (Yuce, 2012), possibly pumped from a reservoir (Ozcakmakli and Yuce, 2012).

Fermi gas.-Papoular and Stringari (2015) put to work a new class of exact scaling solutions for 3D "unitary," Fermi gases and 2D weakly interacting Bose gases in anisotropic time-dependent harmonic traps with initial and final traps having the same frequency ratios. These solutions may be useful to implement a microscope for quantum defects hosted by the cloud, avoiding the strong distortion due to free expansion. The proposal of Papoular and Stringari (2015) was realized experimentally by Deng, Chenu et al. (2018) and Deng, Diao et al. (2018) for a 3D Fermi gas "at unitarity."

\section{Connection with Lax pairs}

Lax pairs were originally introduced by Peter Lax in 1968 (Lax, 1968). A completely integrable nonlinear partial differential equation (PDE) can be associated with a Lax pair and Lax pairs have been used to find the solution $u(x, t)$ of the corresponding nonlinear PDE. The key objective was to construct a pair of linear differential operators $L=L(u)$, $M=M(u)$ in such a way that the equation

$$
\partial_{t} L(u)+[L(u), M(u)]=0
$$

is fulfilled if and only if $u$ is a solution of the initial nonlinear PDE. Let $\psi(t, x)$ be the eigenvectors of $L(u)$,

$$
L(u) \psi=\lambda \psi,
$$

where it follows from Eq. (71) that the eigenvalues $\lambda$ must be time independent. It also follows from Eq. (71) that

$$
\partial_{t} \psi=M(u) \psi
$$

This transforms the problem of solving the nonlinear PDE for $u(x, t)$ to that of solving the linear equation (71) [respectively, the linear equations (72) and (73)] which is often easier to solve than the initial nonlinear PDE.

The relation between Lax pairs and shortcuts with a counterdiabatic Hamiltonian was first noted by Okuyama and Takahashi (2016). They considered systems for which $H_{0}$ is the invariant for $H=H_{0}+H_{C D}$ (see Sec. II.B). From the equation defining the invariant the counterdiabatic Hamiltonian can be determined by

$$
\partial_{t} H_{0}=\frac{i}{\hbar}\left[H_{0}, H_{C D}\right]
$$

By comparing Eqs. (71) and (74), the connection with Lax pairs is given by setting $L=H_{0}$ and $M=-(i / \hbar) H_{C D}$. Okuyama and Takahashi (2016) first considered $H_{0}=p^{2}+$ $u(x, t)$ and $H_{C D}$ containing third order in $p$ terms. This results in the nonlinear Korteweg-de Vries equation for the physical potential $u(x, t)$. The advantage is that a complete set of solutions for this nonlinear equation can now be found. Based on these solutions, the exact counterdiabatic term for a particle in a hyperbolic Scarf potential was determined, and unitary transformations were applied to generate feasible auxiliary Hamiltonians avoiding the cubic-in- $p$ term. They discussed a spin lattice as a second example. When considering instead that the invariant takes the form $\gamma^{2}(t) H_{0}$ and $H_{C D}$ includes up to first orders in $p$, as in Eq. (63), the scale-invariant potential (62) follows. It is also possible to extend the approach to non-scale-invariant systems.

A different but similar route is to regard Lewis-Riesenfeld invariants (Lewis and Riesenfeld, 1969) and Lax pairs (the invariants were proposed $1 \mathrm{yr}$ after the Lax pairs) as two sides of the same coin (Kiely and Ruschhaupt, 2019), relating Eqs. (37) and (74) by the alternative connection $L=I$ and $M=-(i / \hbar) H$. This approach facilitates extending the domain of invariant-based shortcuts, for example, using cubic-in- $p$ invariants with feasible interactions.

\section{Variational methods}

We have already discussed an STA variational approach in Sec. II.B.2.e (Kolodrubetz et al., 2017; Sels and Polkovnikov, 2017) for CD driving. Here we review other variational proposals.

Takahashi (2013a, 2015) reformulated invariant-based engineering or counterdiabatic approaches in terms of a quantum brachistochrone variational problem, with the action

$$
S=\int_{0}^{T} d t\left(\mathcal{L}_{T}+\mathcal{L}_{S}+\mathcal{L}_{C}\right)
$$

and Lagrangians corresponding to the constraints for the process time $\left(\mathcal{L}_{T}\right)$, the Schrödinger equation $\left(\mathcal{L}_{S}\right)$, and additional experimental constraints $\left(\mathcal{L}_{C}\right)$. This formulation is used to examine the stability of the driving, noting that processes are stable against variations in operators which commute with $H_{C D}$ and unstable against variations in those that anticommute with it. This work has been extended to classical, stochastic finite-sized systems described by a continuous-time master equation to find an optimal transition-rate matrix (Takahashi and Ohzeki, 2016). 
A different variational approach was worked out by Li, Sun, and Chen (2016) and J. Li et al. (2018). These works considered a BEC trapped in a harmonic trap. Quantum dynamical equations follow by minimizing the action defined from a Lagrangian density, using some Ansatz for the wave function. This allows one to cope with systems that would otherwise be difficult to treat (e.g., with no scaling laws available). However, the quality of the approximate dynamics strongly depends on the Ansatz chosen.

Li, Sun, and Chen (2016) used this approach to control matter waves in harmonic traps. Using as an Ansatz a bright solitary wave solution, approximate auxiliary equations analogous to Eqs. (57) are found. The Newton equation for the wave center remains the same, whereas the Ermakov equation is modified to include a term that depends on the nonlinear coupling parameter. Using only a time-dependent control of this parameter via a Feschbach resonance, the soliton wave function can be compressed or expanded in nonadiabatic time scales with high fidelity.

Applying the same concepts, an efficient quantum heat engine running an Otto cycle with a condensate as its working medium was proposed by J. Li et al. (2018). The engine strokes are done on a short timescale ensuring a large power output and high efficiency. Also, Fogarty et al. (2019) applied the variational approach to design the time-dependent interaction strength between two ultracold atoms so as to create entangled states.

\section{E. Fast forward}

The so-called FF approach was first derived to accelerate a given quantum dynamics by mimicking the effect of an FF button in an audio or video player (Masuda and Nakamura, 2008). The original idea was to use a scaling transformation $\tau=\alpha t$, for some constant $\alpha$ (inhomogeneous scalings are also possible) so that the solutions $\psi_{0}(\tau)$ of $i \hbar \partial_{\tau} \psi_{0}(\tau)=$ $H(\tau) \psi_{0}(\tau)$ are just scaled in time as $\psi_{0}(\tau)=\psi_{\alpha}(t)$ with respect to the solutions of $i \hbar \partial_{t} \psi_{\alpha}(t)=\alpha H(\alpha t) \psi_{\alpha}(t)$. This concept works formally and in some discrete Hamiltonians it is experimentally viable. However, if $H$ includes a kinetic energy it implies that the mass should be changed (Bernardo, 2019). Masuda and Nakamura (2008) solved this problem by modifying the potential but not the mass.

\section{The original formalism}

Consider the time-dependent Schrödinger equation

$$
i \hbar \frac{\partial \psi_{0}(\mathbf{r}, t)}{\partial t}=-\frac{\hbar^{2}}{2 m} \Delta \psi_{0}(\mathbf{r}, t)+V_{0}(\mathbf{r}, t) \psi_{0}(\mathbf{r}, t)
$$

in a time interval $[0, T]$ with real potential $V_{0}$. To speed up the dynamics keeping the potential real, a nontrivial phase factor that depends on the coordinates has to be added to the FF wave function,

$$
\psi_{F F}(\mathbf{r}, t)=\psi_{0}(\mathbf{r}, \Lambda(t)) e^{i \varphi(\mathbf{r}, t)},
$$

driven by the FF potential

$$
\begin{aligned}
V_{F F}(\mathbf{r}, t)= & -\hbar \ddot{\Lambda} \Sigma(\mathbf{r}, \Lambda(t))-2 \hbar[\dot{\Lambda}-1] \partial_{t} \Sigma(\mathbf{r}, \Lambda(t)) \\
& -\frac{\hbar^{2}}{2 m}\left(\dot{\Lambda}^{2}-1\right)\left[\partial_{\mathbf{r}} \Sigma(\mathbf{r}, \Lambda(t))\right]^{2}+V_{0}(\mathbf{r}, \Lambda(t))
\end{aligned}
$$

The phase is given by $\varphi(\mathbf{r}, t)=[\dot{\Lambda}-1] \Sigma(\mathbf{r}, \Lambda(t))$, where $\Sigma$ is the phase of the reference wave function $\psi_{0}(\mathbf{r}, t)=\tilde{\psi}_{0}(\mathbf{r}, t) \exp [i \Sigma(\mathbf{r}, t)]$. Imposing $\Lambda(0)=0, \Lambda\left(t_{f}\right)=T$, $\dot{\Lambda}(0)=\dot{\Lambda}\left(t_{f}\right)=1$, and $\ddot{\Lambda}(0)=\ddot{\Lambda}\left(t_{f}\right)=0$ implies that $V_{F F}(\mathbf{r}, 0)=V_{0}(\mathbf{r}, 0)$ and $V_{F F}\left(\mathbf{r}, t_{f}\right)=V_{0}(\mathbf{r}, T)$. Moreover, the additional phase $\varphi(\mathbf{r}, t)$ vanishes at the boundary of the time interval $\left[0, t_{f}\right]$. For $\dot{\Lambda}>1$, the dynamics is accelerated while it is slowed down for $0<\dot{\Lambda}<1$. A negative $\dot{\Lambda}$ corresponds to a time reversed evolution.

The transposition of these results to speed up an adiabatic evolution is not direct because the adiabatic evolution holds only for an infinitely slow process that needs infinite magnification factors. A first but too naive approach consists of considering the adiabatic wave function as a candidate for applying the previous fast-forward formalism. If the system is in the $n$th eigenstate $\phi_{n}(\mathbf{r}, \lambda)$ associated with the eigenvalue $E_{n}(\lambda)$, where $\lambda$ accounts for the parameters, the corresponding adiabatic wave function reads

$$
\psi_{\mathrm{ad}}(\mathbf{r}, t, \lambda(t))=\phi_{n}(\mathbf{r}, \lambda(t)) e^{-i \varphi_{\mathrm{dyn}}(t)+i \varphi_{\mathrm{ad}}(t)},
$$

where $\varphi_{\text {dyn }}(t)=\int_{0}^{t} E_{n}\left(\lambda\left(t^{\prime}\right)\right) d t^{\prime} / \hbar$ is the dynamical phase. The direct route fails and a renormalization is needed modifying both the wave function and the Hamiltonian in a consistent manner to ensure that the wave function remains valid for a finite change of the parameters (Masuda and Nakamura, 2010).

To highlight the smallness of the change of parameter, Masuda and Nakamura (2010) introduced the constant rate $\varepsilon \ll 1$ associated with $\lambda \rightarrow \lambda+\delta \lambda$ where $\delta \lambda=\varepsilon t$. A finite change of the parameter $\lambda$ during the time interval $\left[0, t_{f}\right]$ is given by

$$
\lambda\left(\Lambda\left(t_{f}\right)\right)-\lambda(0)=\varepsilon \Lambda\left(t_{f}\right)
$$

The expression for the FF potential to drive the regularized wave function

$$
\psi^{(\mathrm{reg})}(\mathbf{r}, t, \lambda(t))=\phi_{n}(\mathbf{r}, \lambda(t)) e^{-i \varphi_{\mathrm{dyn}}(t)+i \varepsilon \delta \theta(\mathbf{r}, t)}
$$

finally reads (Masuda and Nakamura, 2010)

$$
\begin{aligned}
V_{F F}(\mathbf{r}, t)= & \dot{\Lambda} \varepsilon \delta V\left[\mathbf{r}, \tilde{\lambda}_{t}\right]+V_{0}\left[\mathbf{r}, \tilde{\lambda}_{t}\right] \hbar \ddot{\Lambda} \varepsilon \delta \theta\left[\mathbf{r}, \tilde{\lambda}_{t}\right] \\
& -\left.\hbar \dot{\Lambda}^{2} \varepsilon^{2} \frac{\partial \delta \theta}{\partial \lambda}\right|_{\mathbf{r}, \tilde{\lambda}_{t}}-\frac{\hbar^{2}}{2 m_{0}} \dot{\Lambda}^{2} \varepsilon^{2}(\nabla \delta \theta)^{2},
\end{aligned}
$$


D. Guéry-Odelin et al:: Shortcuts to adiabaticity: Concepts, methods, ...

$$
\begin{aligned}
0 & =\left|\phi_{n}\right|^{2} \Delta \delta \theta+2 \operatorname{Re}\left[\phi_{n} \nabla \phi_{n}^{*}\right] \cdot \nabla \delta \theta+\frac{2 m}{\hbar} \operatorname{Re}\left[\phi_{n} \partial_{\lambda} \phi_{n}^{*}\right], \\
\delta V & =-\hbar \operatorname{Im}\left[\frac{\partial_{\lambda} \phi_{n}}{\phi_{n}}\right]-\frac{\hbar^{2}}{m} \operatorname{Im}\left[\frac{\nabla \phi_{n}}{\phi_{n}}\right] \cdot \nabla \delta \theta .
\end{aligned}
$$

As an application, consider the 1D transport of a wave function with a moving confining potential $V_{0}(x, t)=$ $U(x-\varepsilon t)$. We readily find $\delta V=0$ and $\delta \theta=m x / \hbar$ for all $n$. The fast-forward potential is now $n$ independent and reads

$$
V_{F F}(x, t)=U\left(x-x_{0}(t)\right)-m x \ddot{x}_{0},
$$

with $x_{0}(t)=\varepsilon \Lambda(t)$ and terms that depend only on time have been dropped. This result is exactly the one found by the compensating force approach within invariant-based inverse engineering (Torrontegui et al., 2011), see Sec. II.C.2.b: the inertial forces in the frame attached to the moving potential are compensated for by an appropriate uniform time-dependent force.

Let us underline some conceptual and methodological similarities and differences with the CD approach: even if the adiabatic states $\phi_{n}(\mathbf{r}, \lambda(\mathbf{t}))$ are used as a reference in the construction, now the auxiliary potential in general depends on the specific $n$th wave function used (except in the previous example and for the Lewis-Leach family as discussed later), and the dynamical wave function differs along the dynamics with the adiabatic function more strongly than just by a (constant in $\mathbf{r}$ ) phase factor, because of the position-dependent phases in Eq. (81). Moreover, the FF method leads by construction to local and real potentials. By contrast, the $\mathrm{CD}$ recipe may lead to auxiliary nonlocal terms that depend on the momentum.

\section{Streamlined fast-forward approach}

The original fast-forward approach is somewhat involved. A simpler, more direct "streamlined version" of fast forward was proposed by Torrontegui, Martínez-Garaot et al. (2012) and further developed further by Torrontegui, MartínezGaraot et al. (2013) and Martínez-Garaot et al. (2016) without making explicit use of a slow reference adiabatic process. Kiely et al. (2015) extended the streamlined version for a charged particle in an electromagnetic field.

This streamlined version is essentially inverse engineering. For this discussion we use a 1D setting. The key simplification is to directly inverse engineer the potential $V(x, t)$ from the given (desired) $\psi(x, t)=\rho(x, t) e^{i \phi(x, t)}$ wave function in the Schrödinger equation, imposing $V(x, t)$ to be local,

$$
V(x, t)=\frac{1}{\psi(x, t)}\left(i \hbar \frac{\partial \psi(x, t)}{\partial t}+\frac{\hbar^{2}}{2 m} \frac{\partial^{2} \psi(x, t)}{\partial x^{2}}\right)
$$

Using the representation ( $\rho$ and $\phi$ real)

$$
\psi(x, t)=\rho(x, t) e^{i \phi(x, t)},
$$

and imposing $\operatorname{Im}[V(x, t)]=0$, we get a continuity equation

$$
\frac{1}{\rho} \frac{\partial \rho}{\partial t}+\frac{\hbar}{2 m}\left(\frac{2}{\rho} \frac{\partial \phi}{\partial x} \frac{\partial \rho}{\partial x}+\frac{\partial^{2} \phi}{\partial x^{2}}\right)=0,
$$

that links $\rho$ and $\phi$. In particular, if $\rho(x, t)$ is given, $\phi(x, t)$ cannot be arbitrary. The expression for the potential then reads

$$
V(x, t)=-\hbar \frac{\partial \phi}{\partial t}+\frac{\hbar^{2}}{2 m}\left[\frac{1}{\rho} \frac{\partial^{2} \rho}{\partial x^{2}}-\left(\frac{\partial \phi}{\partial x}\right)^{2}\right] .
$$

Equation (87) can be integrated formally,

$$
\frac{\partial \phi}{\partial x}=-\frac{m u(x, t)}{\hbar}
$$

where $u$ plays the role of a "hydrodynamic velocity,"

$$
u(x, t)=\frac{1}{\rho^{2}(x, t)} \frac{\partial}{\partial t}\left(\int_{0}^{x} \rho^{2}\left(x^{\prime}, t\right) d x^{\prime}\right) .
$$

The potential $V(x, t)$ can therefore be inferred from $\rho(x, t)$ as

$$
\begin{aligned}
V(x, t)= & m \frac{\partial}{\partial t} \int_{0}^{x} u\left(x^{\prime}, t\right) d x^{\prime}+\frac{\hbar^{2}}{2 m} \frac{1}{\rho(x, t)} \frac{\partial^{2} \rho(x, t)}{\partial x^{2}} \\
& -\frac{1}{2} m u^{2}(x, t)-\hbar \dot{\phi}_{0}(t)
\end{aligned}
$$

where $\phi_{0} \equiv \phi(x=0, t)$. This is the central result by MartínezGaraot et al. (2016).

While the freedom to choose $\rho(x, t)$ is very welcome for applications that go beyond the speedup of adiabatic processes, for example, a transfer between the ground and the first excited state of a harmonic oscillator, $\rho$ may of course also be chosen as an adiabatic function. Then connections to the other STA approaches can be made. An important fact is that for the Lewis-Leach family of Hamiltonians, the fast-forward potential becomes $n$ independent (Torrontegui, MartínezGaraot et al., 2012; Patra and Jarzynski, 2017b) and the method provides the terms found in invariant-based inverse engineering. As well, the local FF potentials may be unitarily related to the nonlocal CD Hamiltonians. Moreover, Patra and Jarzynski (2017b) noticed the following connection with CD driving: The generally state-dependent Hamiltonian

$$
H_{C D}(n)=-\frac{p u_{n}+u_{n} p}{2}
$$

where $u_{n}$ is the hydrodynamic velocity for the $n$th adiabatic state, acts on the (parallel transported, real) $\langle x \mid n(t)\rangle$ exactly as $H_{C D}$ does. They also introduced an acceleration flow field $a(x, t)=-\partial u / \partial t+u \partial u / \partial x$ so that the FF potential for a real function $\rho(x, t)$ corresponding to some eigenstate of $H_{0}$, which includes kinetic energy and a reference potential $V_{0}(x, t)$, may be written compactly as

$$
V(x, t)=V_{0}(x, t)-m \int_{0}^{x} a\left(x^{\prime}, t\right) d x^{\prime} .
$$

FF potentials may have divergences due to wave function nodes. Nevertheless, Martínez-Garaot et al. (2016) 
demonstrated that in a transition from ground to excited states, truncating the potential is a viable approximation.

\section{Generalizations and terminology}

Masuda and Rice (2016) reviewed FF and CD approaches focusing on applications in molecular systems. The FF method was extended to finite-dimensional Hilbert space with applications to drive two-level (Takahashi, 2014) and three-level systems (Masuda and Rice, 2016), or two-spin systems (Setiawan et al., 2017) and spin clusters with different geometries (Setiawan et al., 2019). In a finite Hilbert space, the auxiliary potential may be constrained by some criterion (e.g., to be diagonal). In particular, Setiawan et al. (2017) found different state-dependent CD terms.

FF was applied to manipulate Bose-Einstein condensates in optical lattices (Masuda, Nakamura, and del Campo, 2014; Masuda and Rice, 2014; Masuda, Nakamura, and Nakahara, 2018), to accelerate the STIRAP protocol (Masuda and Rice, 2015a, 2016), or to investigate the fast generation of entanglement in spin systems (Setiawan et al., 2017). The extension to the classical realm was discussed by Jarzynski et al. (2017); see Sec. VII.A.

The method was also used to control a charged quantum particle that interacts with electromagnetic fields (Masuda and Nakamura, 2011; Kiely et al., 2015; Masuda and Rice, 2015c). In Kiely et al. (2015) the fields found via invariant-based engineering and (streamlined) FF, to change the radial spread of the particle state in a Penning trap, are shown to be equivalent. Khujakulov and Nakamura (2016) and Nakamura et al. (2017) accelerated the tunneling of a charged particle treating the wave function phase differently than Masuda and Nakamura (2010).

We conclude the section with a comment on terminology. Some [see, e.g., Bukov, Sels, and Polkovnikov (2019) and Villazon, Polkovnikov, and Chandran (2019)] qualify as FF any Hamiltonian $H[\lambda(t)]$ for which no terms are added and only the parameter $\lambda$ (possibly multidimensional) is shaped in time to get to target states with unit fidelity. While this notion is in agreement with some of the results of the FF methodology previously described, it generally covers a different domain. For example, Masuda and Rice (2015a, 2016) set a discrete FF approach where actually an additional control parameter with respect to the CD driving term is added.

\section{F. FAQUAD and related approaches}

Fast quasiadiabatic (FAQUAD) and related approaches depart from the core of paradigms discussed so far in that they are intended from the start as approximate methods to balance two conflicting aims: shortening the process time and keeping the process as adiabatic as possible with respect to the actual Hamiltonian, not with respect to a reference Hamiltonian. For Hamiltonians that depend on one control parameter $\lambda(t)$, the strategy is to distribute its rate of change $\dot{\lambda}$ so that diabatic transitions are equally likely along the process. In this way $\dot{\lambda}$ slows down at and near avoided crossings but allows for fast changes away from them to make diabatic transitions weak along the whole process.
The specific methods differ on the following: the exact recipe used to distribute diabaticity "homogeneously" from $t=0$ to $t_{f}$, which leads to different $\lambda(t)$, and on the spectral information needed to implement them. This latter aspect sets a hierarchy of complementary approaches: the maximum information corresponds to FAQUAD, which needs the eigenfunctions and eigenvalues; the local adiabatic, the uniform adiabatic, or the parallel adiabatic transfer approaches imply an intermediate level which only requires eigenvalues; finally, phenomenological approaches may be based on knowing only the location of avoided crossings (Amin, 2008; Xing-Xin et al., 2013). Complex systems and, in particular, many-body systems to implement quantum adiabatic computing and quantum annealing constitute a natural domain and motivation to develop effective methods that need little or no spectral information (Albash and Lidar, 2018; Tian, 2018). Of course there is also a domain of simpler systems with more information-consuming approaches that are useful and applicable.

FAQUAD. - The idea of keeping the adiabaticity parameter constant along trap expansions, i.e., such that $\dot{\omega} / \omega^{2}=c \ll 1$, had been applied in a number of works (Kastberg et al., 1995; Chen, Ruschhaupt et al., 2010; Bowler et al., 2012; Torrontegui, Chen, Modugno, Ruschhaput et al., 2012; Martínez-Garaot et al., 2013) and was generalized for other systems in the FAQUAD approach developed by MartínezGaraot, Ruschhaupt et al. (2015). In the simplest two-level scenario with instantaneous eigenvalues $E_{1,2}(t)$ and eigenvectors $\phi_{1,2}(t)$,

$$
\hbar\left|\frac{\left\langle\phi_{1}(t) \mid \partial_{t} \phi_{2}(t)\right\rangle}{E_{1}(t)-E_{2}(t)}\right|=\hbar\left|\frac{\left\langle\phi_{1}(t)|\partial H / \partial t| \phi_{2}(t)\right\rangle}{\left[E_{1}(t)-E_{2}(t)\right]^{2}}\right|=c,
$$

and, as $\lambda=\lambda(t)$, the chain rule gives

$\dot{\lambda}=\mp \frac{c}{\hbar}\left|\frac{E_{1}(\lambda)-E_{2}(\lambda)}{\left\langle\phi_{1}(\lambda) \mid \partial_{\lambda} \phi_{2}(\lambda)\right\rangle}\right|=\mp \frac{c}{\hbar}\left|\frac{\left[E_{1}(\lambda)-E_{2}(\lambda)\right]^{2}}{\left\langle\phi_{1}(\lambda)|\partial H / \partial \lambda| \phi_{2}(\lambda)\right\rangle}\right|$,

where $\mp$ applies to a monotonous decrease or increase of $\lambda(t)$. Equation (95) must be solved with the boundary conditions $\lambda(0)$ and $\lambda\left(t_{f}\right)$, which fixes $c$ and the integration constant. This technique has been applied to accelerate processes described by two- and three-level systems such as cotunneling and splitting of two bosons in a double well, see Fig. 5, to generate macroscopically entangled states in a TonksGirardeau gas (Martínez-Garaot, Palmero et al., 2015), to design optical waveguide devices (Chung, Lee, and Tseng, 2017; Liu and Tseng, 2017; Martínez-Garaot, Muga, and Tseng, 2017), and quantum neural networks (Torrontegui and García-Ripoll, 2019).

Other approaches.-In the "parallel adiabatic transfer" technique (Guérin, Thomas, and Jauslin, 2002; Guérin, Hakobyan, and Jauslin, 2011) the level gap is required to be constant, which prevents it from being applicable when the initial and final gaps are different.

The "uniform adiabatic" method developed by Quan and Zurek (2010) relies on a comparison of transition and relaxation time scales and proposes, instead of Eq. (95), 


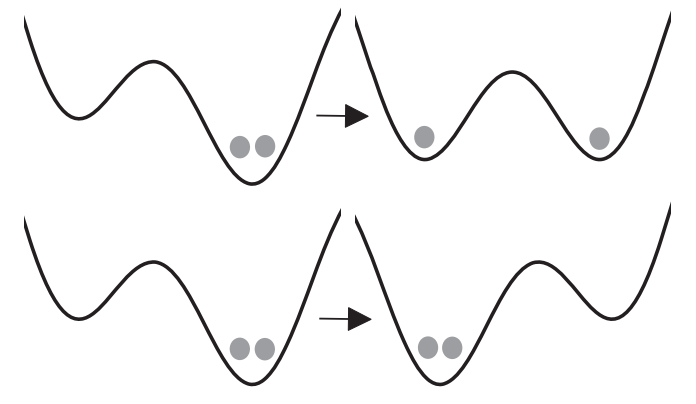

FIG. 5. Splitting and cotunneling operations on two interacting bosons performed by FAQUAD. From Martínez-Garaot, Ruschhaupt et al., 2015.

$$
\dot{\lambda}=\mp \frac{c_{U A}}{\hbar}\left|\frac{\left[E_{1}(\lambda)-E_{2}(\lambda)\right]^{2}}{\partial\left[E_{1}(\lambda)-E_{2}(\lambda)\right] / \partial \lambda}\right| .
$$

The "local adiabatic" approach (Roland and Cerf, 2002; Richerme et al., 2013) gives an equation similar to Eq. (95), without the factor $\left\langle\phi_{1}(\lambda)|\partial H / \partial \lambda| \phi_{2}(\lambda)\right\rangle$. This leads to a different constant $c$ and time dependence of the parameter $\lambda(t)$, and therefore different minimal times. There are many works applying the local adiabatic method in the context of adiabatic quantum computation (Albash and Lidar, 2018). For the Grover problem to find a marked item in an unsorted database of $N$ items, the schedule provided by the local adiabatic approach provides the best asymptotic scaling of the time needed with respect to $N$ (Albash and Lidar, 2018). Schaller, Mostame, and Schützhold (2006) generalized the local adiabaticity condition taking into account high-order powers of $E_{1}(\lambda)-E_{2}(\lambda)$. Moreover, Wiebe and Babcock (2012) and Kieferová and Wiebe (2014) proposed a method combining linear local adiabaticity and the boundary cancellation methods. In boundary cancellation methods (Morita, 2007; Rezakhani, Pimachev, and Lidar, 2010) the diabatic transitions at the time boundaries are suppressed by imposing vanishing derivatives at the boundaries $\dot{H}[\lambda(t)]=0$ at $t=0$ and $t=t_{f}$. Recently, Stefanatos and Paspalakis (2019a, 2019 b) proposed a modified FAQUAD protocol in which the adiabaticity parameter $c$ is not held constant but follows a simple "on-off" modulation found by optimal control theory.

Martínez-Garaot, Ruschhaupt et al. (2015) compared local adiabatic, uniform adiabatic, and FAQUAD approaches, also with a $\pi$ pulse and a linear ramp, for a two-level population inversion. The model uses a bare basis $|1\rangle=\left(\begin{array}{l}1 \\ 0\end{array}\right)$ and $|2\rangle=\left(\begin{array}{l}0 \\ 1\end{array}\right)$, so that a time-dependent state is $|\Psi(t)\rangle=b_{1}(t)|1\rangle+b_{2}(t)|2\rangle$ and

$$
H_{0}=\left(\begin{array}{cc}
0 & -\sqrt{2} J \\
-\sqrt{2} J & U-\Delta
\end{array}\right)
$$

where the bias $\Delta=\Delta(t)$ is the control parameter, and $U>0$, $J>0$ are constant. The goal is to drive the eigenstate from $\left|\phi_{1}(0)\right\rangle=|2\rangle$ to $\left|\phi_{1}\left(t_{f}\right)\right\rangle=|1\rangle$. To design the reference adiabatic protocol Martínez-Garaot, Ruschhaupt et al. (2015) imposed on $\Delta(t)$ the boundary conditions $\Delta(0) \gg U, J$, and $\Delta\left(t_{f}\right)=0$. The FAQUAD protocol is shown in Fig. 6(a) compared to a linear-in-time $\Delta(t)$ and a
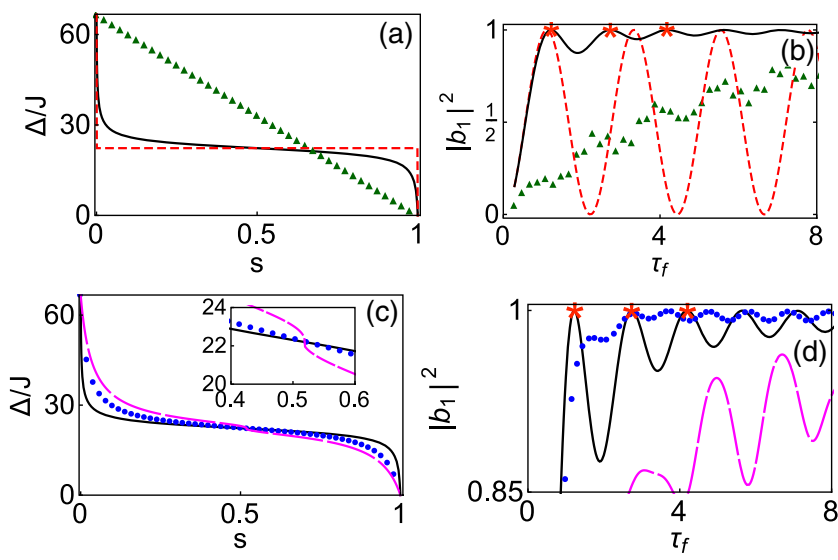

FIG. 6. (a) Bias vs $s=t / t_{f}$ for linear-in-time bias (green triangles), $\pi$ pulse (short-dashed red line), and FAQUAD (solid black line). (b) Final ground state population $\left|b_{1}\left(t_{f}\right)\right|^{2}$ vs $\tau_{f}=$ $J t_{f} / \hbar$ for linear-in-time bias (green triangles), $\pi$ pulse (shortdashed red line), and FAQUAD (solid black line). (c) Bias vs $s$ for FAQUAD (solid black line), local adiabatic approach (blue dots), and uniform adiabatic approach (long-dashed magenta line). The inset amplifies a kink of the uniform adiabatic approach. (d) $\left|b_{1}\left(t_{f}\right)\right|^{2}$ vs $\tau_{f}=J t_{f} / \hbar$ for FAQUAD (solid black line), local adiabatic approach (blue dots), and uniform adiabatic approach (long-dashed magenta line). The stars in (b) and (d) correspond to integer multiples of the characteristic FAQUAD timescale $2 \pi / \Phi . \Delta(0) / J=66.7, U / J=22.3$. From Martínez-Garaot, Ruschhaupt et al., 2015.

constant $\Delta=U$. The final ground state populations $\left|b_{1}\left(t_{f}\right)\right|^{2}$ versus the dimensionless final time $\tau_{f}=J t_{f} / \hbar$ are shown in Fig. 6(b). For $\Delta=U$ between 0 and $t_{f}$, Rabi oscillations occur [short-dashed red line in Fig. 6(b)] due to interference between two dressed states. By contrast the FAQUAD process is quasiadiabatic and is dominated by one dressed state. The existence of special process times with perfect fidelity can be also understood as an interference phenomenon with characteristic period $T=2 \pi / \Phi$, the minimal time for fidelity one, where $\Phi=\left(\hbar / t_{f}\right) \int_{0}^{t_{f}} d t E_{\text {gap }}(t)$ and $E_{\text {gap }}$ is the gap between instantaneous levels. Figure 6(b) also shows the poorer results of the linear ramp for $\Delta(t)$. Figures $6(\mathrm{c})$ and $6(\mathrm{~d})$ compare FAQUAD, local adiabatic, and uniform adiabatic approaches. FAQUAD gives the best behavior at short times, and the local adiabatic method achieves better population stability after a few oscillations.

\section{G. Optimal control and shortcuts to adiabaticity}

Optimal control theory is a widely used method (Pinch, 1993; Kirk, 2004) to find control parameter trajectories that minimize a given cost function (global constraint) and obey some specific boundary conditions. According to the Pontryagin maximum principle, such extremal solutions satisfy the equations of a generalized Hamiltonian system. When more constraints are imposed, the quantity to be minimized should be adapted with some weights for the different constraints. Optimal control theory analytically yields solutions only for low-dimensional systems. Very often the OCT solutions are found by discretizing the problem and 
implementing numerical approaches such as dynamic programming, the gradient ascent, or Krotov algorithms.

In contrast, STA techniques are not built, in general, upon a minimization principle. However, they serve a similar objective, to drive the system toward the desired states in a short amount of time. The solutions found for the parameter trajectories are by construction typically analytical, continuous, and well adapted to introduce many local constraints (for instance the successive derivative at the initial and final time of the dynamical quantity of interest). Directly comparing both approaches is therefore somewhat misleading. Actually, a class of STA solutions depending on a free parameter can be used to minimize a given cost function yielding results close to those of optimal control theory.

In fact there are many examples in which STA and OCT methods are usefully combined to get nearly optimal protocols by minimizing a cost function of interest or to accommodate for extra constraints in a reduced space made of analytical solutions originating from an STA approach. Such a hybrid strategy has been explicitly worked out to engineer spinlike systems (Hegerfeldt, 2013; Sun, Saxena, and Sinitsyn, 2017; B. B. Zhou et al., 2017), to minimize final excitation after a fast transport in the presence of anharmonicities (Torrontegui, Chen, Modugno, Schmidt et al., 2012; Zhang et al., 2016), to ensure fast transport with extra relevant constraints (e.g., minimum transient energy, bounded trap velocity, or bounded distance from the trap center) (Chen et al., 2011; Torrontegui et al., 2011; Stefanatos and Li, 2014; Alonso et al., 2016; Amri et al., 2018), to ensure a fast and robust shuttling of an ion with noise (Lu, Muga et al., 2014), to perform fast expansions (Salamon et al., 2009; Stefanatos, Ruths, and Li, 2010; Stefanatos, 2013, 2017b; Lu, Chen et al., 2014; Boldt, Salamon, and Hoffmann, 2016; Plata et al., 2019), or to drive a many-body Lipkin-Meshkov-Glick system (Campbell et al., 2015).

Mortensen et al. (2018) combined the perturbative approach to enhance robustness described in Sec. II.H with optimal control. A cost function is defined to achieve low peak laser power and stability against systematic error (or scaling) of the control functions. Shortcut schemes for the $\Lambda$ system were found which minimize this function. This strategy is less computationally expensive than other optimal control methods, since the Schrödinger equation does not need to be solved for every run. Normally the fidelity is part of the cost function, but this is unnecessary for STA methods.

To transport a particle in a harmonic trap of angular frequency $\omega_{0}$, OCT provides a minimum transport time $t_{f}$ for a fixed transport distance $d$ using a "bang-banglike" solution for which the acceleration is changed abruptly from a constant value $\omega_{0}^{2} \delta$ to the opposite value $-\omega_{0}^{2} \delta$ at $t_{f} / 2$ and where the relation between the final time and the parameters is $t_{f}=\left(2 / \omega_{0}\right)(d / \delta)^{1 / 2}$ (Chen et al., 2011). This kind of solution was implemented experimentally to transport a cold cloud of atoms trapped in a moving optical tweezer in twice the oscillation period (Couvert et al., 2008).

The challenges and prospects of optimal control theory for quantum systems which overlap with those of shortcuts to adiabaticity are discussed in the review articles by Glaser et al. (2015) and Koch (2016).

\section{H. Robustness}

Adiabatic processes possess a natural robustness to parameter variations. As long as the parameters vary slowly enough, there are many smooth adiabatic paths to the same final result, ignoring phases. However, this robustness does not apply to all imperfections, for example, adiabatic wave packet splitting is very sensitive to asymmetries in the potential (Torrontegui, Martínez-Garaot et al., 2013). Indeed adiabatic drivings are prone to decoherence, excitations, and particle loss, due to the accumulation of noisy perturbations during long process times. STA methods prove useful as they reduce the detrimental cumulative effect of noise, but they require specific control of the parameters for intermediate times, so in general will not have such a natural stability against smooth parameter variations.

Nevertheless, the flexibility of STA methods can be exploited to improve robustness against external influences and imperfections. Within the class of control schemes that work perfectly in the ideal, noiseless setting, the objective is to find the most robust one versus the relevant imperfections or noises, singled out or combined, of a given experiment.

\section{Error sensitivity and its optimization using perturbation theory}

In several works [see, e.g., Choi, Onofrio, and Sundaram (2012) and Torrontegui, Chen, Modugno, Schmidt et al. (2012)], the effect of perturbations and imperfections on STA protocols is analyzed. We can go further and actively improve or even maximize the robustness of the control schemes.

The starting point to do so (Ruschhaupt et al., 2012) is to first design a class of shortcut schemes which fulfill the wanted control task with fidelity one without perturbations. The next step is to define an error sensitivity $q \geq 0$ with respect to the relevant error source; this is done by using perturbation theory to define a series expansion of the fidelity $F(\lambda)$ in terms of the error parameter, $q$ being (minus) the coefficient of the quadratic term. Depending on the nature of the error, systematic or stochastic, $q$ may be found using a Schrödinger equation or a master equation.

This principle was applied to many physical systems and different sources of errors and imperfections. In the following, we review some of these works beginning with the simple example of a two-level system.

\section{a. Illustrative example: Control of a two-level system}

Ruschhaupt et al. (2012) examined population inversion in a two-level quantum system subjected to different systematic and noisy errors. The starting point is a two-level Hamiltonian

$$
H_{0}(t)=\frac{\hbar}{2}\left(\begin{array}{cc}
-\Delta(t) & \Omega_{R}(t)-i \Omega_{I}(t) \\
\Omega_{R}(t)+i \Omega_{I}(t) & \Delta(t)
\end{array}\right) .
$$

Following the invariant-based inverse engineering, see Sec. II.C.2, the control parameters $\Omega_{R}(t), \Omega_{I}(t)$, and $\Delta(t)$ can be calculated from the auxiliary functions $\theta, \alpha$, and $\gamma$ via Eqs. (52), (53), and (54), with the boundary conditions $\theta(0)=0$ and $\theta(T)=\pi$. By realizing these control functions exactly the population would be inverted in the unperturbed, 
error-free case along a family of solutions for the parameter paths.

For systematic errors, for example, if atoms at different positions are subjected to slightly different fields due to the Gaussian profile of the laser, the actual, experimentally implemented Hamiltonian is $H_{0}+\beta H_{1}$, where $H_{1}(t)=$ $\left.H_{0}(t)\right|_{\Delta \equiv 0}$ and $\beta$ is the dimensionless amplitude of the relative systematic error in $\Omega_{R}$ and $\Omega_{I}$. To give a specific example, consider now only systematic errors in the Rabi frequency. Using time-dependent perturbation theory, the population to be in the excited state $P_{2}(\beta)$ can be expressed as $P_{2}(\beta)=P_{2}(0)-q_{S} \beta^{2}+\cdots$, where the noise sensitivity is found to be

$$
q_{S}=-\left.\frac{1}{2} \frac{\partial^{2} P_{2}}{\partial \beta^{2}}\right|_{\beta=0}=\left|\int_{0}^{t_{f}} d t e^{-i \gamma} \dot{\theta} \sin ^{2} \theta\right|
$$

One simple choice that gives $q_{S}=0$ is

$$
\begin{aligned}
\Omega_{R} & =\frac{\pi}{t_{f}} \sqrt{1+16 \sin ^{6}\left(\frac{\pi t}{t_{f}}\right)}, \quad \Omega_{I}=0, \\
\Delta & =-\frac{8 \pi}{t_{f}} \sin \left(\frac{\pi t}{t_{f}}\right) \sin \left(\frac{2 \pi t}{t_{f}}\right) \frac{1+4 \sin ^{6}\left(\pi t / t_{f}\right)}{1+16 \sin ^{6}\left(\pi t / t_{f}\right)} .
\end{aligned}
$$

We can now consider amplitude noise. We assume that $\Omega_{R}$ and $\Omega_{I}$ are affected independently with the same strength parameter $\lambda^{2}$. This is motivated by the assumption that in principle two lasers may be used to implement the real and imaginary parts of the Rabi frequency with the same intensity. The corresponding master equation with only noise error (no systematic error) is

$$
\begin{aligned}
\frac{d}{d t} \rho= & -\frac{i}{\hbar}\left[H_{0}, \rho\right] \\
& -\frac{\lambda^{2}}{2 \hbar^{2}}\left(\left[H_{2 R},\left[H_{2 R}, \rho\right]\right]+\left[H_{2 I},\left[H_{2 I}, \rho\right]\right]\right),
\end{aligned}
$$

where $H_{2 R}(t)=\left.H_{0}(t)\right|_{\Delta \equiv \Omega_{I} \equiv 0}$, and $H_{2 I}(t)=\left.H_{0}(t)\right|_{\Delta \equiv \Omega_{R} \equiv 0}$. Note that STA methods for systems explicitly coupled to an external bath are discussed in further detail in Sec. VII.D. The noise sensitivity $q_{N}$ is defined as

$$
q_{N}:=-\left.\frac{\partial P_{2}}{\partial\left(\lambda^{2}\right)}\right|_{\lambda=0},
$$

where $P_{2}=\left\langle 2\left|\rho\left(t_{f}\right)\right| 2\right\rangle$ is the probability of the excited state at final time $t_{f}$, i.e., $P_{2} \approx 1-q_{N} \lambda^{2}$. Using time-dependent perturbation theory for the master equation, this sensitivity can again be calculated in terms of the auxiliary functions which define the invariant and the state evolution. The transient values of these functions can then be optimally chosen to minimize $q_{N}$, while keeping the boundary conditions fixed to ensure perfect state transfer without noise.

In Fig. 7, two STA schemes optimized for noise-error, respectively, systematic error are shown; see Ruschhaupt et al. (2012) for details. Clearly, different sources of imperfection need different optimized STA schemes. For a recent combination of this perturbative approach with optimal control

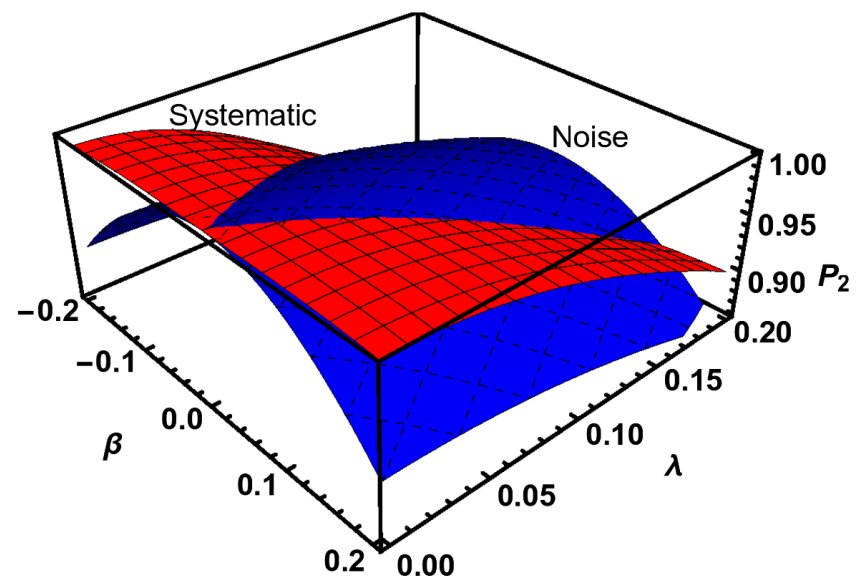

FIG. 7. Excitation probability $P_{2}$ vs noise error (strength $\lambda$ ) and systematic error parameter (strength $\beta$ ). Noise error optimized STA (blue) and systematic error optimized STA (red). Adapted from Ruschhaupt et al., 2012.

theory in the context of topologically protected gates see Ritland and Rahmani (2018).

b. Optimization using perturbation theory in other settings

The optimization of robustness of shortcut schemes using the perturbative approach has been applied to many different systems and systematic error and noise types. Dephasing noise and systematic frequency shift for the two-level system have been examined by Lu et al. (2013), where $\Gamma_{d}:=\gamma_{d} \sigma_{z}$ is the noise operator (Sarandy, Duzzioni, and Moussa, 2007), whereas Ruschhaupt and Muga (2014) addressed bit-flip noise, with $\Gamma_{b}:=\gamma_{b} \sigma_{x}$ being the noise operator. The perturbative approach has also been used to work out stable singleand two-qubit gates (Santos, 2018), and to designed schemes to suppress unwanted transitions (Kiely and Ruschhaupt, 2014; Yu et al., 2018; Y. Yan et al., 2019). It may also be combined with optimal control theory (Mortensen et al., 2018); see Sec. II.G. There are as well many works studying and improving robustness in transport problems with respect to anharmonicities (Chen et al., 2011; Torrontegui et al., 2011; Zhang, Chen, and Guéry-Odelin, 2015; Zhang et al., 2016), and noise (Lu, Muga et al., 2014; Lu, Ruschhaupt, and Muga, 2018); see Sec. III.A.

The results of Ruschhaupt et al. (2012) were extended by Daems et al. (2013) in two ways. First, higher derivatives in the error were also considered, and second, the optimization of the auxiliary function was done by starting with an Ansatz with free parameters which were optimized numerically [while it was still possible to derive the optimal scheme by Ruschhaupt et al. (2012) analytically]. In addition, the absolute systematic error in the detuning was examined. The results of Daems et al. (2013) for population inversion have been used experimentally to rephase atomic coherences in a $\mathrm{Pr}^{3+}: \mathrm{Y}_{2} \mathrm{SiO}_{5}$ crystal (Van-Damme et al., 2017) and also applied to create a superposition state with a controlled relative phase in a two-level system (Ndong et al., 2015).

Dirac systems.-An application of the perturbative technique was demonstrated for Dirac systems (Song et al., 2017). 
D. Guéry-Odelin et al.: Shortcuts to adiabaticity: Concepts, methods, ...

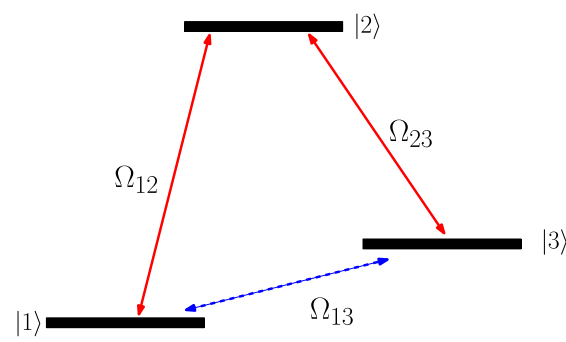

(a)

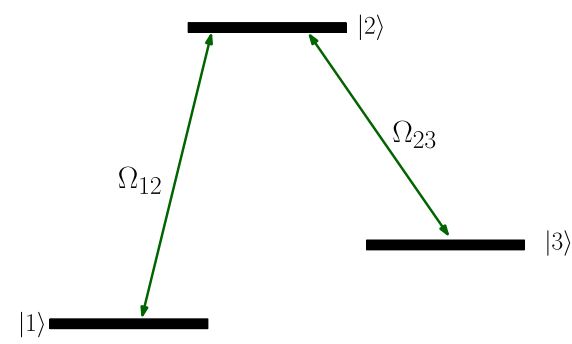

(b)

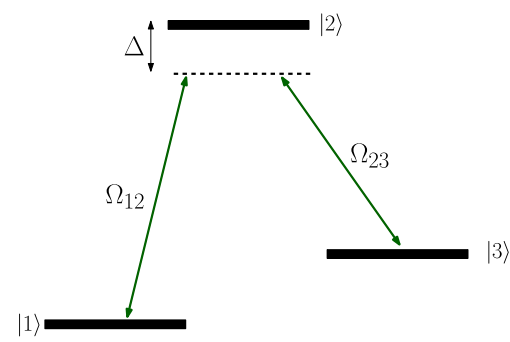

(c)

FIG. 8. Different strategies for STA in three-level systems. (a) Apply counterdiabatic STA: initial couplings (red, solid lines), additional required STA coupling (blue, dotted line). (b) Apply invariant-based inverse engineering STA: modified STA couplings (green, solid lines). (c) Detuned couplings: use STA techniques after mapped to a two-level system: modified STA couplings (green, solid lines).

A plethora of natural or artificial systems obey the Dirac equation in certain conditions, with a proper reinterpretation of symbols. The new physical platforms for Dirac dynamics (trapped ions, optics, superconducting circuits) are easier to manipulate than relativistic particles. In trapped ions, for example, the effective (simulated) mass, speed of light, or electric field may be changed in time. This rich simulation scenario opens prospects for finding and implementing new or exotic effects and carrying out fundamental studies. Shortcuts to adiabaticity offer a suitable framework for the task (Muga, Simón, and Tobalina, 2016). For example, Deffner (2016) used the fast-forward technique to suppress production of pairs (transitions among positive and negative energy solutions) in fast processes.

The goal of Song et al. (2017) was instead to induce a fast and robust population inversion among the bare levels on a $(1+1)$-dimensional Dirac equation for a charged particle simulated by ultracold trapped ions, designing a simulated electric field $\alpha_{t}$. The problem is that the coupling between momentum and internal levels in the Dirac equation changes with the momentum. For each plane wave, there is a momentum-dependent Hamiltonian,

$$
H_{p_{0}}=\left(\begin{array}{cc}
m c^{2} & c p_{0}+\alpha_{t} \\
c p_{0}+\alpha_{t} & -m c^{2}
\end{array}\right)
$$

but a robust population inversion should be independent of the momentum within the momentum spread of the wave packet. This is achieved by considering the $p_{0}$-dependent part in Eq. (102) as a perturbation and designing the time dependences of the other elements in the Hamiltonian using the approach by Ruschhaupt et al. (2012).

\section{Other approaches}

Guéry-Odelin and Muga (2014) used a Fourier method to find transport protocols (for either a single particle or BEC) which are robust with respect to spring-constant errors; see more details in Sec. VII.B.2. It exploits the fact that the final excitation energy can be expressed as the Fourier transform of the trap acceleration (Reichle et al., 2006). It avoids perturbation approximations and can also be applied to transport noninteracting particles of different species. Its connection to flatness based control in mathematics is discussed in Sec. VII.B.2.
The use of perturbation theory or iterative methods is also avoided by Zhang, Chen, and Guéry-Odelin (2017) and Levy et al. (2018). Using the fast-forward approach (see Sec. II.E) a magnetic field is determined which prescribes specific stable dynamics of a single or two interacting spins (Zhang, Chen, and Guéry-Odelin, 2017). Levy et al. (2018) designed protocols for both a two-level system and a harmonic oscillator, which are stable against Markovian noise sources. They are designed using dynamical invariants and are made robust by enforcing that the invariant approximately commutes with the noise operator during the process.

The effect of the environment can also be reduced by choosing the phases in the noise-free evolution operator $U(t)=\sum_{n} e^{i \xi_{n}(t)}|n(t)\rangle\langle n(0)|$ to design the driving Hamiltonian (Santos and Sarandy, 2018).

Boyers et al. (2018) proposed protocols based on "Floquet engineering" with periodic drivings, where the coefficients are adjusted by matching an effective Floquet Hamiltonian found by a Magnus expansion with CD-driving Hamiltonians. For a qubit inversion the method is resilient to noise because the spectral bandwidth of the protocol (centered around the Floquet frequency) is separated from the spectral bandwidth of the noise around zero as long as the noise is perturbative with respect to the driving.

\section{Three-level systems}

Few-level models are essential to understand and manipulate actual or artificial atoms. We have presented several examples of shortcuts applied to two-level models and in this section we review three-level models. STIRAP is a basic adiabatic method of reference [see Vitanov et al. (2017) for a recent review] to transfer population among the two ground states of a $\Lambda$ configuration (or the extreme states in ladder systems) without populating the excited state. Being an adiabatic process, it can be sped up with STA techniques. Here are three possible STA approaches to STIRAP speedup; see Fig. 8.

\section{Apply counterdiabatic shortcuts to the full three-level $\Lambda$ system}

We assume a three-level $\Lambda$ system consisting of two "ground levels" $|1\rangle$ and $|3\rangle$ and a central excited level $|2\rangle$ coupled with time-dependent terms $\Omega_{12}$ and $\Omega_{23}$. We refrain 
from specifying by now the exact nature of these couplings which will depend very much on the system. The first strategy is to apply the counterdiabatic STA technique directly to this $\Lambda$ system to speed up the STIRAP transfer. This strategy leads to an additional coupling between the two levels $|1\rangle$ and $|3\rangle$ (Unanyan et al., 1997; Demirplak and Rice, 2003; Chen, Lizuain et al., 2010). The improved robustness of STA schemes compared with different STIRAP protocols was shown by Giannelli and Arimondo (2014), and the effect of decay and dephasing was studied by Issoufa and Messikh (2014), who observed that the latter has more effect on the final fidelity than the former. The robustness of this scheme with respect to energies fluctuations, e.g., due to collisions of a solute with a solvent, was examined by Masuda and Rice (2015b).

In practice the additional coupling $\Omega_{13}$ can be implemented in some but not in all systems, e.g., because of selection rules due to symmetry of the states or the necessary phase of the term. In fact, Vitanov and Drewsen (2019) proposed a method for efficient optical detection and separation of chiral molecules based on the phase sensitivity of the approach.

An example of a physical system to which this first strategy was applied is "spatial adiabatic passage" (Menchon-Enrich et al., 2016) in which three wells play the role of the three internal states. The additional imaginary coupling may be implemented using a magnetic field that induces a complex tunneling term (Benseny et al., 2017). In nitrogen-vacancy electronic spins, this additional coupling was experimentally implemented mechanically via a strain field (Amezcua, Golter, and Wang, 2017; Kölbl et al., 2019). In a superconducting transmon with a three-level ladder configuration, the auxiliary field to induce a fast transition from the ground to the second excited state was achieved with a two-photon microwave pulse to circumvent the forbidden transition (Vepsäläinen, Danilin, and Paraoanu, 2018).

As a generalization, a discrete FF approach can be set to accelerate the STIRAP protocol with an additional control parameter with respect to the CD solution (Masuda and Rice, 2015a, 2016). The following text discusses alternative STA routes when the new required coupling is not easy to implement or too weak, e.g., a magnetic dipole transition.

\section{Applying invariant-based inverse engineering shortcuts to the full three-level $\Lambda$ system}

The second strategy is to apply the invariant-based inverse engineering to the $\Lambda$ system. Chen and Muga (2012) applied this strategy to a Hamiltonian with resonant couplings that imply an $S U(2)$ dynamical symmetry and built different protocols that may or may not populate level $|2\rangle$, without the need for an additional coupling between $|1\rangle$ and $|3\rangle$. Interestingly, to achieve the same fidelity, less intensity is required when the intermediate level $|2\rangle$ is populated. This means that protocols that populate level $|2\rangle$ may be considered as useful alternatives for certain systems and sufficiently short process times. Moreover, Chen and Muga (2012) put forward the concept of invariant-based "multimode driving," where the dynamical state is a combination of invariant eigenstates rather than just one of them as had been customary in previous works.
Related to this approach are also the speeded-up STIRAP protocols based on the dressed-state approach by Baksic, Ribeiro, and Clerk (2016), which were used in experiments with nuclear spins (Coto et al., 2017; B. B. Zhou et al., 2017).

\section{Using STA techniques after mapping to a two-level system}

The third strategy is based on mapping or approximate the three-level system to a two-level system and then applying two-level STA techniques. In particular, when the middle level $|2\rangle$ is detuned it can be adiabatically eliminated. The counterdiabatic techniques of STA can then be applied to this effective two-level system and the resulting pulses can be mapped back to the three-level system. No additional coupling is required between the metastable states $|1\rangle$ and $|3\rangle$, and the existing couplings are only modified. This approach was exploited theoretically by Li and Chen (2016) and, using cold

${ }^{87} \mathrm{Rb}$ atoms, experimentally implemented by Du et al. (2016).

\section{J. Motional states mapped into a discrete system}

In trapped systems a simplifying route to apply shortcuts to control motional degrees of freedom is to first discretize the quantum system into a finite number of localized states that could be time dependent. Then the previous methods can be applied. Ideally the resulting STA protocol should be translated to the original setting to check its performance, or resistance to noise and perturbations, although this step is not always realized.

We provide here some examples of approximations in terms of two, three, and four states.

- Two states: Wave packet splitting operations were modeled by systems of two time-dependent states by Torrontegui, Martínez-Garaot et al. (2013), and multiplexing or demultiplexing of harmonic oscillator vibrational states by Martínez-Garaot et al. (2013). Two-level models are also used to study spin dynamics in a quantum dot with spin-orbit coupling (SOC) (Ban et al., 2012b).

- Three states: In spatial adiabatic passage, analogous to STIRAP (Vitanov et al., 2017), a particle may tunnel between three wells. The system is approximated by a three-state system and shortcuts may be applied (Benseny et al., 2017). Martínez-Garaot et al. (2014) provided other examples, such as wave packet splitting in three wells or operations on twointeracting bosons in two wells.

- Four states: Kiely et al. (2016) and Kiely, Muga, and Ruschhaupt (2018) modeled the motion of an ultracold atom in a lattice by a four-state system and applied invariant-based STA techniques to create exotic angular momentum states of ultracold atoms in an optical lattice. In Li, Chen et al. (2018) the four-level model takes into account both motional and internal aspects, representing up and down spin states in two different wells.

\section{APPLICATIONS IN QUANTUM SCIENCE AND TECHNOLOGY}

This section is organized by system type. A number of tables group together articles, otherwise dispersed in different sections, according to transversal criteria: "gates" in Table II, 
D. Guéry-Odelin et al.: Shortcuts to adiabaticity: Concepts, methods, ...

TABLE II. Quantum logic gates designed using STA methods. CD: counterdiabatic driving; OCT: optimal control theory; QED: quantum electrodynamics; QZD: quantum Zeno dynamics; NV: nitrogen vacancy.

\begin{tabular}{|c|c|c|c|}
\hline Reference & Gate type & System & Method \\
\hline Martinis and Geller (2014) & Phase & Superconducting Xmon transmon & Optimization \\
\hline Song, Xia, and Song (2014) & Toffoli & Cavity QED & QZD + invariants \\
\hline Santos and Sarandy (2015) & Universal gates & $N$ qubits & $\mathrm{CD}$ driving \\
\hline $\begin{array}{l}\text { Chen, Xia, Chen, } \\
\text { and Song (2015) }\end{array}$ & Phase & Cavity QED & QZD + invariants \\
\hline Liang, Wu et al. (2015) & Phase & Cavity QED & QZD + invariants \\
\hline Liang, Song et al. (2015) & CNOT & Cavity QED & QZD + invariants \\
\hline Liang, Ji et al. (2015) & Swap & Cavity QED & QZD + invariants \\
\hline Zhang et al. (2015) & Non-Abelian geometric & Superconducting transmon & $\mathrm{CD}$ driving \\
\hline $\begin{array}{l}\text { Santos, Silva, } \\
\text { and Sarandy (2016) }\end{array}$ & $N$ qubit & Four-level & $\mathrm{CD}$ driving \\
\hline $\begin{array}{l}\text { X.-K. Song, Zhang, } \\
\text { Ai et al. (2016) }\end{array}$ & One- and two-qubit holonomic & $\mathrm{N} V$ centers & CD driving \\
\hline Liang et al. (2016) & Non-Abelian geometric & $\mathrm{N} V$ centers & CD driving \\
\hline Palmero et al. (2017) & Two-qubit phase & Two trapped ions & Invariants \\
\hline Du et al. (2017) & Non-Abelian geometric & $\mathrm{N} V$ centers & $\mathrm{CD}$ driving \\
\hline Wu, Ji, and Zhang (2017a) & CNOT & Cavity QED & QZD + dressed-state scheme \\
\hline Santos (2018) & Single- and two-qubit & Two- and four-level & Inverse engineering \\
\hline Liu et al. (2018) & Non-Abelian geometric & $\mathrm{N} V$ centers & Invariants \\
\hline Wang et al. (2018) & Single-qubit & Superconducting Xmon qubit & $\mathrm{CD}$ driving \\
\hline Shen and Su (2018) & Two-qubit controlled phase & Two Rydberg atoms & Invariants \\
\hline Ritland and Rahmani (2018) & Majorana & Top transmon & OCT for noise canceling \\
\hline Li, Chen et al. (2018) & One-qubit gate and transport & Double quantum dot & Inverse engineering \\
\hline T. Yan et al. (2019) & Non-Abelian geometric & Superconducting Xmon qubit & $\mathrm{CD}$ driving \\
\hline Lv et al. (2019) & Noncyclic geometric & Two-level atom & $\mathrm{CD}$ driving \\
\hline Santos et al. (2019) & Single-qubit & Nuclear magnetic resonance & CD driving \\
\hline Qi and Jing (2019) & Single- and double-qubit holonomic & Rydberg atoms & $\mathrm{CD}$ driving \\
\hline
\end{tabular}

LMG-like models in Table III, "transport" in Table IV, and "experiments" in Table V.

\section{A. Trapped ions}

Trapped ions constitute one of the most developed physical platforms to implement fundamental quantum phenomena and quantum information processing. Since many ions in a single trap are difficult to control, a way toward large-scale computations with many qubits is a divide-and-conquer scheme (Wineland et al., 1998; Kielpinski, Monroe, and Wineland, 2002), where ions are shuttled around in multisegmented Paul traps, while keeping just a few ions in each processing site. Apart from shuttling, complementary operations such as separating and merging ion chains, trap rotations, and expansions or compressions of ion chains are needed. Coulomb interactions and controllable external effective potentials created by radio frequency or dc electrodes determine the motion of the ions and the corresponding Hamiltonians, which can be approximated by quadratic forms near equilibrium.

\section{Dynamical normal modes}

Dynamical normal modes are a useful generalization of ordinary normal modes for time-dependent, quadratic Hamiltonians (Palmero et al., 2014) or in the small-oscillations regime for nonharmonic ones. They are independent harmonic motions which describe the dynamics of an effective timedependent harmonic oscillator. Dynamical normal modes help to describe the motion in a simple way but also to inverse engineer the potentials to achieve fast motions without final excitation. The invariant-based engineering of the time

TABLE III. Works where STA methods are applied to LMG-like models.

\begin{tabular}{|c|c|c|}
\hline Reference & Reference Hamiltonian & Comment \\
\hline Juliá-Díaz et al. (2012) & $U(t) J_{z}^{2}-2 J J_{x}$ & Invariants, large $N$ \\
\hline Yuste et al. (2013) & $U J_{z}^{2}-2 J(t) J_{x}$ & Invariants, time-dependent mass \\
\hline Takahashi (2013b) & $-(2 / N)\left[J_{x}(t) S_{x}^{2}-J_{y}(t) S_{y}^{2}+\gamma S_{y}^{2}\right]-2 h(t) S_{z}$ & Large $N, \mathrm{CD}$ \\
\hline Campbell et al. (2015) & $-(2 / N)\left(S_{x}^{2}+\gamma S_{y}^{2}\right)-2 h(t) S_{z}$ & Approximate CD \\
\hline Opatrný et al. (2016) & $A_{c}(t) H_{c}+A_{n}(t) H_{n} ; H_{c}=\vec{c} \cdot \vec{J}, H_{n}=\left(J_{z}-n I\right)^{2}$ & Approximate $\mathrm{CD}$ \\
\hline Hatomura (2017) & $-[(2 J) / N] S_{z}^{2}-2 \Gamma(t) S_{x}-2 h S_{z}$ & Mean field, CD \\
\hline Takahashi (2017b) & $\begin{array}{l}f(t)\left\{-[(2 J) / N] S_{z}^{2}-2 h S_{z}\right\}-2 \Gamma(t) S_{x} \\
-f(t)[(2 J) / N] S_{z}^{2}-2 \Gamma_{x}(t) S_{x}-2 \Gamma_{y}(t) S_{y} \\
f(t)\left(-\sum J_{i j} \sigma_{i} \sigma_{i}^{2}-2 h S_{z}\right)-2 \Gamma(t) S_{x}\end{array}$ & Mean field, invariants \\
\hline Hatomura (2018) & $-[(2 J) / N] S_{z}^{2}-2 \Gamma_{x}(t) S_{x}$ & Mean field, CD \\
\hline
\end{tabular}


D. Guéry-Odelin et al:: Shortcuts to adiabaticity: Concepts, methods, ...

TABLE IV. List of papers on STA-mediated transport. CD: counterdiabatic driving; IE: inverse engineering; OCT: optimal control theory; FF: fast forward; Exp.: experiment.

\begin{tabular}{|c|c|c|}
\hline Reference & Method & System \\
\hline Couvert et al. (2008) & Fourier-based IE (Exp.) & Cold atoms in moving optical tweezers \\
\hline Masuda and Nakamura (2010) & FF & 1 particle in arbitrary trap \\
\hline Torrontegui et al. (2011) & $\begin{array}{l}\text { Invariants, compensating-force, } \\
\text { bang-bang }\end{array}$ & 1 particle \\
\hline Chen et al. (2011) & Invariants + OCT & 1 particle harmonic transport \\
\hline $\begin{array}{l}\text { Torrontegui, Chen, Modugno, } \\
\text { Schmidt et al. (2012) }\end{array}$ & Invariants $+\mathrm{OCT}$ & BEC \\
\hline Sun et al. (2012) & Bang-bang (Exp.) & $\begin{array}{l}\text { Load in a } 2 \mathrm{D} \text { planar overhead } \\
\text { mechanical crane }\end{array}$ \\
\hline Bowler et al. (2012) & Fourier transform (Exp.) & 1,2, or 9 ions in Paul trap \\
\hline Walther et al. (2012) & Optimized drivings (Exp.) & 1 or 2 ions in Paul trap \\
\hline S. Ibáñez et al. (2012) & Unitary transformations & 1 particle in harmonic trap \\
\hline Palmero et al. (2013) & Invariants & 2 ions in anharmonic traps \\
\hline Stefanatos and $\mathrm{Li}$ (2014) & OCT & 1 particle \\
\hline Lu, Muga et al. (2014) & Invariants + perturbation theory + OCT & 1 ion \\
\hline Fürst et al. (2014) & OCT + compensating force & 1 ion in Paul trap \\
\hline Palmero et al. (2014) & Invariants & Mixed-species ion chains in Paul trap \\
\hline Guéry-Odelin and Muga (2014) & Fourier method & 1 particle or BEC \\
\hline Pedregosa-Gutierrez et al. (2015) & Numerical simulations & Large ion clouds \\
\hline Lu et al. (2015) & Invariants & 2 ions of different mass \\
\hline Zhang, Chen, and Guéry-Odelin (2015) & Inverse engineering & 1 particle in anharmonic trap \\
\hline Kamsap et al. (2015) & Numerical simulations & Large ions clouds \\
\hline Martínez-Garaot, Palmero et al. (2015) & FAQUAD, compensating-force & 1 particle \\
\hline Alonso et al. (2016) & Bang-bang (Exp.) & 1 ion \\
\hline Zhang et al. (2016) & Invariants + OCT & Cold atoms \\
\hline Okuyama and Takahashi (2016) & Lax pairs + local CD & Solitonlike potentials \\
\hline An et al. (2016) & $\mathrm{CD}$ and $\mathrm{CD}+$ unitary transformation (Exp.) & Simulated transport of 1 ion \\
\hline Funo et al. (2017) & $\mathrm{CD}$ & 1 ion in Paul trap \\
\hline Tobalina et al. (2017) & Invariants & 1 ion in a nonrigid trap \\
\hline Li, Zhang, and Chen (2017) & Trigonometric protocols & Cold atoms in anharmonic traps \\
\hline Torrontegui et al. (2017) & Invariants & Load in mechanical crane \\
\hline Dowdall et al. (2017) & Pauli blocking & Ultracold Fermi gases \\
\hline González-Resines et al. (2017) & Invariants & Load in mechanical crane \\
\hline Corgier et al. (2018) & Inverse engineering & BECs in atom chips \\
\hline Kaufmann et al. (2018) & Invariants (Exp.) & 1 ion in Paul trap \\
\hline Lu, Ruschhaupt, and Muga (2018) & Invariants & 1 ion \\
\hline Tobalina, Alonso, and Muga (2018) & OCT + compensating-force & 1 ion in Paul trap \\
\hline Ness et al. (2018) & Invariants (Exp.) & Cold atoms in optical dipole trap \\
\hline Chen, Jiang et al. (2018) & Inverse engineering & Spin-orbit-coupled BECs \\
\hline Li, Chen et al. (2018) & Inverse engineering & Qubit in double quantum dots \\
\hline Amri et al. (2018) & OCT \& STA & BECs in atom chips \\
\hline
\end{tabular}

dependence of the potentials is a natural route to that end. An important difference with respect to inverse engineering a single harmonic oscillator is that several time-dependent harmonic oscillators for the different dynamical modes have to be engineered simultaneously with common control functions.

For example, to transport a chain of two ions, the position of the external harmonic trap is a common control function, and we cannot engineer a different trap position for each mode. The way to solve this type of inversion problem is to increase the number of adjustable parameters in the Ansätze for the auxiliary functions, see Sec. II.C.2.b, so that all the boundary conditions of the auxiliary functions of all modes are satisfied simultaneously, either exactly or via minimization subroutines.

Dynamical normal modes have been used to speed up, via invariants, the transport of two or more (possibly different) ions (Palmero et al., 2013, 2014; Lu et al., 2015) and to design trap expansions or compressions of ion chains in a common trap (Palmero, Martínez-Garaot, Alonso et al., 2015), ion separation (Palmero, Martínez-Garaot, Poschinger et al., 2015), and two-ion phase gates driven by spin-dependent forces (Palmero et al., 2017). However, for some operations a point transformation ${ }^{5}$ to define the dynamical modes does not exist.

For 2D systems the condition that an uncoupling point transformation exists is simply that the principal axes of the equipotential ellipses, see Fig. 9, do not rotate in the laboratory frame (Lizuain, Palmero, and Muga, 2017). If the ellipses are translated, expanded, or compressed, dynamical normal modes may be defined via point transformations. An operation for which the point transformation does not exist in

\footnotetext{
${ }^{5}$ In a point transformation new coordinates depend only on old ones.
} 
D. Guéry-Odelin et al:: Shortcuts to adiabaticity: Concepts, methods, ...

TABLE V. Experiments using STA methods. CD: counterdiabatic driving; IE: inverse engineering; OL: optical lattice; STIRAP: stimulated Raman adiabatic passage; NV: nitrogen vacancy; FAQUAD: fast quasiadiabatic dynamics.

\begin{tabular}{|c|c|c|c|}
\hline Reference & System & Operation & Method \\
\hline Couvert et al. (2008) & Ultracold atoms & Transport & Fourier-based IE \\
\hline Bason et al. (2012) & $\begin{array}{l}\text { Two-level Landau-Zener system } \\
\text { (BEC in OL) }\end{array}$ & Population inversion & $\mathrm{CD}+$ unitary transformation \\
\hline \multirow[t]{2}{*}{ Bowler et al. (2012) } & Trapped ions $(1,2)$ & Transport & Fourier transform \\
\hline & Trapped ion chain (9 ions) & Separation & FAQUAD \\
\hline Sun et al. (2012) & Overhead crane & Transport & Bang-bang \\
\hline Walther et al. (2012) & 1 and 2 ions & Transport & Optimized drivings \\
\hline Richerme et al. (2013) & 14 spins in linear trap (Ising model) & Adiabatic quantum simulation & Local adiabatic \\
\hline Zhang et al. (2013) & $\mathrm{N} V$ center in diamond & Assisted adiabatic passage & $\mathrm{CD}$ and accelerated $\mathrm{CD}$ \\
\hline Ruster et al. (2014) & 2 ions & Separation & $\begin{array}{l}\text { Implement a function } \\
\text { for equilibrium distance }\end{array}$ \\
\hline Kamsap et al. (2015) & Large ion clouds & Transport & Numerical simulations \\
\hline Rohringer et al. (2015) & 1D BEC & Expansion or compression & Scaling \\
\hline Alonso et al. (2016) & Trapped ion & Transport & Bang-bang \\
\hline Du et al. (2016) & Three-level Rb & STA-STIRAP & $\mathrm{CD}+$ unitary transform \\
\hline Martínez et al. (2016) & Brownian particle in optical potential & Equilibration & Engineered swift equilibration \\
\hline An et al. (2016) & Trapped ion & Simulated transport & $\begin{array}{l}\mathrm{CD} \text { and unitarily equivalent } \\
\text { protocols }\end{array}$ \\
\hline Navez et al. (2016) & $\begin{array}{l}\text { Atoms in state-dependent } \\
\text { "moving buckets" }\end{array}$ & Interferometer & Choose convenient timing \\
\hline Guo and Chu (2017) & Optical waveguides & Demultiplexing & Invariant-based IE \\
\hline Z. Zhang et al. (2017) & Superconducting phase qubit & Measure Berry phase & $\mathrm{CD}$ \\
\hline $\begin{array}{l}\text { Chupeau, Ciliberto } \\
\text { et al. (2018) }\end{array}$ & Brownian particle in quadratic potential & Control of temperature & Inverse engineering \\
\hline Cohn et al. (2018) & 2D array of 70 ions in Penning trap & Create highly entangled state & Bang-bang \\
\hline Deng, Diao et al. (2018) & Anisotropic Fermi gas & Expansion or compression & Scaling \\
\hline Kaufmann et al. (2018) & Trapped ion & Transport & Invariant-based IE \\
\hline Ness et al. (2018) & Ultracold $\mathrm{K}$ atoms in optical lattice & Transport & Invariants \\
\hline Smith et al. (2018) & Two-level in ${ }^{171} \mathrm{Yb}^{+}$ & Qubit rotations & $\mathrm{CD}$ \\
\hline Wang et al. (2018) & Superconducting Xmon qubit & Quantum gates & $\mathrm{CD}$ \\
\hline Hu et al. (2018) & Trapped ${ }^{171} \mathrm{Yb}^{+}$ion & Speed up Landau-Zener & Optimized-phase CD \\
\hline Z. Zhang et al. (2018) & Superconducting Xmon qubit & Measure work statistics & $\mathrm{CD}$ \\
\hline $\begin{array}{l}\text { Zhou, Jin, and } \\
\text { Schmiedmayer (2018) }\end{array}$ & $\mathrm{BEC}$ & Prepare BEC in bands of OL & Optimized pulse sequences \\
\hline Faure et al. (2018) & $\mathrm{RC}$ circuit & Change stationary regime & Inverse engineering \\
\hline Boyers et al. (2018) & $\mathrm{N} V$ center & Qubit population inversion & $\begin{array}{l}\text { Floquet engineering of } \\
\text { Hamiltonian components }\end{array}$ \\
\hline Kölbl et al. (2019) & $\mathrm{N} V$ center & Control of dressed states & $\mathrm{CD}$ \\
\hline T. Yan et al. (2019) & Xmon superconducting qutrit & Holonomic gates & $\mathrm{CD}$ \\
\hline $\begin{array}{l}\text { Vepsäläinen, Danilin, } \\
\text { and Paraoanu (2019) }\end{array}$ & Transmon & STA-STIRAP & $\mathrm{CD}$ \\
\hline Santos et al. (2019) & NMR of a Cloroform molecule & Single-qubit gates & $\mathrm{CD}$ driving \\
\hline
\end{tabular}

general is the separation of two different ions in an external potential $\alpha(t) x^{2}+\beta(t) x^{4}$, except when the ratio $\beta^{3}(t) / \alpha^{5}(t)$ is a constant. Similarly, for the rotation of a two-dimensional anisotropic trap holding one ion there is no point transformation leading to normal modes. A way out is to compensate for the inertial potential proportional to the angular momentum with an effective magnetic field. This is similar to the inertial force compensation with a homogeneous force in transport; see Sec. II.C.2.b. Finally, it is also possible to consider generalized transformations mixing coordinates and momenta (Lizuain et al., 2019).

\section{Ion transport}

Among the different operations on ions addressed by STA methods, ion transport is the most studied both theoretically (Palmero et al., 2013, 2014; Fürst et al., 2014; Lu, Muga et al.,
2014; Lu et al., 2015; Pedregosa-Gutierrez et al., 2015; Li, Zhang, and Chen, 2017; Lu, Ruschhaupt, and Muga, 2018; Tobalina, Alonso, and Muga, 2018) and experimentally in Paul traps (Bowler et al., 2012; Walther et al., 2012; Kamsap et al., 2015; Alonso et al., 2016; An et al., 2016; Kaufmann et al., 2018). The first two experiments were done simultaneously in Boulder (Bowler et al., 2012), using a Fourier transform technique, and Mainz (Walther et al., 2012), optimizing some driving protocols, to transport one or two ions diabatically for 300 to $400 \mu \mathrm{m}$ on a 5 to $10 \mu$ s timescale (a few oscillation periods) achieving final excitations below one motional quantum. Later, Kamsap et al. (2015) transported large ion clouds using numerical simulations to control the dynamics. In 2016, other shortcut techniques were used to improve transport experiments, for example, the bang-bang method using nanosecond switching of the trapping potentials by Alonso et al. (2016). The experiment by An et al. (2016) 


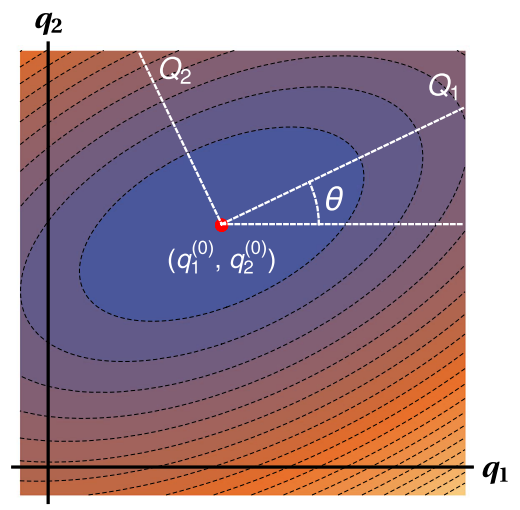

FIG. 9. Schematic representation of isopotential curves of a mass-weighted potential in the two-dimensional configuration space of laboratory-frame coordinates $q_{1}$ and $q_{2}$. These curves are ellipses centered at the moving equilibrium position $\left(q_{1}^{(0)}, q_{2}^{(0)}\right)$ with the orientation of the principal axes given by the angle $\theta$. The dynamical normal mode coordinates are $Q_{1}$ and $Q_{2}$. From Lizuain, Palmero, and Muga, 2017.

simulated CD-driven transport in an interaction picture with respect to the harmonic oscillation and also performed the compensating force approach as unitarily equivalent transport in the interaction picture. The driving forces were induced optically rather than by varying voltages of control electrodes. Finally Kaufmann et al. (2018) used invariant-based inverse engineering to design ion transport and measured internal infidelities less than $10^{-5}$, an important prerequisite for the success of quantum-information-processing schemes that rely on ion transport.

On the theoretical side, Fürst et al. (2014) used optimal control theory and the compensating force approach to analyze the transport of an ion in realistic conditions for state-of-the-art miniaturized ion traps. Given the simplicity of the approach and results, they considered the compensating force to be the method of choice for current experimental settings. Anharmonicity was found to play no significant role. This paper sets the relation between the desired trap trajectory and the voltages applied in the control electrodes. The model was later applied by Tobalina, Alonso, and Muga (2018) to analyze the energy cost of ion transport.

Lu, Muga et al. (2014) and Lu, Ruschhaupt, and Muga (2018) analyzed the effect of different colored noises on single-atom transport and how to mitigate their effect. Dynamical and static sensitivities are distinguished according to their dependence or independence with trap motion. They behave in opposite ways with respect to transport duration, which implies a transition between the dominance of the dynamical sensitivity at short times and of the static one at large times. The crossover is important as it demonstrates that the widespread expectation that shorter STA times are always more robust versus noise (a behavior that holds for the static but not for the dynamic sensitivity) is not necessarily true and that optimal times exist with respect to robustness. Li, Zhang, and Chen (2017) proposed trigonometric protocols that minimize the classical excitation due to anharmonicities within a perturbative approach.

Two-ion transport was addressed by Palmero et al. (2013, 2014). Also, Lu et al. (2015) designed optimal transport of two ions under slow spring-constant drifts. Designing fast transport of two different ions is challenging because the simple compensating force approach is not possible if only forces induced by the electrodes are applied. They depend only on the charges, whereas the compensating forces should depend on the mass (Palmero et al., 2014). Dynamical normal modes can however be defined so the problem is solved using invariants (Palmero et al., 2014). Two-ion transport may also be performed with "spin-dependent" optically induced forces that may be different for different internal states. An interesting application is the implementation of fast phase gates in which different phases are imprinted depending on the internal states because of the different motions induced. The invariant-based design of the ion trajectories guarantees a robust phase because of its geometric nature and its independence on the motional state (Palmero et al., 2017). Similar ideas may be applied to design a single-ion driven interferometer to measure unknown small forces (Martínez-Garaot, Rodriguez-Prieto, and Muga, 2018). Interferometry driven by STA trajectories (using ions or neutral atoms) (Navez et al., 2016; Martínez-Garaot, Rodriguez-Prieto, and Muga, 2018) offers, compared to the usual schemes where the systems evolve freely along separated branches, the possibility to control the timing and the sensitivity, the absence of wave packet dispersion, and robustness versus initial motional states.

\section{Other operations}

Fast ion separation.-Separating two ions, or more generally a chain, is a delicate operation and STA-enhanced experiments (Bowler et al., 2012; Ruster et al., 2014) provide excitations above one motional quantum per ion. Bowler et al. (2012) used a FAQUAD approach, and Ruster et al. (2014) optimized control parameters. In theoretical works (Home and Steane, 2005; Nizamani and Hensinger, 2012; Kaufmann et al., 2014; Palmero, Martínez-Garaot, Poschinger et al., 2015) the control process is modeled as a time-dependent evolution of the parameters in the external confinement potential $\alpha(t) x^{2}+\beta(t) x^{4}$. The difficulties come from the change of sign of $\alpha(t)$ from positive (in the initial trap) to negative values (to form the central barrier). When the harmonic confinement vanishes, the values of $\beta$ are bounded by experimental limitations. Thus the confinement becomes weak, levels get close to each other, and the ions suffer heating. If the ions are different, further difficulties arise as a consequence of the general absence of normal modes based on point transformations (Lizuain, Palmero, and Muga, 2017), as commented on previously; see Sec. III.A.1.

Ion expansions and compressions.-Palmero, MartínezGaraot, Alonso et al. (2015) analyzed by invariant-based inverse engineering how to expand or compress ion chains of equal or unequal ions, and Torrontegui et al. (2018) studied how to speed up a single-ion heat pump. This work analyzed the possibility to implement repulsive potentials. They are found to be feasible in the radial direction by turning of the radio-frequency drive and relying on the direct-current (cap) electrodes. Earnshaw's theorem does not allow for absolute minima so that a minimum in the axial direction of the trap corresponds to a maximum in the radial direction.

Penning traps. - Shortcuts have been applied as well to Penning traps: Kiely et al. (2015) designed via invariants a 


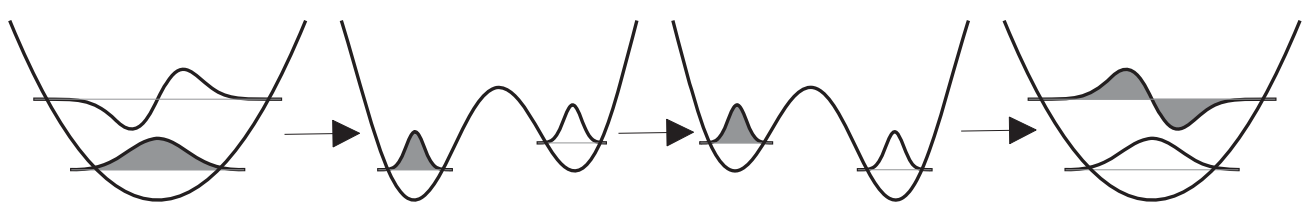

FIG. 10. Vibrational level inversion by STA processes based on trap deformations: demultiplexing, bias inversion, and multiplexing. From Martínez-Garaot et al., 2013.

nonadiabatic change of the magnetic field strength to change the radial spread without final excitations, and Cohn et al. (2018) implemented experimentally a protocol to produce entangled states in a Dicke model realized in a twodimensional array of trapped ions.

Rotations.-Palmero et al. (2016) provided invariant-based shortcuts to perform fast rotations of an ion in a 1D trap. ${ }^{6}$ Lizuain et al. (2019) designed fast rotations of a 2D anisotropic trap to produce a rotated version of an arbitrary initial state when the two normal frequencies are commensurate.

\section{B. Double wells}

A double well is a useful potential to test fundamental quantum physics and applications in interferometry or quantum and classical information processing. Shortcuts using invariants have been proposed for several operations involving double wells and motional states, for example, to split oneparticle wave functions in linear or nonlinear settings (Torrontegui, Martínez-Garaot et al., 2013; Martínez-Garaot et al., 2016), to speed up the cotunneling of two interacting bosons (Martínez-Garaot, Ruschhaupt et al., 2015), to split ion chains as discussed in Sec. III.A.3, or to model the erasure of a bit with a Brownian particle (Boyd et al., 2018). Applications involving many-body systems are reviewed in Sec. III.G. Other STA operations in double wells are vibrational-state inversion and multiplexing or demultiplexing.

Vibrational mode multiplexing is the spatial separation of the vibrational modes of a harmonic trap. For the first two modes the separation is done by smoothly transforming the harmonic trap into a biased double well. Martínez-Garaot et al. (2013) maped the STA process from a two-level model into a realizable potential in coordinate space designing the time dependence of two control parameters. A fast inversion of the double-well bias, so that the lower well becomes the upper one and vice versa, can be performed by noticing that the operation, in an independent-well regime, amounts to a transport process, so that the compensating force approach can be applied (Martínez-Garaot, Palmero et al., 2015) to design the time dependence of the bias. Combining sequentially multiplexing, bias inversion, and demultiplexing leads to a fast inversion of vibrational levels using only a transient trap deformation and no excited internal states; see Fig. 10. Interestingly, there is no adiabatic path connecting the ground and the excited states, but a combination of STA processes leads to the desired state. The explanation is that inverting the bias is in fact adiabatic within the approximation of

\footnotetext{
${ }^{6}$ Video in https://www.youtube.com/watch?v=ToQXnd_FdUw.
}

independent wells, in other words, during a timescale which is short compared to the duration of the process.

Bücker et al. (2011, 2013) independently performed motional-state inversions by shaking anharmonic potentials guided by an OCT algorithm to produce twin atom beams and interferometry applications, but a smooth STA-based deformation similar to Fig. 10 has not yet been implemented experimentally.

\section{Cavity quantum electrodynamics}

Entangled state preparation in cavity quantum electrodynamics. - In cavity quantum electrodynamics atoms and light are confined such that the quantum states of the atom are protected by allowing only controllable transitions compatible with the modes of the cavity and thus isolating the atom from any electromagnetic environment other than the cavity itself. These systems have recently opened up new prospects to implement large-scale quantum computation and to generate nonclassical states.

An early proposal to produce entangled states faster than adiabatically by moving atoms in and out of an optical cavity was provided by Marr, Beige, and Rempe (2003): the proposal was to use cavity leakage to stabilize the desired adiabaticlike time evolution by damping away population in unwanted states. However, a consequence is that the success rate decreases.

In the post-2010 era the use of shortcuts to inverse engineer the dynamics of two atoms inside a cavity was first discussed by Lu, Xia, Shen, Song, and An (2014). They used the counterdiabatic driving formalism to create maximal entanglement between the two atoms but, due to the complexity of the resulting counterdiabatic Hamiltonian, they had to introduce an alternative, physically feasible Hamiltonian that needs auxiliary internal levels, an extra laser field, and an extra cavity mode. The same group tried a different strategy using invariants to accelerate the state transfer between two threelevel atoms in a cavity quantum electrodynamics (QED) system (Lu, Xia, Shen, and Song, 2014).

Y.-H. Chen et al. (2014a) used invariants combined quantum Zeno dynamics (QZD) to simplify the dynamics and speed up the population transfer between two atoms trapped in a cavity. The quantum Zeno effect inhibits transitions by frequent measurements so that the system evolves in the so-called Zeno subspace (Facchi and Pascazio, 2002). This quantum Zeno dynamics can also be achieved via a strong continuous coupling (Facchi and Pascazio, 2002). In particular, Muga et al. (2008) discussed the mapping between discrete and continuous interactions. Following Y.-H. Chen et al. (2014a), many other papers have used the QZD approximation to decouple different Hilbert 
subspaces by assuming that the atom-cavity coupling is much larger than the driving field Rabi frequencies. The simplified effective Hamiltonians were controlled with the use of different STA techniques to design fast and robust protocols against decoherence produced by atomic spontaneous emission and cavity leakage; see, for example, Wu, Ji, and Zhang (2017b). Chen et al. (2014b) extended the idea of combining QZD and STA methods to control the dynamics of atoms trapped in distant cavities connected through a fiber.

A requirement for the success of quantum mechanics in information processing tasks is scalability toward multiparticle $(N>2)$ setups. The macroscopic character of cavity QED setups favors the interaction among qubits and thus scalability. Numerous setups and control designs have been proposed to generate large entangled states using STA methods and the QZD assumption, in particular, large- $N$ entangled $W$ states (Huang, Zhong, and Chen, 2015; Chen, Huang et al., 2016; Huang, Chen, and Wang, 2016b; Kang et al., 2016a, 2016b; Song and Chen, 2016; Z. Wang et al., 2016; Yu et al., 2017; Yang et al., 2018), or GreenbergerHorne-Zeilinger (GHZ) states (Chen, Xia, Song, and Chen, 2015; Huang et al., 2016; Huang, Chen, and Wang, 2016a; Shan et al., 2016; Ye et al., 2016; Xu et al., 2017; X. Zhang et al., 2017). Moreover, using the QZD scheme several presented various STA setups to create 3D entanglement between atoms individually trapped in distant optical cavities connected by a fiber (Liang, Su et al., 2015; Lin et al., 2016; Wu, Ji, and Zhang, 2016; Wu, Song et al., 2016) or between two atoms trapped in a single cavity (He et al., 2016; Yang et al., 2017).

Shortcuts have also been proposed to generate other less common entangled states, such as large NOON states of two sets of $\Lambda$ atoms in distant cavities using invariants (C. Song et al., 2016), and, using CD driving, a three-atom singlet in a common cavity (Z. Chen et al., 2016), tree-type 3D entangled states (Wu, Ji, and Zhang, 2016; Wu, Su, Ji, and Zhang, 2017), two-atom qutrit entanglement (Peng et al., 2017), or maximally entangled states of two Rydberg atoms (Zhao et al., 2017).

Other applications.-Different STA-enhanced quantum gates were designed within the QZD condition. For example, invariant-based inverse engineering was used to develop Toffoli gates (Song, Xia, and Song, 2014), phase gates (Chen, Xia, Chen, and Song, 2015; Liang, Wu et al., 2015), CNOT gates (Liang, Song et al., 2015), or swap gates (Liang, Ji et al., 2015). Wu, Ji, and Zhang (2017a) used the dressed-state method (Baksic, Ribeiro, and Clerk, 2016) to design a fast CNOT gate in a cavity QED system which consists of two identical five-level atoms in two single-mode optical cavities connected by a fiber.

Shortcuts have been proposed as well to produce single photons on demand in an atom-cavity system approximated by three levels (Shi and Wei, 2015). A system of distant nodes in two-dimensional networks (cavities with a $\Lambda$-type atom) was approximated by a three-level $\Lambda$ system in Zhong (2016) and then STA techniques were applied to achieve fast information transfer.

Optomechanical systems. - Shortcuts have been applied as well to optomechanical systems. One of the early applications of the invariant-based approach by Chen, Ruschhaupt et al. (2010) was to cool down a mechanical resonator in a cavity optomechanical system with external optical fields ( $\mathrm{Li}$, $\mathrm{Wu}$, and Wang, 2011). ${ }^{7}$ For more recent applications, see X. Zhou et al. (2017), Chen, Shi et al. (2018), and H. Zhang et al. (2018).

\section{Superconducting circuits}

Superconducting circuits have recently made rapid progress and become a leading architecture for quantum technologies (Wendin, 2017). In this context STA techniques were independently developed under the names "derivative removal by adiabatic gate" for single qubits (Motzoi et al., 2009) and weak anharmonicity with average Hamiltonian (Schutjens et al., 2013) for multiqubit setups and multilevel systems (qutrits); see also Theis, Motzoi, and Wilhelm (2016), Lu et al. (2017), and Theis et al. (2018) and the discussion in Sec. II.B.1, focusing on avoiding unwanted transitions to spectrally neighboring energy levels.

Other techniques for sped-up manipulations take into account the peculiarities of the experimental settings, such as small nonlinearities in the qubits (implying that unwanted transitions are not necessarily off resonant), or a need for smooth pulses (versus square pulses common in nuclear magnetic resonance). In particular, Martinis and Geller (2014) considered a two-level model where only the $\sigma_{z}$ term changes to achieve fast gates with the $\sigma_{x}$ term constant in $H=H_{x} \sigma_{x}+H_{z} \sigma_{z}$. Relating the error in the gate operation to the Fourier transform of the properly scaled rate of change of the polar angle $\theta=\arctan \left(H_{x} / H_{z}\right)$, optimal protocols were found, minimizing the integrated error for any time larger than some chosen time.

Other works apply the standard STA methods, in particular, CD driving, to transfer information between distant nodes of flux qubits in annular and radial superconducting networks (Kang, Shi et al., 2017), to complete Bell-state analysis for two superconducting-quantum-interference-device qubits (Kang, Chen et al., 2017), or to measure the Berry phase (Z. Zhang et al., 2017) in a phase qubit.

Further applications of CD shortcuts include the proposal or realization of gates. Zhang et al. (2015) proposed holonomic one- and two-qubit gates based on four-level systems in superconducting transmons. Experiments were performed to implement single-qubit quantum gates in a superconducting Xmon system (Wang et al., 2018) with a hybrid CD + DRAG approach, and non-Abelian geometric gates with a ladder three-level system (T. Yan et al., 2019). Superconductors also play a role as a possible platform to realize topological quantum information processing based on "braiding" nonAbelian quasiparticles. Karzig et al. (2015) explored CD protocols to realize these braiding operations in finite time.

Interfacing different architectures to make better use of their optimal features may be important to jump from proof of principle to practical technologies. In particular, superconducting circuits may be combined with optomechanical systems producing hybrid quantum systems. X. Zhou et al. (2017) proposed a protocol to efficiently convert microwave to

\footnotetext{
${ }^{7}$ Zhang, Li, and Feng (2013) proposed instead electromechanical cooling.
} 
optical photons, enabling the transmission of information through optical fibers with minimal loss. One more application of shortcuts in superconducting circuits is to create photonic cat states. Stored in high- $Q$ resonators these states could lead to efficient universal quantum computing (Puri, Boutin, and Blais, 2017).

\section{E. Spin-orbit coupling}

Discrete models. - Coherent spin manipulation in quantum dots via electric, magnetic, and spin-orbit coupling control is one of the avenues to solid-state based quantum information. STA methods have been proposed to speed up operations and fight decoherence also in this context. In particular, STA are welcome to speed up double quantum dots with SOC control, since strong fields nontrivially slow down the operations involving spin-orbit coupling and tunneling (Khomitsky, Gulyaev, and Sherman, 2012).

The interplay between motional and spin degrees of freedom is typically modeled by an effective discrete Hamiltonian where STA techniques are applied. The physics behind the model, however, makes the exercise nontrivial, as the different matrix elements are not always controllable independently by the available external manipulations. An example of these physical constraints and a way out was provided by Ban et al. (2012a, 2012b), in which the spin dynamics in a quantum dot with spin-orbit coupling and a weak magnetic field is controlled by time-dependent external electric fields only. The dependence of the effective $2 \times 2$ Hamiltonian on the electric field makes a $\mathrm{CD}$ approach nonviable with electric control. However, invariant-based inverse engineering is applicable and circumvents the difficulty.

Two electrons in a double dot offer more freedom since different electric fields can be applied to each dot. Thus CD driving combined with a unitary transformation could be applied to induce fast singlet-triplet transitions (Ban and Chen, 2014), again within a $2 \times 2$ Hamiltonian modeling.

Synthetic spin-orbit coupling in ultracold atoms and condensates is also of interest to control internal and motional states. Invariant-based protocols to simultaneously control the internal (related to its pseudospin-1/2) and motional states of a spin-orbit-coupled BEC in Morse potential have also been studied by Ban et al. (2015) by a $2 \times 2$ effective Hamiltonian.

A $4 \times 4$ Hamiltonian was used in $\mathrm{Li}$, Chen et al. (2018) to model and design the transport of a qubit encoded in the electron spin among two quantum dots, performing simultaneously an arbitrary qubit rotation (gate). The transfer may be extended sequentially to a chain of dots. These processes need time-dependent control of the spin-orbit and interdot tunneling couplings. The dynamical engineering of the four-level system applies the technique developed by $\mathrm{Li}$, MartínezCercós et al. (2018) based on the geometry of 4D rotations.

Models with a continuum.-Models that retain motional (1D, in $x$ direction) and internal degrees of freedom of the electron without discretization began with Čadež, Jefferson, and Ramšak (2013), who considered an electron with spinorbit coupling in a moving harmonic quantum dot,

$$
H(t)=\frac{p_{x}^{2}}{2 m^{*}}+\frac{m^{*} \omega^{2}}{2}[x-\xi(t)]^{2}+p_{x}\left(\alpha \sigma_{y}-\beta \sigma_{x}\right),
$$

where $m^{*}$ is the effective electron mass, $\xi(t)$ is the time-dependent position of the harmonic trap, and $\alpha$ and $\beta$ are Rashba and Dresselhaus spin-orbit couplings. The dynamics of this model is exactly solvable via invariants, and fast bang-bang trap trajectories leading to spin control (e.g., spin flip) without final excitation were found for $\beta=0$. In Čadež, Jefferson, and Ramšak (2014), $\beta$ is fixed to zero while $\alpha$ remains time dependent. The dynamics can still be found exactly and expressions are given for dynamical and geometrical phases in closed-loop trajectories of the control parameters. Chen, Jiang et al. (2018) translated these ideas to synthetic spin-orbit couplings in BECs and applied inverse engineering of $\xi(t)$ and $\alpha(t)$ $(\beta=0)$ from auxiliary Newton-like equations for the centerof-mass position of the condensate and its spin precession. Again spin is flipped fast by proper design of the controls.

Controllable linear-in-momentum interactions to implement direct (not unitarily transformed) CD-driven transport in the lab frame, i.e., with the form $H_{C D}=p_{x} \alpha(t)$, with $\alpha=\dot{q}_{c}$, see Torrontegui et al. (2011) and Sec. II.C.2.b, may in principle be implemented by synthetic spin-orbit coupling for one of the spin components (Tobalina, Alonso, and Muga, 2018). Note that the change of sign for the other component is crucial to determine the possible applications. This dependence precludes, for example, transporting a qubit, but it is useful to set different paths in interferometry (MartínezGaraot, Rodriguez-Prieto, and Muga, 2018).

\section{F. Nitrogen-vacancy centers}

Quantum information processing with $\mathrm{N} V$ centers is appealing because of the possibility to operate with qubits at room temperature. Yet, decoherence is still a problem and several STA protocols have been implemented in experiments or proposed theoretically.

The counterdiabatic driving approach was applied to design "universal" sets of fast and robust non-Abelian geometric gates: X.-K. Song, Zhang, Ai et al. (2016) proposed to use four $\mathrm{N} V$ centers around a whispering-gallery mode microsphere cavity, while Liang et al. (2016) made use of a single $\mathrm{N} V$ center coupled to a ${ }^{13} \mathrm{C}$ nuclear spin both modeled as twolevel systems. Du et al. (2017) proposed a three-level scheme for the $\mathrm{N} V$ center where two of the couplings are due to microwave fields, and the third coupling is mechanically induced.

Experiments have shown the flexibility of $\mathrm{N} V$ centers to implement STA protocols for discrete systems: Kleißler, Lazariev, and Arroyo-Camejo (2018) implemented onequbit holonomic gates proposed by Liang et al. (2016) in an effective two-level system driven by a microwave field with controllable, time-dependent detuning, Rabi frequency, and phase; Liu et al. (2018) used the eigenbasis of the dynamical invariant $I(t)$ associated with $H(t)$ as the auxiliary basis for constructing geometrical gates; and in B. B. Zhou et al. (2017), stimulated Raman adiabatic passage in a three-level system was sped up using dressed-state driving in terms of the original controls of the reference Hamiltonian. 
The possibility of physically implementing two microwave couplings and one mechanical coupling among three levels is one of the interesting features of STA applied to NV centers (Amezcua, Golter, and Wang, 2017; Du et al., 2017). Thanks to this structure Kölbl et al. (2019) used CD protocols experimentally to implement the initialization, readout, and coherent control of three-level dressed states. These states offer efficient coherence protection, better than the one achieved by two-level systems.

\section{G. Many-body and spin-chain models}

Many-body systems display controllable emergent properties and phenomena potentially useful in metrology and quantum simulation or computation, as well as in applications such as quantum light generation, memory devices, or precise sensing and communications. Slowly varying control parameters is one of the key tools to prepare and manipulate quantum many-body systems. In particular, adiabaticity plays a central role in understanding or implementing the quantum Hall effect, topological insulators, adiabatic computing, certain quantum phases [see Bachmann, De Roeck, and Fraas (2017) and references therein], or to maximize entanglement (Dorner et al., 2003).

This discussion sets a strong motivation for developing STA approaches in many-body systems. In some cases "exact" shortcuts may be found, for example, after having applied mean-field theories or semiclassical approximations for a large number of particles $N$, or due to exact solvability, as for self-similar dynamics for specific interactions (Muga et al., 2009; del Campo, 2011, 2013; del Campo and Boshier, 2012; Deffner, Jarzynski, and del Campo, 2014). Rohringer et al. (2015) demonstrated scaling behavior and shortcuts for expansions and compressions of phase-fluctuating quasi-1D Bose gases and more recently Deng, Chenu et al. (2018) and Deng, Diao et al. (2018) in a three-dimensional anisotropic "unitary Fermi gas."

Indeed, early STA experiments showed that manybody systems may benefit from STA techniques: expansions of clouds of cold thermal atoms were handled via invariants in the independent-atom approximation (Schaff et al., 2010), and interacting Bose-Einstein condensates could be expanded fast using scaling in the mean-field approximation (Schaff, Capuzzi et al., 2011; Schaff, Song et al., 2011).

However, adiabaticity is often problematic for many-body systems making STA challenging. The difficulties are illustrated by the "orthogonality catastrophe": two ground states for two slightly different values of a control parameter $\lambda$ may become orthogonal in the thermodynamic limit so that "strict" adiabaticity breaks down in essentially zero time. For certain drivings this occurs even with a finite gap. Milder definitions of adiabaticity are possible (Bachmann, De Roeck, and Fraas, 2017) referring to local variables rather than to the global $N$-body wave function.

The difficulties to implement adiabatic drivings are also evident in phase transitions across a quantum critical point, which lead to excitations for any finite crossing rate of the parameter. CD drivings can be found for the family of models which are solvable by a transformation into independent fermions, as for the 1D Ising model in a traverse field (del Campo, Rams, and Zurek, 2012), ${ }^{8}$

$$
H=-\sum_{n=1}^{N}\left(\sigma_{n}^{x} \sigma_{n+1}^{x}+g \sigma_{n}^{z}\right) .
$$

After a Jordan-Wigner transformation, the Hamiltonian is decomposed into a series of independent Landau-Zener Hamiltonians for which the counterdiabatic driving is known. However, in spin space $H_{C D}$ is highly nonlocal; it involves long-range, multibody interactions, and is increasingly important and divergent near the critical point (del Campo, Rams, and Zurek, 2012; del Campo, 2013; Damski, 2014, 2015; del Campo and Sengupta, 2015; Duncan and del Campo, 2018). For specific models the nonlocality may be circumvented. Thus Okuyama and Takahashi (2016) achieved a local CD for the Toda lattice using the machinery of Lax pairs, and Takahashi (2013b) for the $X Y$ model used a "fixed-point condition" where $H_{C D}=0$.

Systematic efforts have been done to find approximate shortcuts based on truncation of the CD terms (del Campo, Rams, and Zurek, 2012; Damski, 2014) by restricting the range of the interaction, imposing adiabaticity only locally via local interactions (Mukherjee, Montangero, and Fazio, 2016), or optimizing an approximate Ansatz for $H_{C D}$ based on simple (in particular two-body) auxiliary fields. This optimization requires spectral and wave function knowledge that is available for finite systems of experimental interest (Saberi et al., 2014). Such detailed information is however typically not available for larger systems so another major research thread is to avoid using explicit spectral information to construct the shortcut. A variational approach proposed by Sels and Polkovnikov (2017), see Sec. II.B.2, moved in that direction. In particular, the aim of adiabatic computing is to find the ground state, which encodes the solution of a computational problem, by adiabatic following, precisely because it cannot be calculated. Yoshimura and Freericks (2015) proposed to apply a method to estimate the probability to be in the ground state from time-dependent measurements without knowing the Hamiltonian or its eigenfunctions, in order to optimize approximate shortcuts. Another phenomenological way out for some applications may be to optimize control parameters experimentally. For example, Cohn et al. (2018) proposed an optimization of a bang-bang protocol for the external parameter to produce entangled states in a Dicke model realized in a two-dimensional array of trapped ions in a Penning trap.

Josephson junctions and Lipkin-Meshkov-Glick-like models.-Much work has been done to apply shortcuts to many-body models which are either similar to or particular cases of the Lipkin-Meshkov-Glick (LMG) model

$$
H_{\mathrm{LMG}}=\hbar\left[\Omega J_{z}+W\left(J_{x}^{2}+J_{y}^{2}\right)+V\left(J_{x}^{2}-J_{y}^{2}\right)\right],
$$

\footnotetext{
${ }^{8}$ Another solvable model, where the $\mathrm{CD}$ driving involves as well many-body interactions, is the one-dimensional Kitaev honeycomb model (Kyaw and Kwek, 2018). It was proposed to generate highly entangled "cluster states" needed to implement "measured based quantum computation."
} 
where

$$
\begin{aligned}
& J_{x}=\frac{1}{2}\left(a^{\dagger} b+a b^{\dagger}\right), \\
& J_{y}=\frac{1}{2 i}\left(a^{\dagger} b-a b^{\dagger}\right),
\end{aligned}
$$

and

$$
J_{z}=\frac{1}{2}\left(a^{\dagger} a-b^{\dagger} b\right)
$$

are "pseudospin" operators, with $a$ and $b$ being annihilation operators for two sites or two internal states. The same type of Hamiltonian appears in spin models where the operators $J_{\alpha}$ are to be interpreted as global angular momentum operators, usually denoted instead by $S_{\alpha}=(1 / 2) \sum_{i} \sigma_{\alpha}^{i}, \alpha=x, y$, and $z$, where $\sigma^{i}$ is the Pauli spin operator for site $i$. Table III depicts some of the original Hamiltonians for which shortcuts were developed.

Bosonic Josephson junctions were treated by Juliá-Díaz et al. (2012) to generate spin-squeezed states. The junction was modeled with a two-site Bose-Hubbard Hamiltonian; see Table III. The ground state for $U>0$ (antiferromagnetic LMG model) is unique. In the large- $N$ limit and in the Fock basis of boson imbalance between the two wells the system may be treated semiclassically, i.e., considering a continuous rather than discrete population imbalance, for time dependent $U(t)$ and fixed $J$. The model simplifies to a single particle in an oscillator with time-dependence frequency. Invariants can then be used to design shortcuts and produce spin-squeezed states. A similar approach was later applied when the time dependence is in $J$, which is better suited for "internal junctions" where the connected states are not at different locations but in different internal states (Yuste et al., 2013).

Other works have treated the two-site Bose-Hubbard Hamiltonian for low particle numbers, specifically $N=2$ : Opatrný and Mølmer (2014) proposed it as a toy model for a transition between a superfluid (maximally delocalized) state and a Mott-insulator ground state and explained the difficulties to physically implement the CD Hamiltonian ${ }^{9}$; MartínezGaraot et al. (2014) applied Lie-algebraic methods to this problem, see Sec. II.B.2.a, to get STA without experimentally unfeasible generators; and Stefanatos and Paspalakis (2018b) optimized shortcuts via optimal control to maximize entanglement.

\footnotetext{
${ }^{9}$ Suppose that $A$ and $B$ are feasible operators in same $H_{0}$ and that $H_{C D}$ needs $[A, B]$, which is difficult to implement directly. According to the Baker-Hausdorff-Campbell formula, applying the sequence of operators $b B \rightarrow a A \rightarrow-b B \rightarrow-a A$, where $a$ and $b$ are coefficients, during time intervals $\Delta t$ amounts to applying $i a b[A, B] \Delta t$ for a time $\Delta t, \quad e^{i a A \Delta t} e^{i b B \Delta t} e^{-i a A \Delta t} e^{-i b B \Delta t}=e^{-a b[A, B] \Delta t^{2}}+O\left(\Delta t^{3}\right)$. Thus, in principle implementing $a b \Delta t[A, B]$ is possible with $\pm a A$ and $\pm b B$, but the problem lies in the scaling of the coefficients with $\Delta t$. For a given target value of $c=a b \Delta t$, then $a b=c / \Delta t$, which leads to intense pulses. If high intensities are available the dynamics with $H_{0}$ becomes more adiabatic so that the $\mathrm{CD}$ term is not really needed (Opatrný and Mølmer, 2014).
}

Opatrný et al. (2016) looked for maximally spin-squeezed "Dicke states" for finite $N$ approximating the CD terms as in Opatrný and Mølmer (2014) and discussed how to implement compensating terms that go beyond quadratic order in the collective spin operators by means of suitable time sequences switching between quadratic operators.

The negative nonlinear coupling coefficient (ferromagnetic model) was studied by Takahashi (2013b) by providing the $\mathrm{CD}$ term for the ground state in the large- $N$ thermodynamic limit. It generally diverges at the critical point. However, protocols satisfying a "fixed-point" condition such that $H_{C D}=0$ were shown to be feasible in some instances. Campbell et al. (2015) used approximate CD terms identified by retaining dominant few-body terms, that may be optimized, rather than calculated from spectral information, with the aid of the instantaneous ground state. By applying a small longitudinal field that avoids the critical point, a mean-field prescription was applied using invariant-based engineering by Takahashi (2017b), and counterdiabatic driving by Hatomura (2017). Hatomura (2018) applied the semiclassical large- $N$ approximation to generate a CD term, which, corrected by finite-size terms, avoids divergence at the critical point to generate cat states. Formally the process amounts to performing a transition from a single to a double well assisted by CD driving. The effect of particle losses was studied by Hatomura and Pawłowski (2019).

State transfer.-Fast quantum state transfer in linear chains was also theoretically addressed with STA concepts and techniques. Agundez et al. (2017) used a CD approach plus unitary transformation in a spin chain and Z.-M. Wang et al. (2016) sped up a slow protocol by scaling up the Hamiltonian. A similar method was applied by Ren, Wang, and $\mathrm{Gu}$ (2017) to cut a chain into two pieces. Ban et al. (2019) proposed to inverse engineer the tunneling barriers to transfer two-electron entangled states from one edge of an array of quantum dots described by the Hubbard model to the other. Finally, Longhi (2017) proposed a nonadiabatic fast protocol of robust excitation transfer in a non-Hermitian Hatano-Nelson tight-binding linear chain assisted by gain and loss gradients to cancel nonadiabatic transitions providing a fast state transfer in coupled-resonator optical waveguide structures.

ID Tonks-Girardeau gases and fermionic systems.-Under 1D effective confinement, the control and dynamics of noninteracting fermions or Tonks-Girardeau gases can be studied with solvable models that ultimately rely on the Slater determinant built with orbitals for single particle dynamics. Shortcuts have been applied to them in several works with different aims: Martínez-Garaot, Ruschhaupt et al. (2015) with the aid of the FAQUAD approach and Schloss et al. (2016) using invariants devised protocols to prepare macroscopic entangled states (NOON states) stirring a TonksGirardeau gas on a ring. The observation that leaking between two occupied states does not influence the fidelity of the gas, and that only leaks into modes above the Fermi level do, was systematically exploited and developed by Dowdall et al. (2017) for more general objectives to control fermionic systems. 


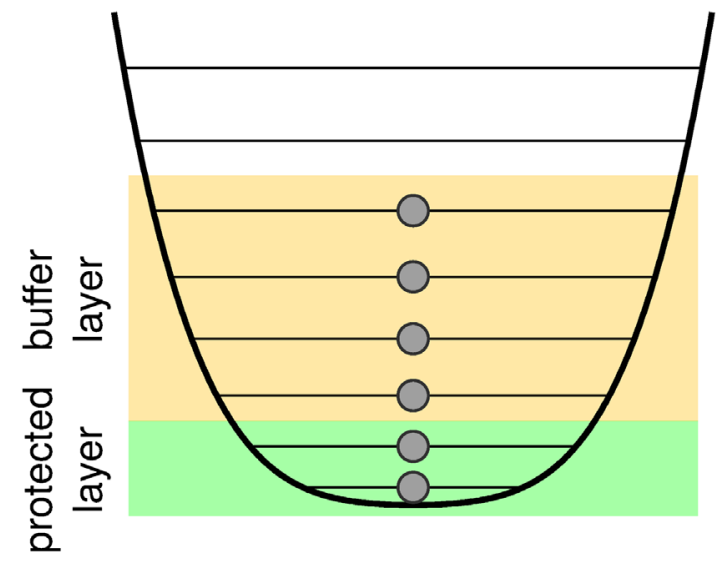

FIG. 11. To prevent transitions from the protected layer to higher-lying states during changes of the external parameters, a buffer layer of fermions is added. The Pauli principle prevents the protected fermions from accessing any level in the buffer zone. Adapted from Dowdall et al., 2017.

Dowdall et al. (2017) proposed to use Pauli blocking for speeding up adiabatic evolution of the ground state of a number of "protected fermions" by using an additional layer of buffer particles; see Fig. 11. The protected fermions cannot make a transition to higher-lying excited states because these are occupied, and the fidelity for the final state of the protected particles increases with the number of buffer particles.

\section{H. Metrology}

Quantum systems offer, via interference, entanglement, and squeezing, many interesting possibilities for metrological applications and for improving measurement sensitivities. Allowing for time dependence in the Hamiltonian can only enhance the possibilities to find useful protocols (Pang and Jordan, 2017) so shortcuts to adiabaticity will also play an important role in metrology. We have already seen throughout the review several examples: In Sec. III.A we mentioned how STA-enhanced interferometry, in which the states are STA driven along the interferometer branches, can increase sensitivities and decrease decoherence effects. Also, Secs. III.C, III.D, and III.G provide examples where shortcuts are used to create in different platforms states of metrological interest such as spin-squeezed states, NOON states, and other entangled states.

Here we underline the strong link between CD driving and optimal protocols for quantum metrology. Pang and Jordan (2017) pointed out that fundamental precision limits valid for time-independent Hamiltonians can be dramatically violated with appropriate time-dependent control. In particular, the Fisher information, whose inverse square root limits the precision to measure some parameter $g$ in the Hamiltonian $H_{g}$ for arbitrary estimation strategies, can beat the limits valid for time-independent Hamiltonians. The information on $g$ is transferred to a time evolution of a state measured at some time $T$. The upper bound for the Fisher information can be realized for superposition states whose components evolve at all times along the maximal and minimal eigenvalues of $\partial_{g} H_{g}(t)$. Here a slight modification of CD driving comes in handy. Similar to Sec. II.B, a Hamiltonian term $H_{c}(t)$ is added to $H_{g}(t)$ so that the dynamics follows exactly the eigenstates $\left|\psi_{k}(t)\right\rangle$ of $\partial_{g} H_{g}(t)$ [rather than the eigenstates of a zeroth Hamiltonian $H_{0}(t)$ as in regular CD driving]. Allowing for a freedom to choose phases $\theta(t)$ for each mode $H_{\text {tot }}=H_{g}(t)+H_{c}(t)$ may be written as

$H_{\mathrm{tot}}=-\sum\left|\psi_{k}(t)\right\rangle \dot{\theta}(t)\left\langle\psi_{k}(t)\left|+i \hbar \sum\right| \partial_{t} \psi_{k}(t)\right\rangle\left\langle\psi_{k}(t)\right|$,

with exactly the same form as Eqs. (18) and (19), except for the meaning of the wave functions. The way to find $g$ is in fact iterative, assuming first an estimate for $g$ used to improve the estimate and so on. All this opens an exciting avenue since the possible difficulties to implement counterdiabatic driving terms as well as the arsenal of remedies described in Sec. II.B are applicable.

\section{THE ENERGY COST OF STA, ENGINES, AND THE THIRD LAW}

\section{A. Energy costs}

In human affairs, shortcuts to reach a place or a goal may cost money or consume energy, although there is no universal recipe or law on the costs applicable to all circumstances. Some shortcuts are really for free, or almost, so they save time and resources, but others need a toll. Finding the "energy cost" of shortcuts to adiabaticity is a relevant and indeed, lately, popular goal. It is tempting to consider shortcuts to be energetically a free lunch since, by definition, the final energy is the same as for a slow adiabatic process. Many works, however, put forward different associated costs and imply that STA driving can hardly be free. In general the term "cost" has been used in a loose and heuristic way, without a fundamental analysis that justifies its suitability as an energy consumption. Actually most studies "define" rather than "find" the cost so that different definitions of cost given so far are not necessarily in conflict. As long as we put aside the propriety of the term, we may regard them as different aspects of the system energies or interactions, such as their evolution (transient values or time averages), excitations, fluctuations and flows, "intensities," or inequalities for several times and energies involved.

In an early study on the expansion and compression of a particle in a time-dependent harmonic oscillator (Chen and Muga, 2010), the cost was related to the time average of the particle energy. This average and its scaling with process time $t_{f}$ depend strongly on the STA applied. If the shortcut makes use of a properly chosen $\omega(t)$ (e.g., designed via invariants) and no extra Hamiltonian terms, it was found by Euler-Lagrange optimization that for the $n$th eigenstate the time-averaged energy obeys $\bar{E}_{n}>(2 n+1) \hbar /\left(2 \omega_{f} t_{f}^{2}\right)$ for $\left(\omega_{0} / \omega_{f}\right)^{1 / 2} \gg 1$ and $t_{f} \ll\left(\omega_{0} \omega_{f}\right)^{-1 / 2}$, where $\omega_{0}$ and $\omega_{f}$ are initial and final (angular) trap frequencies. Realizing this bound is indeed possible but at the price of infinite instantaneous power at boundary times (Cui, Chen, and Muga, 2016). The bound is relevant even in anharmonic traps since the trap depth $D$ should be larger than $\bar{E}_{n}$, which sets a scaling 
$t_{f} \gtrsim\left(\hbar / \omega_{f} D\right)^{1 / 2}$ for the minimal process time. ${ }^{10}$ The time average of the standard deviation of the energy was found to scale also as $t_{f}^{-2}$, tighter than the bound $\gtrsim t_{f}^{-1}$ that follows from the Anandan-Aharonov (AA) relation (Anandan and Aharonov, 1990),

$$
\tau \geq \frac{\hbar}{\overline{\Delta E}} \mathcal{L}
$$

setting $\tau=t_{f}$, where

$$
\begin{gathered}
\mathcal{L}=\arccos |\langle\psi(0) \mid \psi(\tau)\rangle|, \\
\overline{\Delta E}=\frac{1}{\tau} \int_{0}^{\tau}\left(\left\langle H^{2}\right\rangle-\langle H\rangle^{2}\right)^{1 / 2} d t .
\end{gathered}
$$

This is one of the first applications of "quantum speed limits" to shortcuts to adiabaticity, ${ }^{11}$ see Deffner and Campbell (2017). Many other applications have followed (Santos and Sarandy, 2015; Abah and Lutz, 2017; Campbell and Deffner, 2017) using AA and/or a Margoulis-Levitin-type of relation (Deffner and Lutz, 2013b). Note that a naive extension of the AA relation substituting $\overline{\Delta E}$ by the time-averaged energy $\langle H(t)\rangle$ is not valid in general for time-dependent Hamiltonians. Instead, a valid Margoulis-Levitin-type relation is (Deffner and Lutz, 2013a; Santos and Sarandy, 2015)

$$
\tau \geq \hbar \frac{|\cos \mathcal{L}-1|}{(1 / \tau) \int_{0}^{\tau}|\langle\psi(0)|H| \psi(t)\rangle|} .
$$

As a more recent example of other definitions, the average power computed as the total work divided by the process time was defined as the cost in Herrera et al. (2014). The cost has also been linked to the accumulated (Zheng et al., 2016) or time-averaged (Santos and Sarandy, 2015, 2018; Coulamy et al., 2016; Santos, Silva, and Sarandy, 2016) Frobenius norm of the Hamiltonian $\|H(t)\|=\sqrt{\operatorname{tr}\left[H(t)^{2}\right]}$ or of some $n$th power depending on the setting. This norm does not exist for many commonly found Hamiltonians such as the one for the harmonic oscillator. If it exists and $H=H_{0}+H_{C D}$, the time average for $H$,

$$
\Sigma_{H}\left(t_{f}\right)=\frac{1}{t_{f}} \int_{0}^{t_{f}} \sqrt{\operatorname{tr}\left[H_{0}^{2}+H_{C D}^{2}\right]} d t
$$

is larger than the one for the reference protocol

$$
\Sigma_{0}\left(t_{f}\right)=\frac{1}{t_{f}} \int_{0}^{t_{f}} \sqrt{\operatorname{tr}\left[H_{0}^{2}\right]} d t,
$$

\footnotetext{
${ }^{10}$ The energy in $\mathrm{CD}$ driving scales differently. For example, choosing a linear ramp for the reference $\omega(t), \bar{E}_{n}$ is independent of $t_{f}$, whereas the time-averaged variance [for all monotonous $\omega(t)$ ] goes as $t_{f}^{-1}$.

${ }^{11}$ The AA relation was later renamed a "Mandelstam-Tamm-type" relation, even if Mandelstam and Tamm did not consider timedependent Hamiltonians (Mandelstam and Tamm, 1945).
}

which suggests that $\mathrm{CD}$ driving always implies an additional cost. Choosing the phases $\xi_{n}(t)$ of the evolved states in the unitary evolution operator (11) has an impact on the energy cost. Specifically $\Sigma_{H}(t)$ is minimized by setting $\dot{\xi}_{n}(t)=$ $-i\langle\dot{n}(t) \mid n(t)\rangle$ (Santos and Sarandy, 2018). In the framework of Eqs. (13)-(20) this amounts to applying $H_{C D}$ alone, $H=H_{C D}$.

Zheng et al. (2016) and Campbell and Deffner (2017) defined differential costs, instantaneous or accumulated, in terms of the $\mathrm{CD}$ term only, ignoring $H_{0}$. In particular, existence problems are circumvented by considering the state-specific counterdiabatic term $H_{C D}^{[n]}$ in Eq. (25). Note that $\left\|H_{C D}^{[n]}\right\|=\hbar \sqrt{2\langle\dot{n} \mid \dot{n}\rangle}$ exists in systems where $\|H\|$ does not. Campbell and Deffner (2017) applied "quantum speedlimit inequalities" to driving by $H=H_{0}+H_{C D}^{[n]}$ combining different norm types (trace norm and Frobenius norm). Interestingly, Demirplak and Rice already considered $\left\|H_{C D}\right\|$ and $\left\|H_{C D}^{[n]}\right\|$ as a measure of the "intensity" of the counterdiabatic terms and used them to find "minimal" $\mathrm{CD}$ terms. The state-specific $H_{C D}^{[n]}$ is thus less intense (costly) than the general purpose $H_{C D}$ (Demirplak and Rice, 2008).

Other "differential costs" have been defined using different references. In an STA protocol driven by $H(t)$ with instantaneous eigenvalues $\mathrm{E}_{n}(t)$ and an initial state which is diagonal in the $|\mathrm{n}(t)\rangle$ eigenbasis of $H(t)$, with initial probabilities $p_{n}(0)$, del Campo, Goold, and Paternostro (2014) defined a work distribution and the corresponding average work as $\langle W\rangle=\sum_{k, n}\left[\mathrm{E}_{k}(t)-\mathrm{E}_{n}(0)\right] p_{n k}^{t} p_{n}^{0}$, which is $\langle H(t)\rangle-\langle H(0)\rangle$ for such states. $p_{n k}^{t}=|\langle\mathrm{k}(t)|U(t)| \mathrm{n}(0)\rangle|^{2}$ is the probability for the system to start at $|\mathrm{n}(0)\rangle$ and be found at $|\mathrm{k}(t)\rangle$ at time $t$. Similarly, they define the "adiabatic work" $\left\langle W_{a d}(t)\right\rangle=$ $\sum\left[\mathrm{E}_{n}(t)-\mathrm{E}_{n}(0)\right] p_{n}^{0}$ and suggest as a "pragmatic" definition of cost the time average of the differential $\delta W(t)=$ $\langle W(t)\rangle-\left\langle W_{a d}(t)\right\rangle$. A lower bound $\sim t_{f}^{2}$ for STA processes with inverse engineered time-dependent frequencies was found by Cui, Chen, and Muga (2016).

In CD-driven processes, the eigenenergies of $H(t), \mathrm{E}_{n}(t)$ differ in general from the eigenenergies of $H_{0}(t), E_{n}(t)$. Similarly, eigenstates of $H(t),|\mathrm{n}(t)\rangle$, and $H_{0}(t),|n(t)\rangle$ differ in general, although in most processes $H_{C D}=0$ is imposed at $t=0$ and $t_{f}$, so that the initial and final eigenvalues and eigenvectors of $H_{0}$ and $H$ coincide. Funo et al. (2017) redefined $\left\langle W_{a d}(t)\right\rangle=\sum\left[E_{n}(t)-E_{n}(0)\right] p_{n}^{0}$ in terms of $H_{0}$ eigenvalues (this would agree with the previous definition at time $t_{f}$ ) and considered the work distributions

$$
\begin{aligned}
P[W(t)] & =\sum_{k, n} p_{n}^{0} p_{n \rightarrow k}^{t} \delta\left\{W(t)-\left[\mathrm{E}_{k}(t)-E_{n}(0)\right]\right\}, \\
P_{a d}[W(t)] & =\sum_{n} p_{n}^{0} \delta\left[W(t)-W_{a d}^{(n)}\right],
\end{aligned}
$$

where $W_{a d}^{(n)}(t)=E_{n}(t)-E_{n}(0)$. Since the initial state density operator is assumed diagonal in $\{|n(0)\rangle\}$ it will be diagonal in $\{|n(t)\rangle\}$ for all time because of the CD driving. As $\langle n(t)|H(t)| n(t)\rangle=\left\langle n(t)\left|H_{0}(t)\right| n(t)\right\rangle$ for all times, it follows that $\langle H(t)\rangle=\left\langle H_{0}(t)\right\rangle$ for all times and thus $\langle W(t)\rangle=$ $\left\langle W_{a d}(t)\right\rangle$ during the process. As for the distributions, they 
coincide at boundary times $t=0$ and $t_{f}$. Funo et al. (2017) also considered the work fluctuation with respect to the adiabatic trajectory

$$
\delta(\Delta W)^{2}=\sum_{m, n} p_{n}^{0} p_{n \rightarrow m}^{t}\left[\mathrm{E}_{m}(t)-\left.E_{n}(t)\right|^{2},\right.
$$

and found that

$$
t_{f} \geq \frac{\hbar \mathcal{L}\left[\rho(0), \rho\left(t_{f}\right)\right]}{\delta \bar{\Delta} W}
$$

where now $\mathcal{L}$ is the Bures length between initial and final (mixed) states. Funo et al. (2017) identified $\overline{\delta \Delta W}$ as the thermodynamic cost to implement the $\mathrm{CD}$ driving. The inequality (112) is tighter than the Aharonov-Anandan relation, which involves instead the fluctuation $\left\langle H^{2}(t)\right\rangle-$ $\langle H(t)\rangle^{2}$, although the latter is of broader applicability, since it is not restricted to CD driving [nevertheless Bukov, Sels, and Polkovnikov (2019) conjectured, and validated for some models, that the quantum speed limit for all protocols is bounded by the quantum speed limit for $\mathrm{CD}$ protocols]. An experimental demonstration was carried out with an Xmon qubit (Z. Zhang et al., 2018). These results were also extended to classical systems (Bravetti and Tapias, 2017). Funo, Shiraishi, and Saito (2019) studied a quantum speed limit for open quantum systems described by the Lindblad master equation. They found a "velocity term" that when the thermal relaxation is dominant compared to the unitary dynamics of the system is approximated by the energy fluctuation of the counterdiabatic Hamiltonian.

A related trade-off relation between time, entropy, and state distance was worked out by Takahashi (2017a) considering a canonical equilibrium state in density operator form $\rho(0)$, corresponding to a Boltzmann distribution with temperature $T$ as the initial state. Removing the contact with the thermal bath, this evolves unitarily by $H(t)=H_{0}(t)+H_{C D}(t)$ into $\rho(0 \rightarrow t)$. Let $\rho(t)$ be the instantaneous equilibrium state corresponding to $H(t)$, and $\rho_{0}(t)$ the equilibrium state for $H_{0}$, both at the same temperature as the initial state. Using the Kullback-Leibler divergence, or relative entropy, from $\rho_{1}$ to $\rho_{2}, D_{K L}=\operatorname{tr} \rho_{1} \ln \rho_{1}-\operatorname{tr} \rho_{1} \ln \rho_{2}$, Takahashi (2017a) found the "Pythagorean relation"

$$
\begin{aligned}
& D_{K L}(\rho(0 \rightarrow t) \| \rho(t)) \\
& \quad=D_{K L}\left(\rho(0 \rightarrow t) \| \rho_{0}(t)\right)+D_{K L}\left(\rho_{0} \| \rho(t)\right),
\end{aligned}
$$

which is interpreted as a decomposition of entropy production. A trade-off relation previously mentioned follows from setting a lower bound to it.

Abah and Lutz (2017) considered STA expansions and compressions of a harmonic trap holding a particle in the context of microscopic quantum engines and refrigerators, see Fig. 12, making use only of different time dependences for $\omega(t)$. A reference Hamiltonian $H_{0}(t)$ is complemented by some modification term $H_{\mathrm{STA}}(t)$ [found by a unitary equivalence from a CD term as explained, e.g., by S. Ibáñez et al. (2012), see Sec. II.B.2.a and Eq. (61)], so that the resulting total $H(t)$ drives an STA process. They associated the energy

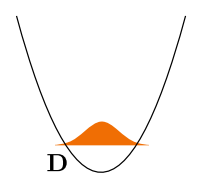

(3) Isentropic expansion Work done $W_{3}$

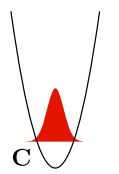

(4) Cold isochore Heat removed $Q_{4}$
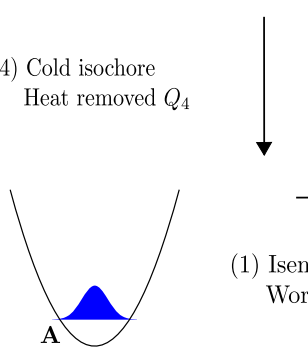

(1) Isentropic compression Work done $W_{1}$

(2) Hot isochore Heat added $Q_{2}$

FIG. 12. Quantum Otto cycle for a particle in a harmonic oscillator. The colors are intended to represent the temperature ordering as (c) red, (d) orange, (b) light blue, and (a) deep blue. Adapted from Abah and Lutz, 2018.

cost with the time-averaged $\overline{\left\langle H_{\text {STA }}\right\rangle}$. They also proposed to add these terms (one for the compression step $\overline{\left\langle H_{\mathrm{STA}}^{1}\right\rangle}$ and one for the expansion $\left.\overline{\left\langle H_{\mathrm{STA}}^{3}\right\rangle}\right)$ in the denominator of the efficiency of an Otto cycle to the heat added,

$$
\eta=\frac{\text { work output }}{\text { heat added }+\overline{\left\langle H_{\mathrm{STA}}^{1}\right\rangle}+\overline{\left\langle H_{\mathrm{STA}}^{3}\right\rangle}},
$$

as an extra energy input. Thus this approach ignores the possible role of $H_{0}(t)$ in actual energy costs and leads to some paradoxical results. In particular, choosing $H(t)=$ $H_{0}(t)+H_{\text {STA }}(t)$ as the new reference $H_{0}^{\prime}(t)=H(t)$, with frequency $\omega^{\prime}(t)$ given by Eq. (61), no extra term is needed since $H_{0}^{\prime}(t)$ is already a shortcut. The efficiency, being of a differential nature, would then have different values for the same process depending on how the Hamiltonian is partitioned (in one, $H_{0}^{\prime}=H$, or two pieces, $H_{0}+H_{\mathrm{STA}}$ ).

If the unitary transformation on $H_{C D}$ is not performed (Abah and Lutz, 2018; Abah and Paternostro, 2018), the same type of modified efficiency is proposed with the time averaged $\overline{\left\langle H_{C D}\right\rangle}$ playing the role of $\overline{\left\langle H_{\mathrm{STA}}\right\rangle}$. However, $\left\langle H_{C D}(t)\right\rangle$ is zero by construction at all times for states diagonal in the eigenbasis of $H_{0}(t)$; see Eq. (14). Accordingly, all processes would have the same zero cost independently of the $H_{0}(t)$ chosen.

Several papers analyzed the effect of a control system (CS) (also termed driving or auxiliary system) coupled to the primary system (PS) of interest to set the STA driving and its influence on the energy cost. Calzetta (2018) pointed out that the time-dependent driving Hamiltonians in STA processes are typically semiclassical and thus approximate. For a simplified model with a particle in a harmonic oscillator whose frequency depends on a coordinate of a driving system and is subjected to quantum fluctuations, Calzetta estimated the excitation when the STA process is implemented on average. It grows inversely with process time, but also vanishes as the mass of the driving system increases. In a different vein, Horowitz and Jacobs (2015), for a model in which the mesoscopic PS system of interest is coupled to 


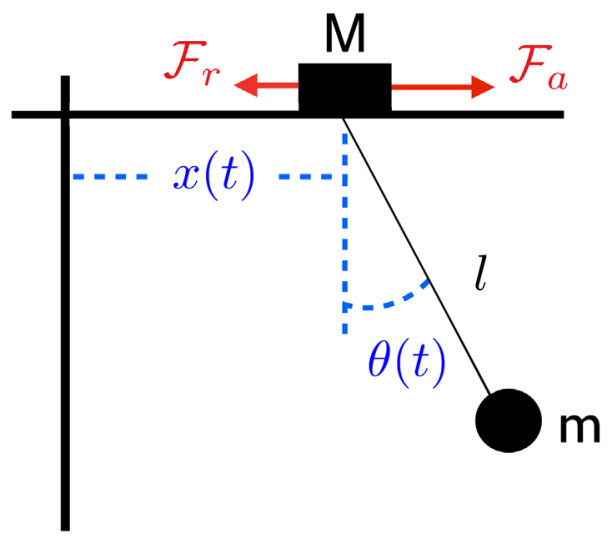

FIG. 13. Overhead crane composed of a load of mass $m$ (the primary system PS) and a trolley of mass $M$ (the control system CS) connected through a rope of constant length $l$. The solid red arrows represent the active force $\mathcal{F}_{a}$ and the friction force $\mathcal{F}_{r}$ acting on a rightward-moving trolley. Adapted from Torrontegui et al., 2017.

dissipative Markovian noise processes and to an auxiliary control system connected to a thermal bath, argued that the minimal work to drive the system through a specified path of states is due to the need for the controller to compensate for the dissipation that tries to take the system away from the path. The exact formula depends on the Hamiltonian and state of the primary system for weak coupling but on the full Hamiltonian and state (including CS + PS) for strong coupling.

Torrontegui et al. (2017), Tobalina, Alonso, and Muga (2018), and Tobalina, Lizuain, and Muga (2019) also stressed the importance of the control system to find out true energy consumptions. The control system here is the equipment necessary to set the values of the control parameters of the primary system. This equipment is usually macroscopic and behaves classically. Even without direct dissipation of the primary system, implementing the driving will require energy consumption due to an external force, invested to change the energy of the global system (PS + CS) and to combat friction of the control system; see Sec. VII.B.1 and Fig. 13. Since STA processes are by definition fast, the arguments to justify minimal energy consumptions neglecting friction in the limit of slow motion (Landauer, 1961) are not of much value. ${ }^{12}$ In a scenario with macroscopic control system and microscopic primary system, driving the control system along a predetermined path $x(t)$ is likely to be the dominant source of consumption, with the energy and backaction of the primary system being negligible in comparison, e.g., because of a large CS mass $M$ compared to the PS mass $m$. These and other effects are indeed shown explicitly with models for transporting a classical load (Torrontegui et al., 2017) or an ion in a

\footnotetext{
${ }^{12}$ For an analysis of the work required by shortcuts to erase a bit in finite time beyond Landauer's bound, see Boyd et al. (2018). The model used is a Brownian particle in a double well potential. Instead of a trade-off between information and energy, as in Landauer's work, more complex trade-offs are found that depend on information and its robustness, energy, statistical bit-bias difference, size of the memory states, and speed.
}

multisegmented Paul trap (Tobalina, Alonso, and Muga, 2018; Tobalina, Lizuain, and Muga, 2019). A further relevant observation in these works is that in practice both positive and negative powers of the external force typically imply consumption, i.e., the energy given away by the system in "braking" time segments with a negative power is not stored and recovered at will in positive-power segments, although a phenomenological parameter is introduced to account for the possibility to perform, at least partially, energy-efficient regenerative braking. The models also show the importance of the control system to find the experimental gauge to determine the PS energy and corresponding power. For example, in a simple harmonic transport of a particle with Hamiltonian $p^{2} /(2 m)+m \omega^{2}\left[x-x_{0}(t)\right]^{2} / 2+g(t)$, driven by the control function $x_{0}(t)$, the gauge function $g(t)$ does not affect the dynamics and so it is frequently ignored, but it may strongly affect the PS energy and corresponding power (Campisi, Hänggi, and Talkner, 2011; Tobalina, Lizuain, and Muga, 2019). A similar effect is found in expansions and compressions of an ion in a Paul trap, where the gauge term implies the opposite behavior to what could be naively expected, namely, an increase of energy during the expansion, and a decrease during the compression. It remains to be seen if smart engineering and design can equate the power of the external force to the PS power (this implies unrealistic assumptions in the worked out models, such as $M=0$ and no friction), or at least make them proportional, as approximately realized in some model examples (Tobalina, Alonso, and Muga, 2018; Tobalina, Lizuain, and Muga, 2019). In any case further analysis of energy consumption, both fundamental and for STA processes in different systems (Impens and GuéryOdelin, 2019), is needed.

\section{B. Engines and refrigerators}

A fundamental problem to design heat engine cycles is that maximal thermal efficiency (output work divided by the heat input from the hot bath) is achieved with slow processes that minimize losses but also imply negligible power output (output work divided by cycle time). Shortcuts appear at first sight to solve this problem as the adiabatic evolution can be mimicked in short times. STA-driven engines or refrigerators have been considered mostly for harmonic oscillators performing Otto cycles, see Fig. 12, with two isochoric (constant frequency) branches for contact with the hot and cold baths and thermally isolated compression or expansion branches where STA driving, as developed, e.g., in Salamon et al. (2009) and Chen, Lizuain et al. (2010), is applied. ${ }^{13}$ For a recent review of the quantum Otto cycle for engines or refrigerators, see Kosloff and Rezek (2017).

Before the term shortcuts to adiabaticity existed, Ronnie Kosloff's group had already worked out "frictionless" bangbang protocols for the isochoric strokes in which the adiabatic

\footnotetext{
${ }^{13} \mathrm{~A}$ version of the Otto cycle using single- and two-spin- $1 / 2$ systems was proposed by Çakmak and Müstecaplıoğlu (2018). An STA-enhanced Otto refrigerator based on a superconducting qubit with continuous coupling to two resonant circuits was analyzed by Funo et al. (2019).
} 
state was recovered in the final time; see, e.g., Rezek et al. (2009) and Salamon et al. (2009). After 2010, two early studies on shortcuts applied to cycles were Torrontegui and Kosloff (2013), which examined the performance of a refrigerator subject to noise, and Deng et al. (2013), which addressed classical and quantum systems to boost work characteristics and overall heat engine performance.

Many other works followed and studied potential advantages or optimization of STA-driven processes ( $\mathrm{Tu}, 2014$; Xiao and Gong, 2014; del Campo, Goold, and Paternostro, 2014; Abah and Lutz, 2016, 2017). Some have tried to enhance the power output by considering not just STA but also many-body systems (Beau, Jaramillo, and del Campo, 2016; Chotorlishvili et al., 2016; Jaramillo, Beau, and del Campo, 2016; Deng, Chenu et al., 2018; Deng, Diao et al., 2018), and nonlinear BECs (J. Li et al., 2018) driven by STA found with variational methods (Li, Sun, and Chen, 2016). As well, Babajanova, Matrasulov, and Nakamura (2018) found the equations of state for FF dynamics in expanding cavities that contain an ideal Fermi gas, and Villazon, Polkovnikov, and Chandran (2019) proposed STA methods (unitarily transformed counterdiabatic driving) to manipulate both trap frequency and the coupling to the environment to realize fast approximate Otto engines operating near Carnot efficiency.

The role and design of STA methods in quantum engines and refrigerators is very much an open field where rather fundamental questions are still under scrutiny and debate. (Further aspects are discussed in Secs. IV.C and V.) For example, most studies systematically focus on the primary system only so that efficiencies and power computed with usual PS-based formulas are idealized limits that ignore CS effects discussed in the previous section. Some tentative proposals exist to modify the denominator (energy input) in the efficiency, such as taking into account time-averaged interactions of the terms added to the reference Hamiltonian to perform the STA (Abah and Lutz, 2017), energy dissipated by noise in the controls (Kosloff and Rezek, 2017), or energy consumptions to set the CS parameters (Tobalina, Lizuain, and Muga, 2019). Tobalina, Lizuain, and Muga (2019) pointed out that STA Otto engines based on a trapped ion are not ordinary engines. In the expansion stroke, the "piston," whose role is played by the harmonic trap, is not pushed by a hot ion, which exerts a negligible effect on the trap, but rather it is externally driven, in a fast manner specified by the STA protocol, by a controlled circuit that consumes and dissipates energy to do so, in fact, so much energy that the microscopic (PS) work output is far from compensating it. This may well happen for a broad domain of macroscopically controlled quantum systems. A way out might be that the quality of the microscopic work is worth the energy expense anyway, e.g., because of its effect on relevant degrees of freedom, but there is much to do to substantiate this hope in practice.

\section{Third law}

The third law of thermodynamics was formulated by Nernst as the impossibility to reduce any system to absolute zero in a finite number of operations; see Kosloff (2013) for a review. In the context of a quantum Otto heat pump it may be viewed as the vanishing of the cooling rate when the temperature of the cold bath approaches zero (Rezek et al., 2009) and be quantified by the scaling law that relates the cooling rate and cold-bath temperature. The fundamental bottleneck is the time needed for the expansion branch. For a harmonic oscillator with a time-dependent frequency, the temperature of the equilibrium states connected adiabatically (i.e., slowly) or by STA is proportional to the frequency $\hbar \omega \propto k T$ to keep the average occupation number constant. Relevant questions are how fast we can lower $\omega$ and also finding scalings between the wanted times and the resources needed. The answers are not unique and depend on the method and constraints imposed. For expansions limited to a designed protocol for $\omega(t)$ minimal times exist if $\omega(t)$ is real and bounded. Simple bang-bang solutions for real $\omega$ give $t_{f} \sim \omega_{c}^{-1 / 2}$ ( $\omega_{c}$ being the extreme, target value of $\omega$ in the expansion) (Rezek et al., 2009; Salamon et al., 2009; Stefanatos, 2017b). If $\omega(t)$ is not restricted and allowed to be imaginary, the expansion times can be formally arbitrarily short for any $\omega_{c}$ (Chen, Lizuain et al., 2010). However, it is unrealistic to assume that arbitrarily fast processes are viable, as variously argued in Sec. IV.A. In particular, if the time average of the energy is supposed to be bounded, since the trap depth cannot be arbitrarily high, the same type of scaling arises (Chen and Muga, 2010). In fact more sophisticated bang-bang solutions, allowing for imaginary frequencies (Hoffmann et al., 2011), or an arbitrary number of switches (Stefanatos, 2017a, 2017b) lead to faster processes.

\section{OPEN QUANTUM SYSTEMS}

In closed quantum systems, a slowly changing Hamiltonian can give rise to adiabatic dynamics as already discussed. By contrast, in open quantum systems, a slowly changing system Hamiltonian, or equivalently a long evolution time, does not necessarily guarantee adiabatic dynamics. In addition, the cumulative effect of dissipation increases with time. Shortcuts to adiabaticity in this context should therefore be defined with care. Interestingly, the control design of open quantum systems paves the way for the use of shortcuts in the emerging field of thermodynamics of quantum systems.

\section{A. Concept of adiabaticity for open systems}

A direct consequence of the coupling of the system with the environment is the need to redefine adiabaticity, as new elements and time scales appear compared to closed systems. Sarandy and Lidar (2005) generalized the adiabatic approximation to open systems for convolutionless master equations, i.e., master equations of the form $\dot{\rho}(t)=\mathcal{L}(t) \rho(t)$. Unlike closed systems, for which the Hamiltonian can always be diagonalized, the Lindblad superoperator $\mathcal{L}(t)$ of an open system is not necessarily diagonalizable and in general can be written only in Jordan normal form. Adiabaticity for open systems is subsequently defined as the regime for which the evolution of the state of a system takes place without mixing the various Jordan blocks. Alternatively, the adiabatic approximation in open systems can be formulated through an effective Hamiltonian approach (Yi et al., 2007).

Further work about adiabaticity for open systems also includes the derivation of Markovian master equations suited 
(a)
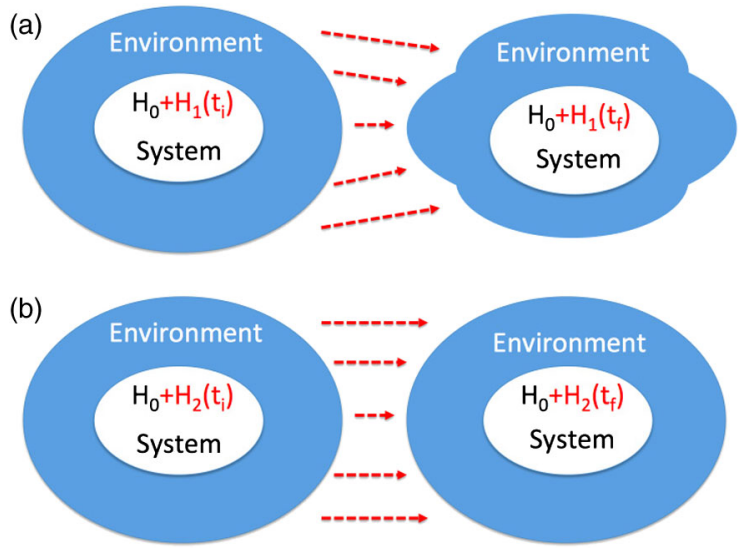

FIG. 14. Two kinds of control STA protocols of an open quantum system: (a) those involving a reservoir engineering in addition to Hamiltonian engineering, and (b) those relying only on Hamiltonian engineering.

for studying the time evolution of a system evolving slowly while coupled weakly to a thermal bath (Thunström, Åberg, and Sjöqvist, 2005; Pekola et al., 2010; Albash et al., 2012, 2015; Venuti et al., 2016; Kiely, Muga, and Ruschhaupt, 2017), and the derivation of a link between the notion of adiabaticity for open system and the theory of noiseless subsystems (Oreshkov and Calsamiglia, 2010).

Hereafter, we address different strategies to implement STA protocols in the presence of a coupling with the environment. We first discuss techniques requiring a reservoir engineering, then focus on methods set up to mitigate the effect of a (nonmanipulated) environment; see Fig. 14. We conclude on a more specific class of open systems that can be described by non-Hermitian Hamiltonians.

\section{B. Engineering the environment}

Building on the work by Sarandy and Lidar (2005), the concept of a CD driving was generalized to open systems by working out the additional term to be superimposed to the Hamiltonian to guarantee an independent evolution of the different Jordan blocks of the Lindblad operator (Vacanti et al., 2014). While this additional driving is unitary in some cases, in general it does not even provide a completely positive map.

A natural framework to extend the shortcuts to open systems is the explicit use of DFSs (S. L. Wu et al., 2017). Consider that the coupling to the environment is accounted for by a Lindblad form

$$
\mathcal{L}(t)=-\frac{i}{\hbar}[H, \rho]+\sum_{k} X_{k} \rho X_{k}^{\dagger}-\frac{1}{2}\left\{X_{k}^{\dagger} X_{k}, \rho\right\},
$$

where the Lindblad operators may be time dependent. Similar to closed systems, the dynamical invariants of an open system provide us with both an intuitive physical framework and a set of tools to engineer quantum states (Ma, Cao, and Wu, 2017). In this perspective, it is useful to establish the modified equation for the invariant, which reads in this context

$$
\partial_{t} I+\frac{i}{\hbar}[H, I]+\sum_{k} X_{k}^{\dagger} I X_{k}-\frac{1}{2}\left\{X_{k}^{\dagger} X_{k}, I\right\}=0
$$

In contrast to closed systems, although $\langle I\rangle$ is still constant in time, its eigenvalues $\lambda_{n}$ are no longer necessarily time independent. More precisely, all $\lambda_{n}$ are time independent if $I$ and $X_{k}$ have a common basis of eigenvectors for all $k$.

However, it is possible to design a type of dynamical invariants for open quantum systems, in which a part of the eigenvalues is constant in time. By construction, the corresponding eigenstates are in the time-dependent DFSs. The interest of such subspace is that a quantum state evolves unitarily in it. By definition, the vectors of the DFS $\left\{\left|\Phi_{1}\right\rangle,\left|\Phi_{2}\right\rangle, \ldots,\left|\Phi_{D}\right\rangle\right\}$ are degenerate eigenstates of the Lindblad operators that obey the relation $X_{k}(t)\left|\Phi_{j}(t)\right\rangle=$ $c_{k}(t)\left|\Phi_{j}(t)\right\rangle$ (Wu, Zhang, and Yi, 2015). A counterdiabatic driving Hamiltonian in the time-dependent DFS can be shown to be of the form (S. L. Wu et al., 2017)

$$
\tilde{H}=H(t)+\frac{i}{2} \sum_{k}\left(c_{k}^{*} X_{k}-c_{k} X_{k}^{\dagger}\right) .
$$

The driving based on invariants in the DFS can be equivalently worked out (Ma, Cao, and $\mathrm{Wu}, 2017)$. An important condition that needs to be fulfilled to control in time such quantum systems is the dynamical stability of the time-dependent DFS (S. L. Wu et al., 2017). The application to few-spin systems was explicitly worked out by Ma, Cao, and Wu (2017) and S. L. Wu et al. (2017).

We note that another approach to set up the DFS consists of defining the invariant as a superoperator $\mathcal{I}$ that fulfills the relation (Sarandy, Duzzioni, and Moussa, 2007)

$$
\frac{\partial \mathcal{I}}{\partial t}=[\mathcal{L}, \mathcal{I}]
$$

$\mathcal{I}$ is in general non-Hermitian. Decoherence-free evolution can further be constructed when the commutator of the Lindbladian and the superoperator $[\mathcal{L}, \mathcal{I}]$ is independent of the noise parameters (Sarandy, Duzzioni, and Moussa, 2007).

Reservoir engineering to shortcut the thermalization process was also discussed in the context of nonadiabatic Markov equations (Dann, Levy, and Kosloff, 2018; Dann, Tobalina, and Kosloff, 2018). Such an approach, well suited for fast driving within the Markovian approximation, properly accounts for the coupling between population and coherence and explains the emergence of coherence associated with dissipation. Systems coupled to a non-Markovian bath have also been investigated. Villazon, Polkovnikov, and Chandran (2019) detailed the driving of such system using a protocol that controls in time both the system parameters and the coupling strength to the bath. This protocol was further exploited in an Otto-like engine operating at high power.

\section{Mitigating the effect of environment}

Quite often the coupling with the environment and the environment itself cannot be designed. For instance, the environment may induce noise in some parameters 
(Ruschhaupt et al., 2012; Kiely, Muga, and Ruschhaupt, 2017). In such a scenario STA techniques may be designed to mitigate its effects. Sun et al. (2016) investigated different variants of $\mathrm{CD}$ driving for a finite-time Landau-Zener process in the presence of a bath. Other strategies exploit the freedom on the phases $\xi_{n}(t)$ for the evolution operator $U(t)=$ $\sum_{n} e^{i \xi_{n}(t)}|n(t)\rangle\langle n(0)|$ to minimize the effect of the coupling with the environment (Santos and Sarandy, 2018) in CD driving. In the same spirit, STA based on invariants can be readily adapted along the lines set in Ruschhaupt et al. (2012); see Sec. II.H. Levy, Torrontegui, and Kosloff (2017) minimized the effect of noise by reducing at best the commutators between invariants and the Lindblad noise operators $X_{k}$, specifically by minimizing the quantities $\mathcal{A}_{k} \sim$ $\int_{0}^{t_{f}} d s\left\|\left[X_{k}(s), I(s)\right]\right\|$ over the process duration. Designing an invariant $I$ that commutes with the noise operators $X_{k}$ ensures that populations do not decay in the invariant eigenbasis and that decay of coherences is reduced. It is not always possible to minimize all $\mathcal{A}_{k}$ simultaneously, so a weighted average is minimized instead. Similarly, starting from an inverse engineering protocol, the parameters can be shaped in time to enforce the robustness against stochastic fluctuations in the Hamiltonian for a wide class of noise types (Jing et al., 2013).

\section{Non-Hermitian Hamiltonians}

There is a class of quantum systems for which the environment can be modeled using non-Hermitian Hamiltonians. Such Hamiltonians usually describe subsystems of a larger system (Muga et al., 2004). Complex energies imply that "adiabaticity theorems" apply only to weak non-Hermiticity regimes or to the least dissipative state (Nenciu and Rasche, 1992). Moreover, since right and left eigenvectors are normalized in a biorthogonal sense, the normalization factors are ambiguous and care must be exercised to extend the concept of "population" to define an adiabaticity criterion (Ibáñez and Muga, 2014).

Concerning the standard STA techniques, the counterdiabatic driving was generalized to weak non-Hermitian Hamiltonians (Ibáñez, Martínez-Garaot et al., 2011; Ibáñez et al., 2012; Chen, Xia et al., 2016; J. Song et al., 2016; G.-Q. Li et al., 2017; H. Li et al., 2017; Chen, Wu et al., 2018). Ibáñez, Martínez-Garaot et al. (2011), in particular, applied the formalism to control a decaying two-level system. Later Torosov, Della Valle, and Longhi (2013) demonstrated that auxiliary gain and loss imaginary terms added in the diagonal of the Hamiltonian of Hermitian two-level models, which are feasible in waveguide optics, can be chosen to cancel nonadiabatic transitions and perform fast population transfers. The results were also generalized to three-level systems (Torosov, Della Valle, and Longhi, 2014; Q.-C. Wu et al., 2016; G.-Q. Li et al., 2017) and applied to a two-level system coupled to a dissipative spin chain (Diffo et al., 2017). In Impens and Guéry-Odelin (2019), the extra driving field one has to superimpose to the original one to compensate for the distortion in the spin-1/2 direction on the Bloch sphere due to a dissipative non-Hermitian term, is explicitly worked out.

As for Lewis-Riesenfeld invariants for non-Hermitian Hamiltonians, they can be generalized in two different forms
(Simón, Buendía, and Muga, 2018) corresponding to (Gao, $\mathrm{Xu}$, and Qian, 1992; Khantoul, Bounames, and Maamache, 2017; Maamache et al., 2017)

$$
\begin{aligned}
\frac{\partial I}{\partial t}+\frac{i}{\hbar}[H, I] & =0, \\
\frac{d}{d t}\langle\hat{\psi}(t)|I(t)| \psi(t)\rangle & =0,
\end{aligned}
$$

where $\hat{\psi}(t)$ evolves with $H^{\dagger}(t)$ or to

$$
\begin{aligned}
\frac{\partial I^{\prime}}{\partial t}+\frac{i}{\hbar}\left[H^{\dagger}(t) I^{\prime}(t)-I^{\prime}(t) H(t)\right] & =0, \\
\frac{d}{d t}\left\langle\psi(t)\left|I^{\prime}(t)\right| \psi(t)\right\rangle & =0 .
\end{aligned}
$$

Combined with inverse engineering, the first option was considered, e.g., by Ibáñez, Martínez-Garaot et al. (2011) and Luo et al. (2015), and the potential of the second option is yet to be explored.

\section{OPTICAL DEVICES}

There are some proposals to make use of time-dependent parameters to control light waves propagating in linear media via STA approaches (Lakehal, Maamache, and Choi, 2016), but the bulk of STA applications in optics substitutes time by a spatial coordinate. Thus shortcuts to adiabaticity in optics can lead to more compact waveguide devices, which favors device integration. After numerous theoretical works, the stage where actual STA-enhanced devices are constructed has just been reached.

STA applications in optics began exploiting the analogies (Longhi, 2009) between the electromagnetic wave propagation inside a waveguide in the paraxial approximation and the propagation of a quantum wave function inside a well. Consider a stationary scalar field $E(\mathbf{r})$ that satisfies the Helmholtz equation, a time-independent form of the wave equation,

$$
\nabla^{2} E(\mathbf{r})+n^{2}(\mathbf{r}) k^{2} E(\mathbf{r})=0
$$

where $n(\mathbf{r})$ is the position-dependent refractive index inside the waveguide and $k=2 \pi / \lambda$, with $\lambda$ the light wavelength in vacuum. Two common approximations are the small angle or paraxial approximation, which assumes the form

$$
E(\mathbf{r})=\mathcal{E}(\mathbf{r}) e^{i k_{z} z}
$$

and a slowly varying envelope approximation (SVEA) in the scale of $2 \pi / k_{z}$ to neglect $\partial^{2} \mathcal{E} / \partial z^{2} . k_{z}=k n_{0}$ plays the role of an "optical mass" and $n_{0}$ is the outer (bulk) refractive index. These approximations lead to a simplified form of Eq. (120) with the same form as the time-dependent Schrödinger equation, and the longitudinal space $z$ coordinate playing the role of time,

$$
i \frac{\partial \mathcal{E}}{\partial z}=\left[-\frac{1}{2 k n_{0}} \nabla_{\perp}^{2}+V(\mathbf{r})\right] \mathcal{E},
$$




$$
V(\mathbf{r})=\frac{k}{2 n_{0}}\left[n_{0}^{2}-n^{2}(\mathbf{r})\right]
$$

Coupled-mode theory.-Beam dynamics in coupled waveguides is usually addressed with "coupled-mode theory." Among the different formulations we focus here on the most used one in STA applications. Assume $N$-coupled waveguides, where the refractive index and geometry of the waveguides are allowed to vary along the propagation direction $z$. Under the scalar and paraxial approximations and the assumption of weak coupling, the variations of the guided-mode amplitudes of individual waveguides $\mathbf{A}=$ $\left[a_{1}, a_{2}, \ldots, a_{N}\right]^{T}$ with propagation distance are described by the coupled-mode equations

$$
i \frac{d \mathbf{A}}{d z}=H(z) \mathbf{A} .
$$

Once again, replacing the spatial variation $z$ with the temporal variation $t$, Eq. (124) is equivalent to the time-dependent Schrödinger equation $(\hbar \equiv 1)$ describing the dynamics of an $\mathrm{N}$-state system, with $H(z)$ playing the role of the Hamiltonian.

Lin et al. (2012) discovered the use of STA protocols to stabilize and reduce the size of coupled waveguide systems, applying the $\mathrm{CD}$ driving approach to the mode conversion in a multimode waveguide with three coupled modes. The results using coupled-mode theory were checked using the so-called wide-angle beam propagation numerical method, which solves the Helmholtz equation with SVEA, but no paraxial approximation.

Numerous works followed this line. Many of the methods presented in Sec. II were used to improve mode converters (Tseng and Chen, 2012; Tseng, 2013; Yeih, Cao, and Tseng, 2014) and also to design and optimize other devices such as mode-division multiplexing systems (Martínez-Garaot, Tseng, and Muga, 2014), see Fig. 15, directional couplers (Tseng, 2014; Tseng et al., 2014), or polarization rotators (X. Chen et al., 2014).

Stefanatos (2014) studied how to implement an STA in an optical semi-infinite system. A semi-infinite photonic lattice was designed to drive the input light to a controlled location at the output using the invariant-based inverse engineering approach, interpreting the photonic lattice as a quantum harmonic oscillator with time-dependent mass. One year later, Pan and Tseng (2015) applied the theory in Tseng et al. (2014) to design silicon-based platforms with high refractive index contrast, Paul and Sarma (2015) used the CD approach to improve the design of a directional coupler, and Ho and Tseng (2015) optimized the adiabaticity of coupled-waveguide devices using invariant-based inverse engineering.

Della Valle, Perozziello, and Longhi (2016) developed an STA for ultracompact waveguide junctions inspired by the streamlined version of the fast-forward approach (Torrontegui, Martínez-Garaot et al., 2012). In this way they went beyond the coupled-mode equation formalism by extending the optical STA to full-wave problems for the Helmholtz equation, i.e., to an infinite-dimensional system.

Chen, Wen, and Tseng (2016) used the invariants to design stable directional couplers against errors in input wavelength

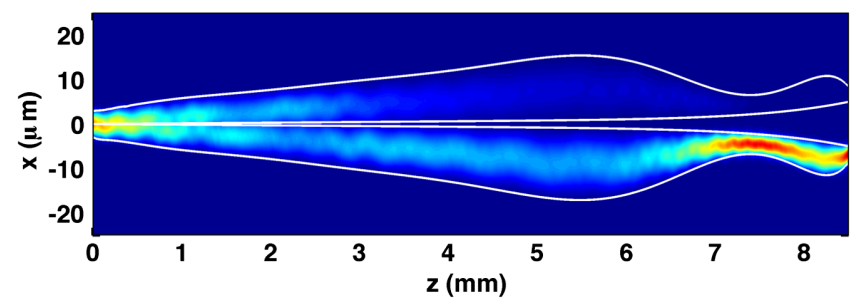

(a)

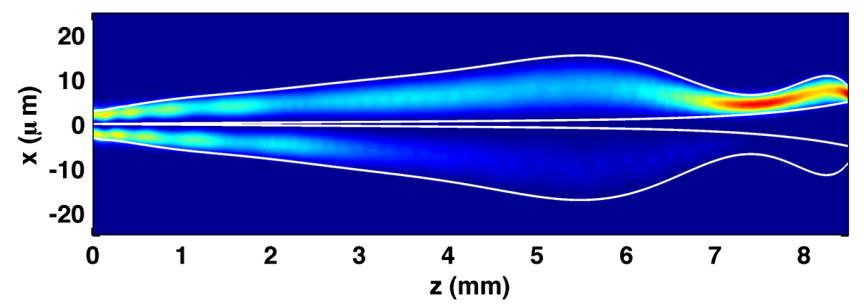

(b)

FIG. 15. Mode-sorting operation of the invariant-based Y junction. Input (a) fundamental mode and (b) second mode. From Martínez-Garaot, Tseng, and Muga, 2014.

and coupling coefficient simultaneously. Later MartínezGaraot, Muga, and Tseng (2017) adapted the FAQUAD approach to the optical devices to design mode-sorting asymmetric $\mathrm{Y}$ junctions. This approach is quite useful in this context because the information it requires is accessible, and the simplicity of changing only one control parameter is ideal for device fabrication. Besides, Chung, Lee, and Tseng (2017) designed a short and broadband silicon asymmetric Y-junction two-mode (de)multiplexer using the theory by MartínezGaraot, Muga, and Tseng (2017) and Wu, Ji, and Zhang (2017c) developed the generation of 3D entanglement using dressed states, and Huang, Ang, and Kyoseva (2017) applied the counterdiabatic approach to design shorter and robust two- and three-waveguide couplers. Finally, Chen, Wen et al. (2018) used the Lie-transform theory by Martínez-Garaot et al. (2014) to design compact beam splitters.

In some recent works dealing with silicon waveguides with high index contrast, the scalar and paraxial approach is not accurate, so commercial software is used to solve the dynamics. Specifically, in Martínez-Garaot, Muga, and Tseng (2017) the shortcut was done using the scalar Helmholtz equation without the paraxial approximation and in Chung, Lee, and Tseng (2017) and Chung and Tseng (2018) the FAQUAD approach was applied without approximations, since it can incorporate the vectorial fields into the calculations without resorting to the coupled-mode equations. Moreover, Della Valle (2018) used shortcuts beyond the paraxial limit to achieve efficient rejection of higher order modes in a broad wavelength range for any two-dimensional multimode optical waveguide. Non-Hermitian systems with gain and loss were also considered (Torosov, Della Valle, and Longhi, 2013, 2014; Longhi, 2017).

The first experimental implementation of STA in optical devices was worked out by Guo and Chu (2017) demonstrating broadband silicon mode (de)multiplexers with optimized tapers using the method by Ho and Tseng (2015), and recently, the group of S.-Y. Tseng (Hung et al., 2019) produced devices using the FAQUAD approach. 


\section{EXTENSION TO CLASSICAL AND STATISTICAL PHYSICS}

In this section, we reviewed the different STA methods which have been extended to classical mechanics and statistical physics, along with the presentation of a few proof-of-principle experiments that have been carried out to demonstrate their effectiveness.

\section{A. Counterdiabatic methods in classical mechanics}

As already discussed in Sec. II.B, the counterdiabatic term that we add to a time-dependent Hamiltonian in quantum mechanics inhibits any excitation in the system. A natural question is how we can transpose these ideas to a classical system, and to what extent they are related to their quantum counterpart.

We first address this question in the context of one-body classical mechanics. The essence of the method can be readily explained with a 1D integrable system described by a Hamiltonian $H_{0}(p, x, \lambda)$, where $\lambda$ can be a multicomponent vector (for simplicity, we use a single component). Using a canonical transformation, there exist angle-action coordinates $(\theta, I)$ such that $H_{0}(p, x, \lambda)=\tilde{H}_{0}(I, \lambda)$, i.e., the Hamiltonian expressed in terms of the action-angle variables is independent of the angle $\theta$ as a result of its integrability. Assuming that the parameter is now time dependent, the new Hamiltonian resulting from the canonical transformation in action-angle variables takes the form (Goldstein, Poole, and Safko, 2002)

$$
H(I, \theta, t)=\tilde{H}_{0}(I, \lambda)+\partial_{t} F=\tilde{H}_{0}(I, \lambda)+\dot{\lambda} \partial_{\lambda} F
$$

where $F$ is a time-dependent generator whose "type" depends on its variables (Goldstein, Poole, and Safko, 2002; Deng et al., 2013; Deffner, Jarzynski, and del Campo, 2014; Kolodrubetz et al., 2017). For instance, $F=F_{1}(x, \theta, t)$ for type I. From Eq. (125), it is clear that the counterdiabatic Hamiltonian that one may superimpose to keep I constant with fast changes in $\lambda$ is $H_{C D}=-\partial_{t} F$ (Deng et al., 2013). In the adiabatic limit $(\dot{\lambda} \rightarrow 0)$, the Hamiltonian $H$ boils down to $H_{0}$. The counterdiabatic Hamiltonian $H_{C D}$ added to the original Hamiltonian ensures that the volume of the phase space enclosed by a given energy shell remains constant despite the variation in time of the parameters (Jarzynski, 2013).

We now present some illustrative examples. Consider a 1D harmonic oscillator whose angular frequency $\omega(t)$ is time dependent:

$$
H_{0}(p, x, t)=\frac{p^{2}}{2 m}+\frac{1}{2} m \omega^{2}(t) x^{2} .
$$

For such a Hamiltonian, the adiabatic criterion is

$$
\frac{d \omega}{d t} \ll \omega^{2}
$$

Using the type-I generating function, the action-angle canonical transformation yields $p=\partial_{x} F_{1}, I=-\partial_{\theta} F_{1}$, and $H(I, \theta, t)=\tilde{H}_{0}(I, \lambda)+\dot{\omega} \partial_{\omega} F_{1}$ (Goldstein, Poole, and Safko,
2002). To get an expression of $H_{0}$ independent of $\theta$, a natural choice is to search $x$ and $p$ in the form $p=f(I) \cos (\theta)$ and $x=[f(I) \sin \theta] / m \omega$ where the function $f(I)$ needs to be determined. We readily find $f(I)=(2 \operatorname{Im} \omega)^{1 / 2}, F_{1}(x, \theta, t)=$ $\left(m \omega x^{2} \cot \theta\right) / 2=p x / 2$, and $H_{0}(I, t)=I \omega$ and deduce $H_{C D}(p, x, t)=-\dot{\omega} F_{1} / \omega=-\dot{\omega} p x /(2 \omega)$. This result coincides perfectly with the classical limit of the quantum results (see Sec. II.C.2) (Muga et al., 2010). The calculation performed here on a harmonic potential can be readily generalized to even-power-law potentials and to a particle in a one-dimensional box (Jarzynski, 2013).

We now consider the following example of the transport of a particle by moving a harmonic trap ${ }^{14}$ :

$$
H_{0}(p, x, t)=\frac{p^{2}}{2 m}+\frac{1}{2} m \omega^{2}\left[x-x_{0}(t)\right]^{2} .
$$

We can again work out the canonical transformation to recast the problem in terms of action-angle variables by replacing $x$ by $x-x_{0}(t)$ in the previous calculation. The transformed Hamiltonian now reads $H=\tilde{H}_{0}(I, \lambda)+\dot{x}_{0} \partial_{x_{0}} F_{1}=$ $\tilde{H}_{0}(I, \lambda)-p \dot{x}_{0}$. The counterdiabatic Hamiltonian is therefore $H_{C D}(p, x, t)=p \dot{x}_{0}$ (Sels and Polkovnikov, 2017), as in the quantum result (Torrontegui et al., 2011). The quantum unitary transformation (Ibáñez et al., 2012) to find an alternative local interaction corresponds classically to an additional canonical or gauge transformation. This procedure is referred to as the local counterdiabatic driving (Ibáñez et al., 2012; Deffner, Jarzynski, and del Campo, 2014; Sels and Polkovnikov, 2017).

For a 1D Hamiltonian, a gauge transformation involves only a scalar potential $\chi(x, t)$. The momentum $p$ is transformed as $\tilde{p}=p+\partial_{x} \chi$ for the Hamiltonian $\tilde{H}=H+$ $\partial_{t} \chi$. For our first example, the gauge function $\chi(x, t)=$ $\dot{\omega} m x^{2} /(4 \omega)$ yields a gauged transformed Hamiltonian $\tilde{H}$ which is that of a harmonic oscillator of effective timedependent angular frequency $\omega_{\text {eff }}=\left[\omega^{2}+\ddot{\omega} /(2 \omega)-3 \dot{\omega}^{2} /\right.$ $\left.\left(4 \omega^{2}\right)\right]^{1 / 2}$, which coincides with its quantum counterpart; see Eq. (61) (Ibáñez et al., 2012). For the second example, the gauge function $\chi(x, t)=-m \dot{x}_{0} x$ yields $\tilde{H}(p, x, t)=$ $p^{2} /(2 m)+(1 / 2) m \omega^{2} x^{2}-m \ddot{x}_{0} x$. The extra force $F=m \ddot{x}_{0}$ superimposed to the static Hamiltonian plays the role of an effective gravitational field whose amplitude is proportional to the acceleration $\ddot{x}_{0}$ and compensates for the inertial force in the frame attached to the potential.

Beyond the two specific examples previously detailed, the formalism can be generalized to scale-invariant systems, i.e., Hamiltonians of the form (Deffner, Jarzynski, and del Campo, 2014)

$$
H_{0}(p, x, t)=\frac{p^{2}}{2 m}+\frac{1}{\gamma^{2}(t)} U\left(\frac{x-x_{0}(t)}{\gamma(t)}\right),
$$

where $\gamma(t)$ and $x_{0}(t)$ are real functions depending on time. Shortcuts are not restricted to scale-invariant systems as

\footnotetext{
${ }^{14}$ The same formalism still holds for an arbitrary transport potential.
} 
explicitly shown by Patra and Jarzynski (2017a) on a simple example. The counterdiabatic Hamiltonian is also not unique. For instance, we have worked out its form linear in $p$ for transport, but other solutions, e.g., cubic in $p$, are also possible; they are related to the dispersionless Korteweg-de Vries hierarchy [see Sec. II.C.4 and Okuyama and Takahashi (2016, 2017)].

Alternatively, one can construct a local dynamical invariant, or equivalently an extra potential to be added to the original Hamiltonian, to preserve the classical action for a fast time variation of the Hamiltonian parameters. Such an approach is reminiscent of the fast-forward method and can be solved implicitly as detailed by Jarzynski et al. (2017).

Finally, the counterdiabatic approach was also applied to classical spin dynamics (Hatomura and Mori, 2018). The construction makes use of the CD term for each single spin and is much easier to implement than in the corresponding quantum system. Moreover it does not need knowledge of the instantaneous stationary states. Starting from a stationary state of the initial Hamiltonian, it results in a stationary state of the final Hamiltonian if there is no criticality. The method can be used to solve combinatorial optimization problems.

\section{B. Mechanical engineering}

We discuss hereafter the interest of STA techniques to control a crane, then design robust solutions, and conclude with the link between STA and flatness based control in mathematics (Fliess et al., 1995).

\section{Cranes}

The objective of mechanical cranes is to move loads fast avoiding final pendulations and large sway angles en route that could compromise safety. Since a slow, adiabatic operation avoids excitations but it takes an impractical long time, cranes are a natural domain for STA (González-Resines et al., 2017). Crane control is an important engineering field; see Sun et al. (2012) and Kuo and Kang (2014) and references therein. Methods and ideas abound and many can be translated or adapted to other STA-driven processes in very different systems. For example, the work on closed-loop methods (in which measurements are performed en route to determine control operations) is an inspiring source to develop feedbackbased STA in the microscopic realm. In reverse, existing STA methods may have quite an impact on crane operation routines. Overhead cranes usually operate under a smalloscillation regime so that simple operations such as horizontal transport and hoistingor lowering of the load are modeled by the same basic (mass independent) equations that apply to the transport or compression or expansion of an ion in a timedependent harmonic trap. González-Resines et al. (2017) provided invariant-based STA protocols for the motion of the trolley in a transport operation or for hoisting that guarantee final adiabatic energies for the load. Furthermore, these energies are shown to be minimal when averaging over a microcanonical ensemble of initial conditions, consistently with the minimal work principle (Allahverdyan and Nieuwenhuizen, 2005, 2007). Indeed the possibility to design robust operations with respect to different perturbations or errors (such as dispersion in the initial conditions or in cable lengths) and STA and OCT combinations to limit, for example, en-route pendulations, offer a great potential. The different techniques to enhance robustness, such as the Fourier method [(Guéry-Odelin and Muga, 2014), see the next section], may be used to design trolley trajectories which are robust with respect to different errors, e.g., in the cable length. The bridge to perform analogous inverse engineering in quantum microscopic and classical macroscopic systems is much facilitated by the fact that the Lewis-Leach family of potentials implies the same classical (Lewis and Leach, 1982) and quantum formulations (Dhara and Lawande, 1984) for the invariants and auxiliary equations.

A crane model that treats the trolley position as a dynamical variable, instead of as a control function, subjected to inertia, the engine pulling force $\mathcal{F}_{a}$, dissipation, and the backaction of the load, is a neat, explicit test bed to study energy consumptions and the implications of shortcuts to adiabaticity from the point of view of the necessary external controls (Torrontegui et al., 2017). In particular, the power produced by the engine force is due to the change of mechanical energy $\mathcal{H}_{0}$ of the whole system (load and trolley) plus the power needed to compensate the effect of friction

$$
\mathcal{P}=\mathcal{F}_{a} \dot{x}=\frac{d \mathcal{H}_{0}}{d t}+\gamma \dot{x}^{2}
$$

where $x$ is the trolley's position and $\gamma$ the friction coefficient. In the harmonic approximation this becomes

$$
\mathcal{P}=\left(M \ddot{x}-m q \omega^{2}+\gamma \dot{x}\right) \dot{x}
$$

where $M$ is the trolley's mass and $q$ the load horizontal displacement with respect to the trolley; see Fig. 13. The second term is exactly the power $P$ defined as the derivative of the mechanical energy of the load. $\mathcal{P}=P$ under only rather extreme and even undesirable conditions.

Note that the shortcuts are by definition fast processes, so that the friction can hardly be avoided by the trick of slowing down the dynamics applied for ideal reversible operations. Only the typically unrealistic $\gamma=0$ scenario would cancel the dissipation term. As for the $M$-dependent term, the limit $M=0$ is again rather unrealistic, and in fact does not simplify matters, because the action of the engine would have to depend strongly on the initial conditions of the load; contrast this to ideal state-independent STA operations that require instead a large $M / m$ ratio. Another interesting aspect of the model is the analysis of possible negative values of $\mathcal{P}$ corresponding to braking. Different scenarios (implying energy consumption or rather partial regenerative braking) are depicted by Torrontegui et al. (2017) and treated phenomenologically to examine the total energy consumption.

We focused on cranes but clearly other mechanical machines and robots with moving parts can benefit from shortcuts to adiabaticity (Stefanatos, 2018), as well as other areas of engineering. For example, Faure et al. (2018) introduced inverse engineering to drive a resistor-capacitor (RC) circuit. 


\section{Robustness issues}

In Sec. II.A, we showed how the equation of motion of a particle in a moving harmonic potential of angular frequency $\omega_{0}$ can be reversed. We proposed to extend this technique using a Fourier method (Guéry-Odelin and Muga, 2014). From the Newton equation, one can directly relate the excess energy after the transport $\Delta E\left(t_{f}\right)=m\left|\mathcal{F}\left(\omega_{0} ;\left\{x_{0}(t)\right\}\right)\right|^{2}$ to the motion of the trap $x_{0}(t)$ with

$$
\mathcal{F}\left(\omega ;\left\{x_{0}(t)\right\}\right)=\int_{0}^{t_{f}} \ddot{x}_{0}\left(t^{\prime}\right) e^{-i \omega t^{\prime}} d t^{\prime}
$$

An optimal trajectory $\left\{\tilde{x}_{0}(t)\right\}$ for the transport shall therefore fulfill the relation $\mathcal{F}\left(\omega_{0} ;\left\{\tilde{x}_{0}(t)\right\}\right)=0$. A systematic way to generate such trajectories is to define the acceleration $\ddot{x}_{0}(t)$ through an auxiliary time-dependent function $g(t)$ as $\ddot{x}_{0}(t)=\ddot{g}(t)+\omega_{0}^{2} g(t)$, where $g(t)$ obeys the boundary conditions $g(0)=g\left(t_{f}\right)=\dot{g}(0)=\dot{g}\left(t_{f}\right)=0$ and the relations

$\int_{0}^{t_{f}} g(t) d t=0 \quad$ and $\quad \int_{0}^{t_{f}} d t^{\prime} \int_{0}^{t^{\prime}} g\left(t^{\prime \prime}\right) d t^{\prime \prime}=\frac{d}{\omega_{0}^{2}}$.

These conditions ensure that $x_{0}(0)=0, \quad \dot{x}_{0}(0)=0$, $x_{0}\left(t_{f}\right)=d$, and $\dot{x}_{0}\left(t_{f}\right)=0$. We then find

$$
\mathcal{F}\left(\omega ;\left\{x_{0}(t)\right\}\right)=\left(\omega_{0}^{2}-\omega^{2}\right) \int_{0}^{t_{f}} g\left(t^{\prime}\right) e^{-i \omega t^{\prime}} d t^{\prime},
$$

which vanishes for $\omega=\omega_{0}$, as expected for an optimal transport. Interestingly, using a fourth-order differential equation to relate $\ddot{x}_{0}$ and $g(t)$, and with appropriate boundary conditions for $g(t)$, one can factorize a polynomial in $\omega^{2}$ in front of the Fourier transform of $g(t)$ of the form $\left(\omega_{1}^{2}-\omega^{2}\right)\left(\omega_{2}^{2}-\omega^{2}\right)$ (Guéry-Odelin and Muga, 2014). With such a solution, the same trajectory of the trap would be optimal for two different angular frequencies $\omega_{1}$ and $\omega_{2}$. This would apply for instance for two different atoms transported by the same moving optical tweezers (Couvert et al., 2008). Alternatively, the protocol repeated for higher order polynomial with the same root $\omega_{0}$ provides a generic method to enforce robustness against the exact value of the angular frequency experienced by the atoms (Guéry-Odelin and Muga, 2014). For an application in ion transport, see An et al. (2016).

Flatness based control theory.-From a more mathematical point of view, the previous solution can also be recovered using flatness based control theory commonly used for steering a system from one state to another (Rouchon, 2005). This formalism applies to differential systems of the form

$$
d x / d t=f(x, u)
$$

where the vector $u$ contains the control variables. The control problem is readily solved when there exists a so-called flatoutput map $h, y(t)=h\left(x, u, \dot{u}, \ldots, u^{(\alpha)}\right)$, such that $x=$ $h_{1}\left(y, \dot{y}, \ldots, y^{(\beta)}\right)$ and $u=h_{2}\left(y, \dot{y}, \ldots, y^{(\beta+1)}\right)$, where $\alpha$ and $\beta$ are some finite numbers, and $h_{1}$ and $h_{2}$ some smooth functions. For a given system described by Eq. (134) there is no algorithm to determine if a flat-output map $h$ exists.
However, many examples of engineering interest turn out to be flat including transport (Rouchon, 2005) and cranes (Fliess et al., 1995).

\section{STA for isolated dilute gases}

So far we have essentially considered one-body problems. One may wonder to what extent the results can be generalized to an assembly of interacting atoms. We first consider a dilute gas trapped by a 3D isotropic harmonic potential of angular frequency $\omega_{0}$. The notion of adiabaticity shall be revisited in this new context. Indeed, the gas has a relaxation time $\tau$ that is related to the collision rate $\gamma_{c}=n \sigma \bar{v}$, where $n$ is the mean atomic density, $\sigma$ the total cross section, and $\bar{v} \propto\left(k_{B} T / m\right)^{1 / 2}$ the mean thermal velocity for a temperature $T$. The relation between $\tau$ and $\gamma_{c}$ depends on the collision regime: for $\gamma_{c} \ll \omega_{0}$, i.e., when there are few collisions per oscillation period $\tau \propto \gamma_{c}^{-1}$, while $\gamma_{c} \gg \omega_{0}$ in the hydrodynamic limit $\tau \propto \gamma_{c} / \omega_{0}^{2}$. The thermodynamical criterion for adiabaticity associated with a slow change of the angular frequency $\omega(t)$ now reads

$$
\frac{d \omega}{d t} \ll \frac{\omega}{\tau} .
$$

Once this criterion is fulfilled, the quantity $T(t) / \omega(t)$ remains constant. The physical interpretation of this conserved quantity is clear, it ensures that the populations $\pi_{n} \propto$ $\exp \left(-\hbar n \omega / k_{B} T\right)$ of the eigenstates are conserved during a slow change of confining strength. Otherwise stated, the transformation corresponds to a work and is not accompanied by heat (modifications of the populations).

\section{Boltzmann equation}

The search for a shortcut on such transformations requires a rigorous mathematical modeling of the out-of-equilibrium dynamics. Under the approximation of diluteness, the evolution of the phase-space distribution $f(\mathbf{r}, \mathbf{v}, t)$ of the gas is well described by the Boltzmann equation where collisions are accounted for through the two-body collisional integral. In Guéry-Odelin et al. (2014), an exact solution of this equation for a time-dependent angular frequency $\omega(t)$ was worked out,

$$
f(\mathbf{r}, \mathbf{v}, t)=\frac{\left(\alpha \beta-\gamma^{2} / 4\right)^{3 / 2}}{\pi^{3}} e^{-\alpha r^{2}-\beta v^{2}-\gamma \mathbf{r} \cdot \mathbf{v}},
$$

where $\alpha(t), \beta(t)$, and $\gamma(t)$ are functions that depend only on time. They are related through a linear set of differential equations which can be recast as a single third-order differential equation on the quantity $\beta$ which plays the role of the inverse of an effective temperature,

$$
\dddot{\beta}+4 \omega^{2} \dot{\beta}+4 \omega \dot{\omega} \beta=0
$$

For a slow transformation, the third-order derivative can be neglected and the quantity $T(t) / \omega(t) \propto[\beta(t) \omega(t)]^{-1}$ is conserved. Fast transformations can be designed by inverse engineering, i.e., by fixing the boundary conditions on $\beta$ and its derivatives, interpolating the $\beta$ function accordingly, 
and inferring $\omega(t)$ from the equation obeyed by $\beta$. For very fast decompression, we find as in quantum mechanics intervals of time over which the sign of the curvature of the potential is reversed. So far we have kept the trap isotropic. The extension of STA protocols to anisotropic 2D Bose gas and $3 \mathrm{D}$ unitary Fermi gases including in the presence of topological defects such as soliton or vortices was discussed by Papoular and Stringari (2015).

\section{Extension to Navier-Stokes equation}

The solution previously outlined hints at a related solution for hydrodynamics. Indeed, as originally demonstrated by Chapman and Enskog (Chapman, Cowling, and Burnet, 1970), the hydrodynamic equations can be derived from the Boltzmann equation. These equations relate the velocity field $\mathbf{v}(\mathbf{r}, t)$, the temperature field $T(\mathbf{r}, t)$, and the density $n(\mathbf{r}, t)$,

$$
\begin{aligned}
\frac{\partial n}{\partial t}+\boldsymbol{\nabla} \cdot(n \mathbf{v}) & =0 \\
m n\left(\frac{\partial}{\partial t}+\mathbf{v} \cdot \boldsymbol{\nabla}\right) \mathbf{u} & =-n \boldsymbol{\nabla} U-\boldsymbol{\nabla}\left(P-\frac{\eta}{3}(\boldsymbol{\nabla} \cdot \mathbf{v})\right)+\frac{\eta}{\rho} \nabla^{2} \mathbf{v} \\
\left(\frac{\partial}{\partial t}+\mathbf{v} \cdot \boldsymbol{\nabla}\right) T & =-\frac{T}{c_{V}}(\boldsymbol{\nabla} \cdot \mathbf{v})+\frac{\kappa \nabla^{2} T}{m n c_{V}}
\end{aligned}
$$

where $\eta$ is the viscosity, $c_{V}$ the specific heat, and $\kappa$ the thermal conductivity. The exact solution for a $3 \mathrm{D}$ isotropic harmonic trap with time-dependent angular frequency $\omega(t)$ is found through the search of an exact scaling solution of the form $n(\mathbf{r}, t)=b^{-3} n_{0}(\mathbf{r} / b), \mathbf{v}(\mathbf{r}, t)=\dot{b} \mathbf{r} / b$, and $T(\mathbf{r}, t)=\beta^{-1}(t)$. Remarkably, we found that the inverse of the effective temperature parameter $\beta$ and $\omega(t)$ are connected once again by Eq. (137). The same STA strategy can therefore be applied in this context.

\section{Shortcuts for systems in contact with a thermostat}

So far we have considered only isolated systems. In this section, we answered the question of how a classical system in contact with a thermal bath can benefit from an accelerated equilibration protocol. Indeed, the control in the presence of a thermostat is of general interest with applications ranging from nano-oscillators, nanothermal engines, to the driving of mesoscopic chemical or biological processes.

The one-body equation to be considered is therefore a stochastic differential equation, the Langevin equation, whose noise is related to the temperature of the bath. In its most general form, it is given by

$$
m \ddot{x}=-\partial_{x} U(x, t)-m \gamma \dot{x}+\xi(t),
$$

where we consider the noise $\xi(t)$ as a white noise delta correlated in time $\left\langle\xi(t) \xi\left(t^{\prime}\right)\right\rangle=2 m \gamma k_{B} T \delta\left(t-t^{\prime}\right)$. The inverse engineering method cannot be readily applied directly to a stochastic equation. Instead, the equation for the probability density $\rho(x, t)$ function associated with such a Brownian motion is used.

\section{The overdamped regime}

First we considered the overdamped regime for which inertial effects become negligible. This amounts to vanishing the mass in Eq. (139). The probability density then obeys the Fokker-Planck equation

$$
\partial_{t} \rho(x, t)=\gamma^{-1} \partial_{x}[\rho(x, t) U(x, t)]+D \partial_{x x}^{2} \rho,
$$

with the diffusion constant $D=k_{B} T / m \gamma$. Two standard STA methods can be applied to accelerate the equilibration: the transposition of the counterdiabatic ideas and the inverse engineering.

For the first method, we considered an equilibrium solution of Eq. (140), $\rho_{0}(x,\{\lambda\})$ that corresponds to the potential energy $U_{0}(x,\{\lambda\})$, where $\{\lambda\}$ refers to the control parameters of the potential. $U_{1}$ is the extra potential that we have to add to the original potential $U_{0}$ to compensate for the time variation of the parameters $\{\lambda\}$. This potential is the solution of the equation

$$
\partial_{t} \rho_{0}(x,\{\lambda(t)\})=\gamma^{-1} \partial_{x}\left[\rho_{0}(x,\{\lambda(t)\}) U_{1}(x, t)\right] .
$$

For instance, with a potential $U_{0}(x, t)=m \omega^{2}(t)\left[x-x_{0}(t)\right]^{2} / 2$, we find

$$
U_{1}(x, t)=-m \gamma \dot{x}_{0}\left[x-x_{0}(t)\right]+\frac{m \gamma \dot{\omega}\left[x-x_{0}(t)\right]^{2}}{2 \omega} .
$$

The potential $U_{1}(x, t)$ is nothing but the classical counterdiabatic Hamiltonian determined in Sec. VII.A (Li, Quan, and Tu, 2017).

The second strategy is to apply inverse engineering to the Fokker-Planck equation. For the compression or decompression of a harmonic oscillator from an angular frequency $\omega_{i}$ to $\omega_{f}$, we use an exact scaling solution of the overdamped Fokker-Planck equation (Martínez et al., 2016),

$$
\rho(x, t)=\sqrt{\frac{\alpha(t)}{\pi}} \exp \left[-\alpha(t) x^{2}\right]
$$

with

$$
\frac{\dot{\alpha}}{\alpha}=\frac{2 \omega^{2}(t)}{\gamma}-\frac{4 k_{B} T \alpha}{\gamma} .
$$

For our purpose, we imposed boundary conditions on the $\alpha$ parameter $\left[\alpha(0)=m \omega_{i}^{2} / 2 k_{B} T, \alpha\left(t_{f}\right)=m \omega_{f}^{2} / 2 k_{B} T, \dot{\alpha}(0)=0\right.$, and $\dot{\alpha}\left(t_{f}\right)=0$ ], interpolated the $\alpha(t)$ functions, and inferred from Eq. (144) how one shall shape $\omega(t)$. This solution was successfully implemented experimentally for a compression using a $1 \mu \mathrm{m}$ size microsphere trapped by an optical tweezer and immersed in a thermalized fluid chamber (Martínez et al., 2016). The strength of the confinement was simply increased by designing in time the intensity of the trap beam. In this manner, the system has reached equilibrium 100 times faster than the natural equilibration rate. Alternatively, the minimum time to perform transitions between thermal equilibrium states was also studied using optimal control theory (see Sec. II.G) under different constraints on the domain of variation of the time-dependent angular frequency $\omega(t)$ (Stefanatos, 2017b; Plata et al., 2019). 
As for the system described by the Boltzmann equation or for the quantum counterpart, the protocol requires for very fast decompression the transient use of a repulsive potential. In practice, this is arduous if not impossible depending on the system. However, and contrary to the quantum case, an extra parameter can be tuned in statistical physics, namely, the temperature. Indeed the temperature can be related to noise as explicitly written in the Langevin equation (Martínez et al., 2013). By a proper shaping of the noise, it is therefore possible to dramatically accelerate the decompression keeping the trap attractive as recently demonstrated experimentally (Chupeau, Besga et al., 2018).

\section{Connection with free energy and irreversible work}

A natural question arises: what is the work that can be extracted from a given transformation? In the context of an overdamped dynamics, this question was theoretically addressed by Sekimoto and Sasa (1997). The mean work $W$ done by the systems reads (Tu, 2014; Acconcia, Bonança, and Deffner, 2015; Martínez et al., 2016; Li, Quan, and Tu, 2017)

$$
W=\Delta F+W_{\text {irr }},
$$

where $\Delta F$ refers to the increment of the Helmholtz free energy associated with the transformation and $W_{\text {irr }}>0$ to the irreversible work. In the limit of slow variation of the control parameter the work done boils down to the variation of the Helmholtz free energy which becomes independent of the path used for the transformation. If $t_{f}$ refers to the interval over which the transformation is performed, one can show that the product $W_{\text {irr }} t_{f}$ is bounded in the limit $t_{f} \rightarrow \infty$. For a compression or decompression, we found $\Delta F=k_{B} T \log \left(\omega_{f} / \omega_{i}\right)$ and $W_{\text {irr }}=\eta k_{B} T\left(\omega_{f}^{2} / \omega_{i}^{2}\right) \tau_{\text {relax }} / t_{f}$, where the numerical factor $\eta$ depends upon the chosen protocol and $\tau_{\text {relax }}=\gamma / m \omega_{f}^{2}$.

\section{Extensions}

The extension of the previous approach to the underdamped regime was worked out by Cunuder et al. (2016), Li, Quan, and Tu (2017), and Chupeau, Ciliberto et al. (2018). The inverse engineering approach can be readily generalized to manipulate the phase-space distribution $\rho(x, v, t)$ (Chupeau, Ciliberto et al., 2018). This technique was used experimentally to accelerate the equilibration of a micromechanical oscillator (Cunuder et al., 2016). However, the transposition of the counterdiabatic method provides an auxiliary potential linear in $p$ that is not relevant from an experimental point of view (Li, Quan, and Tu, 2017).

\section{OUTLOOK AND OPEN QUESTIONS}

Since the birth of the term in 2010 until today, shortcuts to adiabaticity have experienced phenomenal growth. ${ }^{15}$

\footnotetext{
${ }^{15}$ A Web-of-Science search including the main keywords gives 7 citations in 2010, 359 in 2014, and 1543 in 2018, with an $h$ index of $\sim 40$.
}

We attribute this expansion to a double appeal, both practical and fundamental:

The practical side is rooted in the fact that adiabatic invariance is ubiquitous as a phenomenon and as a route for state preparation, in quantum physics and beyond. Shortcuts overcome adiabatic protocols, which imply long times and the concomitant accumulation of perturbations from the environment or the control system. Moreover a rich network of different pure or hybrid STA approaches provides a flexible toolbox that can be applied and adapted to many systems and operations. Only in the quantum arena proposals exist for STA-mediated cooling, interferometers, photon production, enantiomer separation, quantum gates, or information transfer. Methodological progress in one area can be translated to others allowing for synergies. Quantum physics has been indeed the main field to develop STA methodology and applications so far. As the control of microscopic systems improves, we get closer to realizing new quantum technologies. Yet, decoherence remains a stumbling block to go beyond proof-of-principle results. Shortcuts contribute to fight decoherence via shorter process times and robust protocols. An ideal quantum device is expected to operate fast and accurately despite a noisy environment or perturbations and with minimal consumption of resources. These are all goals that fit into the agenda and capabilities of STA methods.

The fundamental side rests on the fact that basic concepts and physical quantities and phenomena such as robustness, timing, energy and work, information, entropy, needed resources, controllability, environment effects, or classical or quantum borders and connections all play a role in shortcut design, so shortcuts motivate and contribute to the quest for their interrelations. Early work on the time-dependent harmonic oscillator made clear that the process time and timeaveraged energies involved in the STA-driven process implied nontrivial inequalities and speed limits that helped to quantify cooling speeds. The scrutiny of energy-time relations has grown in different directions, e.g., to analyze energy costs and their scaling with process times. The fast nature of STA processes makes some conventional estimates based on slow reversible processes invalid. As well, fundamental questions on the meaning and appropriate definitions of work, heat, or efficiencies arise. The counterdiabatic Hamiltonian bridges the gap between actual and ideal transitionless dynamics and so it enters in a number of fundamental inequalities to set speed limits, see Sec. IV, but also as an aid to reach maximal precision limits as discussed in Sec. III.H. Recent work demonstrates energy and time relations may be quite rich for open systems bringing to the fore further elements such as entropy production, robustness, and/or information erasure (Takahashi, 2017a; Boyd et al., 2018; Funo, Shiraishi, and Saito, 2019).

In this review we have seen a number of problem- or fieldspecific challenges. Here we underline a few open questions we consider to be important along broader conceptual or methodological lines.

- We have discussed examples for which the full spectral information of the original Hamiltonian is not necessary to perform the shortcuts. As well, approximate schemes are being developed. Improving approximations and 
spectral-information independence are of upmost importance for complex quantum systems such as many-body systems, multiple levels, or for adiabatic computing. The question of the minimum required information for a given transformation that needs to be accelerated has not been investigated systematically so far.

- We have discussed the key role played by dynamical invariants and their link with other STA techniques. Open questions are (a) to find further families of Hamiltonianinvariant pairs beyond the Lewis-Leach family, e.g., via Lax pairs; (b) in $n$-dimensional systems, to find and implement effective schemes when the dynamical normal modes need a generalized transformation (involving coordinates and momenta); and (c) to explore the use for STA design of different generalizations of "invariant" operators for nonHermitian systems.

- Steering a dynamical quantum system from an initial to a final state in the presence of an environment poses a challenge for quantum control on controllability, i.e., to which extent the target state is reachable; and control design, possibly including reservoir engineering. A framework to draw the frontiers of controllability is highly desirable for quantum control in open systems (Glaser et al., 2015; Koch, 2016), but note that shortcuts may be applied even if the system is not fully controllable (Petiziol et al., 2018).

- We have provided examples of hybrid control approaches, for instance, hybridation of shortcuts and optimal control theory that allows for optimal protocol selection. From a control perspective, shortcuts to adiabaticity have contributed to open-loop (with no-feedback) design. One could also envision the hybridation of such feed-forward techniques with feedback oriented techniques. Indeed, STA could help to approach the target very fast and the final convergence could be ensured by a feedback procedure. Such a strategy would benefit from the advantages of both techniques: short time processing and strong robustness.

- We need to clarify the energetic and resource cost of STA approaches, and the associated trade-off relations. This is of general interest both at the fundamental level and for specific experiments, for example, to determine the actual performance of STA-enhanced microscopic or mesoscopic engines and refrigerators.

- STA protocols can a priori be adapted to a large class of other dynamical or differential equations, e.g., in engineering, plasma physics, optics, soft condensed matter, or biology.

\section{LIST OF SYMBOLS AND ABBREVIATIONS}

1D, 2D, etc. one dimensional, two dimensional, etc. $\mathrm{BEC}$

$\mathrm{CD}$

CNOT

CS

dc

DFS

DRAG

FAQUAD

$\mathrm{FF}$

$\begin{array}{ll}\text { GHZ } & \text { Greenberger-Horne-Zeilinger } \\ \text { IE } & \text { inverse engineering } \\ \text { IP } & \text { interaction picture } \\ \text { NV } & \text { nitrogen vacancy } \\ \text { OCT } & \text { optimal control theory } \\ \text { PDE } & \text { partial differential equation } \\ \text { PS } & \text { primary system } \\ \text { QED } & \text { quantum electrodynamics } \\ \text { QZD } & \text { quantum Zeno dynamics } \\ \text { STA } & \text { shortcut to adiabaticity } \\ \text { STIRAP } & \text { stimulated Raman adiabatic passage } \\ \text { SVEA } & \text { slowly varying envelope approximation } \\ \text { WAHWAH } & \text { weak anharmonicity with average } \\ & \text { Hamiltonian }\end{array}$

\section{ACKNOWLEDGMENTS}

We thank P. Claeys, S. Deffner, C. Jarzynski, R. Kosloff, M. Sarandy, E. Sherman, D. Sugny, K. Takahashi, E. Trizac, and S.-Y. Tseng for clarifying comments or a critical reading of the manuscript or sections of it. Many colleagues and collaborators, too numerous to mention individually, have contributed through the last 10 years to our work on shortcuts. We are deeply indebted to all of them. This work was supported by the Basque Country Government (Grant No. IT986-16); PGC2018-101355-B-100 (MCIU/AEI/FEDER, UE); PGC2018-094792-B-100 (MCIU/AEI/FEDER, EU); CAM/ FEDER Project No. S2018/TCS-4342 (QUITEMAD-CM); and by Programme Investissements d'Avenir under the Grant ANR-11-IDEX-0002-02, reference ANR-10-LABX0037-NEXT, as well as the Grant ANR-18-CE30-0013.

\section{APPENDIX A: EXAMPLE OF LIE TRANSFORM}

As an example to illustrate the use of "physical" unitary transformations to generate alternative shortcuts we considered a two-level system in which we applied a Lie transform to get rid of a $\sigma_{y}$ term in the Hamiltonian found with the CD approach. The reference Hamiltonian $H_{0}$ is given in Eq. (15) and $H_{C D}$ in Eq. (16). The generators of the dynamical algebra are the Pauli matrices,

$G_{1}=\left(\begin{array}{cc}0 & 1 \\ 1 & 0\end{array}\right), \quad G_{2}=\left(\begin{array}{cc}0 & -i \\ i & 0\end{array}\right), \quad G_{3}=\left(\begin{array}{cc}1 & 0 \\ 0 & -1\end{array}\right)$,

which satisfy the commutation relations $\left[G_{a}, G_{b}\right]=2 i \epsilon_{a b c} G_{c}$. The total Hamiltonian $H(t)=H_{0}(t)+H_{C D}(t)$ in terms of the algebra generators can be written as

$$
H(t)=\frac{\hbar}{2}\left[\Omega_{R}(t) G_{1}-\Delta(t) G_{3}\right]+\frac{\hbar}{2} \Omega_{a}(t) G_{2} .
$$

Suppose that the generator $G_{2}$ is difficult or inconvenient to implement; see, for example, Bason et al. (2012). Setting $G=G_{3}$ in Eq. (29), and substituting into Eq. (30), the series of repeated commutators may be summed up. $H^{\prime}$ becomes 
D. Guéry-Odelin et al.: Shortcuts to adiabaticity: Concepts, methods, ...

$$
\begin{aligned}
H^{\prime}(t)= & \frac{\hbar}{2}\left\{\left[\Omega_{R}(t) \cos (2 g(t))+\Omega_{a}(t) \sin (2 g(t))\right] G_{1}\right. \\
& -\left[\Omega_{R}(t) \sin (2 g(t))-\Omega_{a}(t) \cos (2 g(t))\right] G_{2} \\
& \left.-[\Delta(t)+2 \dot{g}(t)] G_{3}\right\} .
\end{aligned}
$$

To cancel the $G_{2}$ term, we choose

$$
g(t)=\frac{1}{2} \arctan \left[\frac{\Omega_{a}(t)}{\Omega_{R}(t)}\right] .
$$

Substituting Eq. (A4) into Eq. (A3) we have finally

$$
\begin{aligned}
H^{\prime}(t)= & \frac{\hbar}{2}\left\{\left[\sqrt{1+\frac{\Omega_{a}^{2}(t)}{\Omega_{R}^{2}(t)}} \Omega_{R}(t)\right] G_{1}\right. \\
& \left.-\left[\Delta+\frac{\Omega_{R}(t) \dot{\Omega}_{a}(t)-\dot{\Omega}_{R}(t) \Omega_{a}(t)}{\Omega_{R}^{2}(t)+\Omega_{a}^{2}(t)}\right] G_{3}\right\},
\end{aligned}
$$

which has the same structure (generators) as the reference Hamiltonian (15) but different time-dependent coefficients. A similar result can be found without starting from the reference shortcut (16), but following the bottom-up approach of Torrontegui, Martínez-Garaot, and Muga (2014); see Sec. II.C.1.

\section{APPENDIX B: COUNTERDIABATIC HAMILTONIAN FOR A TWO-LEVEL HAMILTONIAN WITH COMPLEX COUPLING}

We found here $H_{C D}$ for a two-level Hamiltonian $H_{0}$ with complex-valued coupling $\Omega$,

$$
H_{0}(t)=\frac{\hbar}{2}\left(\begin{array}{cc}
-\Delta(t) & |\Omega(t)| e^{i \alpha(t)} \\
|\Omega(t)| e^{-i \alpha(t)} & \Delta(t)
\end{array}\right),
$$

where $|\Omega(t)|$ is the modulus and $\alpha(t)$ the argument of the coupling. The instantaneous eigenvectors of this Hamiltonian are

$$
\begin{aligned}
& \left|\lambda_{-}(t)\right\rangle=-\sin [\theta(t) / 2] e^{i \alpha(t) / 2}|1\rangle+\cos [\theta(t) / 2] e^{-i \alpha(t) / 2}|2\rangle \\
& \left|\lambda_{+}(t)\right\rangle=\cos [\theta(t) / 2] e^{i \alpha(t) / 2}|1\rangle+\sin [\theta(t) / 2] e^{-i \alpha(t) / 2}|2\rangle
\end{aligned}
$$

with the mixing angle $\theta(t) \equiv \arccos [-\Delta(t) / \tilde{\Omega}(t)]$ and eigenvalues $E_{\mp}(t)=\mp \hbar \tilde{\Omega} / 2$, where $\tilde{\Omega}=\sqrt{\Delta^{2}(t)+|\Omega(t)|^{2}}$. The counterdiabatic Hamiltonian is given by $H_{C D}=$ $H_{C D}^{(1)}+H_{C D}^{(2)}$, where $H_{C D}^{(1)}=i \hbar \sum_{n}|\dot{n}(t)\rangle\langle n(t)|$ and $H_{C D}^{(2)}=$ $-i \hbar \sum_{n}\langle n(t) \mid \dot{n}(t)\rangle|n(t)\rangle\langle n(t)|$. For the Hamiltonian $H_{0}(t)$ in Eq. (B1), we get

$$
H_{C D}^{(1)}(t)=\frac{\hbar}{2}\left(\begin{array}{cc}
-\dot{\alpha}(t) & -i e^{i \alpha(t)} \dot{\theta}(t) \\
i e^{-i \alpha(t)} \dot{\theta}(t) & \dot{\alpha}(t)
\end{array}\right)
$$

and

$$
\begin{aligned}
H_{C D}^{(2)}(t)= & \frac{\hbar}{2} \cos \theta(t) \dot{\alpha}(t) \\
& \times\left(\begin{array}{cc}
\cos \theta(t) & e^{i \alpha(t)} \sin \theta(t) \\
e^{-i \alpha(t)} \sin \theta(t) & -\cos \theta(t)
\end{array}\right) .
\end{aligned}
$$

If $\alpha(t)=0$, we have that $H_{C D}^{(2)}=0$, and the expression of $H_{C D}^{(1)}(t)$ simplifies to the one given, e.g., in Eq. (16) or Chen, Lizuain et al. (2010).

\section{REFERENCES}

Abah, O., and E. Lutz, 2016, Europhys. Lett. 113, 60002.

Abah, O., and E. Lutz, 2017, Europhys. Lett. 118, 40005.

Abah, O., and E. Lutz, 2018, Phys. Rev. E 98, 032121.

Abah, O., and M. Paternostro, 2018, arXiv:1808.00580.

Acconcia, T. V., M. V. S. Bonança, and S. Deffner, 2015, Phys. Rev. E 92, 042148.

Agundez, R. R., C. D. Hill, L. C. L. Hollenberg, S. Rogge, and M. Blaauboer, 2017, Phys. Rev. A 95, 012317.

Albash, T., S. Boixo, D. A. Lidar, and P. Zanardi, 2012, New J. Phys. 14, 123016.

Albash, T., S. Boixo, D. A. Lidar, and P. Zanardi, 2015, New J. Phys. 17, 129501.

Albash, T., and D. A. Lidar, 2018, Rev. Mod. Phys. 90, 015002.

Allahverdyan, A. E., and T. M. Nieuwenhuizen, 2005, Phys. Rev. E 71, 046107.

Allahverdyan, A. E., and T. M. Nieuwenhuizen, 2007, Phys. Rev. E 75, 051124.

Alonso, J., F. M. Leupold, Z. U. Solèr, M. Fadel, M. Marinelli, B. C. Keitch, V. Negnevitsky, and J. P. Home, 2016, Nat. Commun. 7, 11243.

Amezcua, M., A. Golter, and H. Wang, 2017, in 2017 Conference on Lasers and Electro-Optics (CLEO) (Optical Society of America, Washington, DC), pp. 1-2.

Amin, M. H. S., 2008, Phys. Rev. Lett. 100, 130503.

Amri, S., R. Corgier, D. Sugny, E. Rasel, N. Gaaloul, and E. Charron, 2018, arXiv:1812.10999.

An, S., D. Lv, A. del Campo, and K. Kim, 2016, Nat. Commun. 7, 12999.

Anandan, J., and Y. Aharonov, 1990, Phys. Rev. Lett. 65, 1697.

Babajanova, G., J. Matrasulov, and K. Nakamura, 2018, Phys. Rev. E 97, 042104.

Bachmann, S., W. De Roeck, and M. Fraas, 2017, Phys. Rev. Lett. 119, 060201.

Baksic, A., R. Belyansky, H. Ribeiro, and A. A. Clerk, 2017, Phys. Rev. A 96, 021801.

Baksic, A., H. Ribeiro, and A. A. Clerk, 2016, Phys. Rev. Lett. 116, 230503.

Ban, Y., and X. Chen, 2014, Sci. Rep. 4, 6258.

Ban, Y., X. Chen, S. Kohler, and G. Platero, 2019, arXiv:1904.05694.

Ban, Y., X. Chen, J. G. Muga, and E. Y. Sherman, 2015, Phys. Rev. A 91, 023604.

Ban, Y., X. Chen, E. Y. Sherman, and J. G. Muga, 2012a, Phys. Rev. Lett. 109, 249901.

Ban, Y., X. Chen, E. Y. Sherman, and J. G. Muga, 2012b, Phys. Rev. Lett. 109, 206602.

Bason, M. G., M. Viteau, N. Malossi, P. Huillery, E. Arimondo, D. Ciampini, R. Fazio, V. Giovannetti, R. Mannella, and O. Morsch, 2012, Nat. Phys. 8, 147.

Beau, M., J. Jaramillo, and A. del Campo, 2016, Entropy 18, 168. 
Benseny, A., A. Kiely, Y. Zhang, T. Busch, and A. Ruschhaupt, 2017, EPJ Quantum Techno. 4, 3.

Bernardo, B. d. L., 2019, arXiv:1907.01916.

Berry, M. V., 1987, Proc. R. Soc. A 414, 31.

Berry, M. V., 2009, J. Phys. A 42, 365303.

Boldt, F., P. Salamon, and K. H. Hoffmann, 2016, J. Phys. Chem. A 120, 3218.

Bowler, R., J. Gaebler, Y. Lin, T. R. Tan, D. Hanneke, J. D. Jost, J. P. Home, D. Leibfried, and D. J. Wineland, 2012, Phys. Rev. Lett. 109, 080502.

Boyd, A. B., A. Patra, C. Jarzynski, and J. P. Crutchfield, 2018, arXiv:1812.11241.

Boyers, E., M. Pandey, D. K. Campbell, A. Polkovnikov, D. Sels, and A. O. Sushkov, 2018, arXiv:1811.09762.

Bravetti, A., and D. Tapias, 2017, Phys. Rev. E 96, 052107.

Bücker, R., T. Berrada, S. Van Frank, J.-F. Schaff, T. Schumm, J. Schmiedmayer, G. Jäger, J. Grond, and U. Hohenester, 2013, J. Phys. B 46, 104012.

Bücker, R., J. Grond, S. Manz, T. Berrada, T. Betz, C. Koller, U. Hohenester, T. Schumm, A. Perrin, and J. Schmiedmayer, 2011, Nat. Phys. 7, 608.

Bukov, M., D. Sels, and A. Polkovnikov, 2019, Phys. Rev. X 9 , 011034.

Čadež, T., J. Jefferson, and A. Ramšak, 2013, New J. Phys. 15, 013029.

Čadež, T., J. H. Jefferson, and A. Ramšak 2014, Phys. Rev. Lett. 112, 150402.

Çakmak, B., and Ö. E. Müstecaplıŏglu, 2018, arXiv:1812.00969.

Calzetta, E., 2018, Phys. Rev. A 98, 032107.

Campbell, S., G. De Chiara, M. Paternostro, G. M. Palma, and R. Fazio, 2015, Phys. Rev. Lett. 114, 177206.

Campbell, S., and S. Deffner, 2017, Phys. Rev. Lett. 118, 100601.

Campisi, M., P. Hänggi, and P. Talkner, 2011, Rev. Mod. Phys. 83, 771.

Castin, Y., and R. Dum, 1996, Phys. Rev. Lett. 77, 5315.

Chapman, S., T. G. Cowling, and D. Burnet, 1970, The Mathematical Theory of Non-Uniform Gases: An Account of the Kinetic Theory of Viscosity, Thermal Conduction, and Diffusion in Gases (Cambridge University Press, Cambridge, England).

Chen, J., and L. F. Wei, 2015, Phys. Rev. A 91, 023405.

Chen, X., R.-L. Jiang, J. Li, Y. Ban, and E. Y. Sherman, 2018, Phys. Rev. A 97, 013631.

Chen, X., I. Lizuain, A. Ruschhaupt, D. Guéry-Odelin, and J. G. Muga, 2010, Phys. Rev. Lett. 105, 123003.

Chen, X., and J. G. Muga, 2010, Phys. Rev. A 82, 053403.

Chen, X., and J. G. Muga, 2012, Phys. Rev. A 86, 033405.

Chen, X., A. Ruschhaupt, S. Schmidt, A. del Campo, D. GuéryOdelin, and J. G. Muga, 2010, Phys. Rev. Lett. 104, 063002.

Chen, X., E. Torrontegui, and J. G. Muga, 2011, Phys. Rev. A 83, 062116.

Chen, X., E. Torrontegui, D. Stefanatos, J.-S. Li, and J. G. Muga, 2011, Phys. Rev. A 84, 043415.

Chen, X., H.-W. Wang, Y. Ban, and Shuo-Yen, 2014, Opt. Express 22, 24169.

Chen, X., R.-D. Wen, J.-L. Shi, and S.-Y. Tseng, 2018, J. Opt. 20, 045804.

Chen, X., R.-D. Wen, and S.-Y. Tseng, 2016, Opt. Express 24, 18322. Chen, Y.-H., B.-H. Huang, J. Song, and Y. Xia, 2016, Opt. Commun. 380, 140.

Chen, Y.-H., Z.-C. Shi, J. Song, and Y. Xia, 2018, Phys. Rev. A 97, 023841.

Chen, Y.-H., Z.-C. Shi, J. Song, Y. Xia, and S.-B. Zheng, 2017, Phys. Rev. A 95, 062319.
Chen, Y.-H., Q.-C. Wu, B.-H. Huang, J. Song, Y. Xia, and S.-B. Zheng, 2018, Ann. Phys. (Berlin) 530, 1700247.

Chen, Y.-H., Y. Xia, Q.-Q. Chen, and J. Song, 2014a, Phys. Rev. A 89, 033856.

Chen, Y.-H., Y. Xia, Q.-Q. Chen, and J. Song, 2014b, Laser Phys. Lett. 11, 115201.

Chen, Y.-H., Y. Xia, Q.-Q. Chen, and J. Song, 2015, Phys. Rev. A 91, 012325.

Chen, Y.-H., Y. Xia, J. Song, and Q.-Q. Chen, 2015, Sci. Rep. 5, 15616.

Chen, Y.-H., Y. Xia, Q.-C. Wu, B.-H. Huang, and J. Song, 2016, Phys. Rev. A 93, 052109.

Chen, Z., Y.-H. Chen, Y. Xia, J. Song, and B.-H. Huang, 2016, Sci. Rep. 6, 22202.

Choi, S., R. Onofrio, and B. Sundaram, 2011, Phys. Rev. A 84, 051601.

Choi, S., R. Onofrio, and B. Sundaram, 2012, Phys. Rev. A 86, 043436.

Chotorlishvili, L., M. Azimi, S. Stagraczyński, Z. Toklikishvili, M. Schüler, and J. Berakdar, 2016, Phys. Rev. E 94, 032116.

Chung, H.-C., K.-S. Lee, and S.-Y. Tseng, 2017, Opt. Express 25, 13626.

Chung, H.-C., and S.-Y. Tseng, 2018, Opt. Express 26, 9655.

Chupeau, M., B. Besga, D. Guéry-Odelin, E. Trizac, A. Petrosyan, and S. Ciliberto, 2018, Phys. Rev. E 98, 010104.

Chupeau, M., S. Ciliberto, D. Guéry-Odelin, and E. Trizac, 2018, arXiv:1802.10512.

Claeys, P. W., M. Pandey, D. Sels, and A. Polkovnikov, 2019, arXiv: 1904.03209.

Cohn, J., A. Safavi-Naini, R. J. Lewis-Swan, J. G. Bohnet, M. Gärttner, K. A. Gilmore, J. E. Jordan, A. M. Rey, J. J. Bollinger, and J. K. Freericks, 2018, New J. Phys. 20, 055013.

Corgier, R., S. Amri, W. Herr, H. Ahlers, J. Rudolph, D. GuéryOdelin, E. M. Rasel, E. Charron, and N. Gaaloul, 2018, New J. Phys. 20, 055002.

Coto, R., V. Jacques, G. Hétet, and J. R. Maze, 2017, Phys. Rev. B 96, 085420.

Coulamy, I. B., A. C. Santos, I. Hen, and M. S. Sarandy, 2016, Front. ICT 3, 19.

Couvert, A., T. Kawalec, G. Reinaudi, and D. Guéry-Odelin, 2008, Europhys. Lett. 83, 13001.

Cui, Y.-Y., X. Chen, and J. G. Muga, 2016, J. Phys. Chem. A 120, 2962.

Cunuder, A. L., I. A. Martínez, A. Petrosyan, D. Guéry-Odelin, E. Trizac, and S. Ciliberto, 2016, Appl. Phys. Lett. 109, 113502.

Daems, D., A. Ruschhaupt, D. Sugny, and S. Gurin, 2013, Phys. Rev. Lett. 111, 050404.

Damski, B., 2014, J. Stat. Mech. 12, P12019.

Damski, B., 2015, Sci. Rep. 5, 15779.

Dann, R., A. Levy, and R. Kosloff, 2018, Phys. Rev. A 98, 052129.

Dann, R., A. Tobalina, and R. Kosloff, 2018, arXiv:1812.08821.

Deffner, S., 2016, New J. Phys. 18, 012001.

Deffner, S., and S. Campbell, 2017, J. Phys. A 50, 453001.

Deffner, S., C. Jarzynski, and A. del Campo, 2014, Phys. Rev. X 4, 021013.

Deffner, S., and E. Lutz, 2013a, J. Phys. A 46, 335302.

Deffner, S., and E. Lutz, 2013b, Phys. Rev. Lett. 111, 010402.

del Campo, A., 2011, Phys. Rev. A 84, 031606.

del Campo, A., 2013, Phys. Rev. Lett. 111, 100502.

del Campo, A., and M. G. Boshier, 2012, Sci. Rep. 2, 648.

del Campo, A., J. Goold, and M. Paternostro, 2014, Sci. Rep. 4, 6208.

del Campo, A., and K. Kim, 2019, arXiv:1902.06283. 
del Campo, A., M. M. Rams, and W. H. Zurek, 2012, Phys. Rev. Lett. 109, 115703.

del Campo, A., and K. Sengupta, 2015, Eur. Phys. J. Spec. Top. 224, 189.

Della Valle, G., 2018, Phys. Rev. A 98, 053861.

Della Valle, G., G. Perozziello, and S. Longhi, 2016, J. Opt. 18, 09LT03.

Demirplak, M., and S. A. Rice, 2003, J. Phys. Chem. A 107, 9937. Demirplak, M., and S. A. Rice, 2005, J. Phys. Chem. B 109, 6838. Demirplak, M., and S. A. Rice, 2008, J. Chem. Phys. 129, 15411. Deng, J., Q.-h. Wang, Z. Liu, P. Hänggi, and J. Gong, 2013, Phys. Rev. E 88, 062122.

Deng, S., A. Chenu, P. Diao, F. Li, S. Yu, I. Coulamy, A. del Campo, and $\mathrm{H}$. Wu, 2018, Sci. Adv. 4, eaar5909.

Deng, S., P. Diao, Q. Yu, A. del Campo, and H. Wu, 2018, Phys. Rev. A 97, 013628.

Dhara, A. K., and S. V. Lawande, 1984, J. Phys. A 17, 2423.

Diffo, J., M. Ateuafack, G. Fouokeng, L. Fai, and M. Tchoffo, 2017, Superlattices Microstruct. 111, 310.

Dorner, U., P. Fedichev, D. Jaksch, M. Lewenstein, and P. Zoller, 2003, Phys. Rev. Lett. 91, 073601.

Dowdall, T., A. Benseny, T. Busch, and A. Ruschhaupt, 2017, Phys. Rev. A 96, 043601.

Du, Y.-X., Z.-T. Liang, Y.-C. Li, X.-X. Yue, Q.-X. Lv, W. Huang, X. Chen, H. Yan, and S.-L. Zhu, 2016, Nat. Commun. 7, 12479.

Du, Y.-X., B.-J. Liu, Q.-X. Lv, X.-D. Zhang, H. Yan, and S.-L. Zhu, 2017, Phys. Rev. A 96, 012333.

Duncan, C. W., and A. del Campo, 2018, New J. Phys. 20, 085003. Dupont-Nivet, M., C. I. Westbrook, and S. Schwartz, 2016, New J. Phys. 18, 113012.

Emmanouilidou, A., X.-G. Zhao, P. Ao, and Q. Niu, 2000, Phys. Rev. Lett. 85, 1626.

Ermakov, V. P., 1880, Universita Izvestia Kiev, Series III 9, 1.

Facchi, P., and S. Pascazio, 2002, Phys. Rev. Lett. 89, 080401.

Faure, S., S. Ciliberto, E. Trizac, and D. Guéry-Odelin, 2018, arXiv: 1808.01321.

Fliess, M., J. Lévine, P. Martin, and P. Rouchon, 1995, International Journal of Control 61, 1327.

Fogarty, T., L. Ruks, J. Li, and T. Busch, 2019, SciPost Phys. 6, 21.

Funo, K., N. Lambert, B. Karimi, J. Pekola, Y. Masuyama, and F. Nori, 2019, arXiv:1905.03480.

Funo, K., N. Shiraishi, and K. Saito, 2019, New J. Phys. 21, 013006.

Funo, K., J.-N. Zhang, C. Chatou, K. Kim, M. Ueda, and A. del Campo, 2017, Phys. Rev. Lett. 118, 100602.

Fürst, H. A., M. H. Goerz, U. G. Poschinger, M. Murphy, S. Montangero, T. Calarco, F. Schmidt-Kaler, K. Singer, and C. P. Koch, 2014, New J. Phys. 16, 075007.

Gao, X.-C., J.-B. Xu, and T.-Z. Qian, 1992, Phys. Rev. A 46, 3626. Garrido, L., 1964, J. Math. Phys. (N.Y.) 5, 355.

Giannelli, L., and E. Arimondo, 2014, Phys. Rev. A 89, 033419.

Glaser, S. J., et al., 2015, Eur. Phys. J. D 69, 279.

Goldstein, H., C. Poole, and J. Safko, 2002, Classical Mechanics (Pearson, Hoboken).

González-Resines, S., D. Guéry-Odelin, A. Tobalina, I. Lizuain, E. Torrontegui, and J. G. Muga, 2017, Phys. Rev. Applied 8, 054008.

Gritsev, V., P. Barmettler, and E. Demler, 2010, New J. Phys. 12, 113005.

Guérin, S., V. Hakobyan, and H. R. Jauslin, 2011, Phys. Rev. A 84, 013423.

Guérin, S., S. Thomas, and H. R. Jauslin, 2002, Phys. Rev. A 65, 023409.

Guéry-Odelin, D., and J. G. Muga, 2014, Phys. Rev. A 90, 063425.
Guéry-Odelin, D., J. G. Muga, M. J. Ruiz-Montero, and E. Trizac, 2014, Phys. Rev. Lett. 112, 180602.

Güngördü, U., Y. Wan, M. A. Fasihi, and M. Nakahara, 2012, Phys. Rev. A 86, 062312.

Guo, D., and T. Chu, 2017, Opt. Express 25, 9160.

Hartmann, A., and W. Lechner, 2018, arXiv:1807.02053.

Hatomura, T., 2017, J. Phys. Soc. Jpn. 86, 094002.

Hatomura, T., 2018, New J. Phys. 20, 015010.

Hatomura, T., and T. Mori, 2018, arXiv:1806.00229.

Hatomura, T., and K. Pawłowski, 2019, arXiv:1901.03519.

He, S., S.-L. Su, D.-Y. Wang, W.-M. Sun, C.-H. Bai, A.-D. Zhu, H.-F. Wang, and S. Zhang, 2016, Sci. Rep. 6, 30929.

Hegerfeldt, G. C., 2013, Phys. Rev. Lett. 111, 260501.

Herrera, M., M. S. Sarandy, E. I. Duzzioni, and R. M. Serra, 2014, Phys. Rev. A 89, 022323.

Ho, C.-P., and S.-Y. Tseng, 2015, Opt. Lett. 40, 4831.

Hoffmann, K. H., P. Salamon, Y. Rezek, and R. Kosloff, 2011, Europhys. Lett. 96, 60015.

Hollenberg, L. C. L., 2012, Nat. Phys. 8, 113.

Home, J. P., and A. M. Steane, 2005, arXiv:quant-ph/0411102.

Horowitz, J. M., and K. Jacobs, 2015, Phys. Rev. Lett. 115, 130501.

Hu, C.-K., J.-M. Cui, A. C. Santos, Y.-F. Huang, M. S. Sarandy, C.-F. Li, and G.-C. Guo, 2018, Opt. Lett. 43, 3136.

Huang, B.-H., Y.-H. Chen, Q.-C. Wu, J. Song, and Y. Xia, 2016, Laser Phys. Lett. 13, 105202.

Huang, W., L. Ang, and E. Kyoseva, 2017, arXiv:1711.05507.

Huang, X.-B., Y.-H. Chen, and Z. Wang, 2016a, Sci. Rep. 6, 25707.

Huang, X.-B., Y.-H. Chen, and Z. Wang, 2016b, Eur. Phys. J. D 70, 171.

Huang, X.-B., Z.-R. Zhong, and Y.-H. Chen, 2015, Quantum Inf. Process. 14, 4475.

Hung, Y.-J., Z.-Y. Li, H.-C. Chung, F.-C. Liang, M.-Y. Jung, T.-H. Yen, and S.-Y. Tseng, 2019, Opt. Lett. 44, 815.

Ibáñez, S., X. Chen, and J. G. Muga, 2013, Phys. Rev. A 87, 043402. Ibáñez, S., X. Chen, E. Torrontegui, J. G. Muga, and A. Ruschhaupt, 2012, Phys. Rev. Lett. 109, 100403.

Ibáñez, S., Y.-C. Li, X. Chen, and J. G. Muga, 2015, Phys. Rev. A 92 , 062136

Ibáñez, S., S. Martínez-Garaot, X. Chen, E. Torrontegui, and J. G. Muga, 2011, Phys. Rev. A 84, 023415.

Ibáñez, S., S. Martínez-Garaot, X. Chen, E. Torrontegui, and J. G. Muga, 2012, Phys. Rev. A 86, 019901.

Ibáñez, S., and J. G. Muga, 2014, Phys. Rev. A 89, 033403.

Ibáñez, S., A. Peralta Conde, D. Guéry-Odelin, and J. G. Muga, 2011, Phys. Rev. A 84, 013428.

Impens, F., and D. Guéry-Odelin, 2017, Phys. Rev. A 96, 043609. Impens, F., and D. Guéry-Odelin, 2019, Sci. Rep. 9, 4048.

Issoufa, Y. H., and A. Messikh, 2014, Phys. Rev. A 90, 055402.

Jaramillo, J., M. Beau, and A. del Campo, 2016, New J. Phys. 18, 075019.

Jarzynski, C., 2013, Phys. Rev. A 88, 040101.

Jarzynski, C., S. Deffner, A. Patra, and Y. Subaşı, 2017, Phys. Rev. E 95, 032122.

Jing, J., L.-A. Wu, M. S. Sarandy, and J. G. Muga, 2013, Phys. Rev. A 88, 053422.

Juliá-Díaz, B., E. Torrontegui, J. Martorell, J. G. Muga, and A. Polls, 2012, Phys. Rev. A 86, 063623.

Kagan, Y., E. L. Surkov, and G. V. Shlyapnikov, 1996, Phys. Rev. A 54, R1753.

Kamsap, M. R., J. Pedregosa-Gutierrez, C. Champenois, D. Guyomarc'h, M. Houssin, and M. Knoop, 2015, Phys. Rev. A 92, 043416. 
Kang, Y.-H., Y.-H. Chen, Z.-C. Shi, B.-H. Huang, J. Song, and Y. Xia, 2017, Phys. Rev. A 96, 022304.

Kang, Y.-H., Y.-H. Chen, Z.-C. Shi, B.-H. Huang, J. Song, and Y. Xia, 2018, Phys. Rev. A 97, 033407.

Kang, Y.-H., Y.-H. Chen, Z.-C. Shi, J. Song, and Y. Xia, 2016a, Phys. Rev. A 94, 052311.

Kang, Y.-H., Y.-H. Chen, Q.-C. Wu, B.-H. Huang, J. Song, and Y. Xia, 2016b, Sci. Rep. 6, 36737.

Kang, Y.-H., Y.-H. Chen, Q.-C. Wu, B.-H. Huang, Y. Xia, and J. Song, 2016c, Sci. Rep. 6, 30151.

Kang, Y.-H., B.-H. Huang, P.-M. Lu, and Y. Xia, 2017, Laser Phys. Lett. 14, 025201.

Kang, Y.-H., Z.-C. Shi, B.-H. Huang, J. Song, and Y. Xia, 2017, Ann. Phys. (Berlin) 529, 1700154.

Karzig, T., F. Pientka, G. Refael, and F. von Oppen, 2015, Phys. Rev. B 91, 201102.

Kastberg, A., W. D. Phillips, S. L. Rolston, R. J. C. Spreeuw, and P. S. Jessen, 1995, Phys. Rev. Lett. 74, 1542.

Kato, T., 1950, J. Phys. Soc. Jpn. 5, 435.

Kaufmann, H., T. Ruster, C. T. Schmiegelow, F. Schmidt-Kaler, and U. G. Poschinger, 2014, New J. Phys. 16, 073012.

Kaufmann, P., T. F. Gloger, D. Kaufmann, M. Johanning, and C. Wunderlich, 2018, Phys. Rev. Lett. 120, 010501.

Khantoul, B., A. Bounames, and M. Maamache, 2017, Eur. Phys. J. Plus 132, 258.

Khomitsky, D. V., L. V. Gulyaev, and E. Y. Sherman, 2012, Phys. Rev. B 85, 125312.

Khujakulov, A., and K. Nakamura, 2016, Phys. Rev. A 93, 022101. Kieferová, M., and N. Wiebe, 2014, New J. Phys. 16, 123034.

Kielpinski, D., C. Monroe, and D. J. Wineland, 2002, Nature (London) 417, 709.

Kiely, A., A. Benseny, T. Busch, and A. Ruschhaupt, 2016, J. Phys. B 49, 215003.

Kiely, A., J. P. L. McGuinness, J. G. Muga, and A. Ruschhaupt, 2015, J. Phys. B 48, 075503.

Kiely, A., J. G. Muga, and A. Ruschhaupt, 2017, Phys. Rev. A 95, 012115.

Kiely, A., J. G. Muga, and A. Ruschhaupt, 2018, Phys. Rev. A 98, 053616.

Kiely, A., and A. Ruschhaupt, 2014, J. Phys. B 47, 115501.

Kiely, A., and A. Ruschhaupt, 2019, unpublished.

Kirk, R. E., 2004, Optimal Control Theory An Introduction (Dover Publications, New York).

Kleißler, F., A. Lazariev, and S. Arroyo-Camejo, 2018, npj Quantum Inf. 4, 49.

Koch, C. P., 2016, J. Phys. Condens. Matter 28, 213001.

Kölbl, J., A. Barfuss, M. S. Kasperczyk, L. Thiel, A. A. Clerk, H. Ribeiro, and P. Maletinsky, 2019, Phys. Rev. Lett. 122, 090502.

Kolodrubetz, M., D. Sels, P. Mehta, and A. Polkovnikov, 2017, Phys. Rep. 697, 1.

Kosloff, R., 2013, Entropy 15, 2100.

Kosloff, R., and Y. Rezek, 2017, Entropy 19, 136.

Kuo, T.-Y. T., and S.-C. J. Kang, 2014, Automation in Construction 42, 25.

Kyaw, T. H., and L.-C. Kwek, 2018, New J. Phys. 20, 045007.

Lakehal, H., M. Maamache, and J. R. Choi, 2016, Sci. Rep. 6, 19860.

Landauer, R., 1961, IBM J. Res. Dev. 5, 183.

Lax, P. D., 1968, Commun. Pure Appl. Math. 21, 467.

Levy, A., A. Kiely, J. G. Muga, R. Kosloff, and E. Torrontegui, 2018, New J. Phys. 20, 025006.

Levy, A., E. Torrontegui, and R. Kosloff, 2017, Phys. Rev. A 96, 033417.
Lewis, H. R., and P. G. L. Leach, 1982, J. Math. Phys. (N.Y.) 23, 2371.

Lewis, Jr., H. R., and W. Riesenfeld, 1969, J. Math. Phys. (N.Y.) 10, 1458.

Li, G., H. T. Quan, and Z. C. Tu, 2017, Phys. Rev. E 96, 012144.

Li, G.-Q., G.-D. Chen, P. Peng, and W. Qi, 2017, Eur. Phys. J. D 71, 14

Li, H., H. Z. Shen, S. L. Wu, and X. X. Yi, 2017, Opt. Express 25, 30135.

Li, J., T. Fogarty, S. Campbell, X. Chen, and T. Busch, 2018, New J. Phys. 20, 015005.

Li, J., K. Sun, and X. Chen, 2016, Sci. Rep. 6, 38258.

Li, J., Q. Zhang, and X. Chen, 2017, Phys. Lett. A 381, 3272.

Li, Y., L.-A. Wu, and Z. D. Wang, 2011, Phys. Rev. A 83, 043804.

Li, Y.-C., and X. Chen, 2016, Phys. Rev. A 94, 063411.

Li, Y.-C., X. Chen, J. G. Muga, and E. Y. Sherman, 2018, New J. Phys. 20, 113029.

Li, Y.-C., D. Martínez-Cercós, S. Martínez-Garaot, X. Chen, and J. G. Muga, 2018, Phys. Rev. A 97, 013830.

Liang, Y., X. Ji, H.-F. Wang, and S. Zhang, 2015, Laser Phys. Lett. 12, 115201.

Liang, Y., C. Song, X. Ji, and S. Zhang, 2015, Opt. Express 23, 23798.

Liang, Y., S.-L. Su, Q.-C. Wu, X. Ji, and S. Zhang, 2015, Opt. Express 23, 5064.

Liang, Y., Q.-C. Wu, S.-L. Su, X. Ji, and S. Zhang, 2015, Phys. Rev. A 91, 032304.

Liang, Z.-T., X. Yue, Q. Lv, Y.-X. Du, W. Huang, H. Yan, and S.-L. Zhu, 2016, Phys. Rev. A 93, 040305.

Lin, J.-B., Y. Liang, C. Song, X. Ji, and S. Zhang, 2016, J. Opt. Soc. Am. B 33, 519.

Lin, T.-Y., F.-C. Hsiao, Y.-W. Jhang, C. Hu, and S.-Y. Tseng, 2012, Opt. Express 20, 24085.

Liu, B.-J., Z.-H. Huang, Z.-Y. Xue, and X.-D. Zhang, 2017, Phys. Rev. A 95, 062308.

Liu, B.-J., X.-K. Song, Z.-Y. Xue, X. Wang, and M.-H. Yung, 2018, arXiv:1806.07904.

Liu, Y.-H., and S.-Y. Tseng, 2017, J. Phys. B 50, 205501.

Lizuain, I., M. Palmero, and J. G. Muga, 2017, Phys. Rev. A 95, 022130 .

Lizuain, I., A. Tobalina, A. Rodriguez-Prieto, and J. G. Muga, 2019, arXiv:1904.08987v2.

Lohe, M. A., 2009, J. Phys. A 42, 035307.

Longhi, S., 2009, Laser Photonics Rev. 3, 243.

Longhi, S., 2017, Phys. Rev. A 95, 062122.

Lu, M., Y. Xia, L.-T. Shen, and J. Song, 2014, Laser Phys. 24, 105201.

Lu, M., Y. Xia, L.-T. Shen, J. Song, and N. B. An, 2014, Phys. Rev. A 89, 012326.

Lu, X.-J., X. Chen, J. Alonso, and J. G. Muga, 2014, Phys. Rev. A 89, 023627.

Lu, X.-J., X. Chen, A. Ruschhaupt, D. Alonso, S. Guérin, and J. G. Muga, 2013, Phys. Rev. A 88, 033406.

Lu, X.-J., M. Li, Z. Y. Zhao, C.-L. Zhang, H.-P. Han, Z.-B. Feng, and Y.-Q. Zhou, 2017, Phys. Rev. A 96, 023843.

Lu, X.-J., J. G. Muga, X. Chen, U. G. Poschinger, F. Schmidt-Kaler, and A. Ruschhaupt, 2014, Phys. Rev. A 89, 063414.

Lu, X.-J., M. Palmero, A. Ruschhaupt, X. Chen, and J. G. Muga, 2015, Phys. Scr. 90, 074038.

Lu, X.-J., A. Ruschhaupt, and J. G. Muga, 2018, Phys. Rev. A 97, 053402 .

Luo, D.-W., P. V. Pyshkin, C.-H. Lam, T. Yu, H.-Q. Lin, J. Q. You, and L.-A. Wu, 2015, Phys. Rev. A 92, 062127. 
Lv, Q.-X., H.-Z. Liu, J.-H. Liang, K.-Y. Liao, and Y.-X. Du, 2019, arXiv:1903.03397.

Ma, W., X. J. Cao, and S. L. Wu, 2017, Opt. Express 25, 23850.

Maamache, M., O. Kaltoum Djeghiour, N. Mana, and W. Koussa, 2017, Eur. Phys. J. Plus 132, 383.

Mandelstam, L., and I. Tamm, 1945, J. Phys. USSR 9, 249.

Marr, C., A. Beige, and G. Rempe, 2003, Phys. Rev. A 68, 033817.

Martínez, I. A., A. Petrosyan, D. Guéry-Odelin, E. Trizac, and S. Ciliberto, 2016, Nat. Phys. 12, 843.

Martínez, I. A., E. Roldán, J. M. R. Parrondo, and D. Petrov, 2013, Phys. Rev. E 87, 032159.

Martínez-Garaot, S., J. G. Muga, and S.-Y. Tseng, 2017, Opt. Express 25, 159.

Martínez-Garaot, S., M. Palmero, D. Guéry-Odelin, and J. G. Muga, 2015, Phys. Rev. A 92, 053406.

Martínez-Garaot, S., M. Palmero, J. G. Muga, and D. Guéry-Odelin, 2016, Phys. Rev. A 94, 063418.

Martínez-Garaot, S., A. Rodriguez-Prieto, and J. G. Muga, 2018, Phys. Rev. A 98, 043622.

Martínez-Garaot, S., A. Ruschhaupt, J. Gillet, T. Busch, and J. G. Muga, 2015, Phys. Rev. A 92, 043406.

Martínez-Garaot, S., E. Torrontegui, X. Chen, M. Modugno, D. Guéry-Odelin, S.-Y. Tseng, and J. G. Muga, 2013, Phys. Rev. Lett. 111, 213001.

Martínez-Garaot, S., E. Torrontegui, X. Chen, and J. G. Muga, 2014, Phys. Rev. A 89, 053408.

Martínez-Garaot, S., S.-Y. Tseng, and J. G. Muga, 2014, Opt. Lett. 39, 2306.

Martinis, J. M., and M. R. Geller, 2014, Phys. Rev. A 90, 022307.

Masuda, S., and K. Nakamura, 2008, Phys. Rev. A 78, 062108.

Masuda, S., and K. Nakamura, 2010, Proc. R. Soc. A 466, 1135.

Masuda, S., and K. Nakamura, 2011, Phys. Rev. A 84, 043434.

Masuda, S., K. Nakamura, and A. del Campo, 2014, Phys. Rev. Lett. 113, 063003.

Masuda, S., K. Nakamura, and M. Nakahara, 2018, New J. Phys. 20, 025008.

Masuda, S., and S. A. Rice, 2014, Phys. Rev. A 89, 033621.

Masuda, S., and S. A. Rice, 2015a, J. Phys. Chem. A 119, 3479.

Masuda, S., and S. A. Rice, 2015b, J. Chem. Phys. 142, 244303.

Masuda, S., and S. A. Rice, 2015c, J. Phys. Chem. B 119, 11079.

Masuda, S., and S. A. Rice, 2016, Adv. Chem. Phys. 159, 51.

Menchon-Enrich, R., A. Benseny, V. Ahufinger, A. D. Greentree, T. Busch, and J. Mompart, 2016, Rep. Prog. Phys. 79, 074401.

Messiah, A., 1962, Quantum Mechanics, Vol. II (North-Holland, Amsterdam).

Mishima, H., and Y. Izumida, 2017, Phys. Rev. E 96, 012133.

Morita, S., 2007, J. Phys. Soc. Jpn. 76, 104001.

Mortensen, H. L., J. J. Sørensen, K. Mølmer, and J. F. Sherson, 2018, New J. Phys. 20, 025009.

Motzoi, F., J. M. Gambetta, P. Rebentrost, and F. K. Wilhelm, 2009, Phys. Rev. Lett. 103, 110501.

Muga, J., J. Palao, B. Navarro, and I. Egusquiza, 2004, Phys. Rep. 395, 357.

Muga, J. G., X. Chen, S. Ibáñez, I. Lizuain, and A. Ruschhaupt, 2010, J. Phys. B 43, 085509.

Muga, J. G., X. Chen, A. Ruschhaupt, and D. Guéry-Odelin, 2009, J. Phys. B 42, 241001.

Muga, J. G., J. Echanobe, A. del Campo, and I. Lizuain, 2008, J. Phys. B 41, 175501.

Muga, J. G., M. A. Simón, and A. Tobalina, 2016, New J. Phys. 18, 021005.

Mukherjee, V., S. Montangero, and R. Fazio, 2016, Phys. Rev. A 93, 062108.
Nakamura, K., A. Khujakulov, S. Avazbaev, and S. Masuda, 2017, Phys. Rev. A 95, 062108.

Navez, P., S. Pandey, H. Mas, K. Poulios, T. Fernholz, and W. von Klitzing, 2016, New J. Phys. 18, 075014.

Ndong, M., G. Djotyan, A. Ruschhaupt, and S. Gurin, 2015, J. Phys. B 48, 174007.

Nenciu, G., and G. Rasche, 1992, J. Phys. A 25, 5741.

Ness, G., C. Shkedrov, Y. Florshaim, and Y. Sagi, 2018, New J. Phys. 20, 095002.

Nishimura, K., and K. Takahashi, 2018, SciPost Phys. 5, 29.

Nizamani, A. H., and W. K. Hensinger, 2012, Appl. Phys. B 106, 327.

Oh, S., and S. Kais, 2014, J. Chem. Phys. 141, 224108.

Okuyama, M., and K. Takahashi, 2016, Phys. Rev. Lett. 117, 070401.

Okuyama, M., and K. Takahashi, 2017, J. Phys. Soc. Jpn. 86, 043002.

Onofrio, R., 2016, Phys. Usp. 59, 1129.

Opatrný, T., and K. Mølmer, 2014, New J. Phys. 16, 015025.

Opatrný, T., H. Saberi, E. Brion, and K. Mølmer, 2016, Phys. Rev. A 93, 023815.

Oreshkov, O., and J. Calsamiglia, 2010, Phys. Rev. Lett. 105, 050503.

Ozcakmakli, Z., and C. Yuce, 2012, Phys. Scr. 86, 055001.

Palmero, M., R. Bowler, J. P. Gaebler, D. Leibfried, and J. G. Muga, 2014, Phys. Rev. A 90, 053408.

Palmero, M., S. Martínez-Garaot, J. Alonso, J. P. Home, and J. G. Muga, 2015, Phys. Rev. A 91, 053411.

Palmero, M., S. Martínez-Garaot, D. Leibfried, D. J. Wineland, and J. G. Muga, 2017, Phys. Rev. A 95, 022328.

Palmero, M., S. Martínez-Garaot, U. G. Poschinger, A. Ruschhaupt, and J. G. Muga, 2015, New J. Phys. 17, 093031.

Palmero, M., E. Torrontegui, D. Guéry-Odelin, and J. G. Muga, 2013, Phys. Rev. A 88, 053423.

Palmero, M., S. Wang, D. Guéry-Odelin, J.-S. Li, and J. G. Muga, 2016, New J. Phys. 18, 043014.

Pan, T.-H., and S.-Y. Tseng, 2015, Opt. Express 23, 10405.

Pang, S., and A. N. Jordan, 2017, Nat. Commun. 8, 14695.

Papoular, D. J., and S. Stringari, 2015, Phys. Rev. Lett. 115, 025302.

Patra, A., and C. Jarzynski, 2017a, J. Phys. Chem. B 121, 3403.

Patra, A., and C. Jarzynski, 2017b, New J. Phys. 19, 125009.

Paul, K., and A. K. Sarma, 2015, Phys. Rev. A 91, 053406.

Pedregosa-Gutierrez, J., C. Champenois, M. R. Kamsap, and M. Knoop, 2015, Int. J. Mass Spectrom. 381-382, 33.

Pekola, J. P., V. Brosco, M. Möttönen, P. Solinas, and A. Shnirman, 2010, Phys. Rev. Lett. 105, 030401.

Peng, R., Y. Zheng, S.-W. Liu, X.-P. Li, J.-L. Wu, and X. Ji, 2017, Quantum Inf. Process. 16, 172.

Petiziol, F., B. Dive, S. Carretta, R. Mannella, F. Mintert, and S. Wimberger, 2019, arXiv:1901.07344.

Petiziol, F., B. Dive, F. Mintert, and S. Wimberger, 2018, Phys. Rev. A 98, 043436.

Pinch, E. R., 1993, Optimal Control and the Calculus of Variations (Oxford University Press, New York).

Plata, C. A., D. Guéry-Odelin, E. Trizac, and A. Prados, 2019, Phys. Rev. E 99, 012140.

Puri, S., S. Boutin, and A. Blais, 2017, npj Quantum Inf. 3, 18. Qi, S., and J. Jing, 2019, arXiv:1905.09524.

Quan, H. T., and W. H. Zurek, 2010, New J. Phys. 12, 093025.

Ran, D., Z.-C. Shi, J. Song, and Y. Xia, 2017, Phys. Rev. A 96, 033803.

Reichle, R., D. Leibfried, R. B. Blakestad, J. Britton, J. D. Jost, E. Knill, C. Langer, R. Ozeri, S. Seidelin, and D. J. Wineland, 2006, Fortschritte der Physik: Progress of Physics 54, 666. 
Ren, F.-H., Z.-M. Wang, and Y.-J. Gu, 2017, Phys. Lett. A 381, 70. Rezakhani, A. T., A. K. Pimachev, and D. A. Lidar, 2010, Phys. Rev. A 82, 052305.

Rezek, Y., P. Salamon, K. H. Hoffmann, and R. Kosloff, 2009, Europhys. Lett. 85, 30008.

Richerme, P., C. Senko, J. Smith, A. Lee, S. Korenblit, and C. Monroe, 2013, Phys. Rev. A 88, 012334.

Ritland, K., and A. Rahmani, 2018, New J. Phys. 20, 065005.

Rohringer, W., D. Fischer, F. Steiner, I. E. Mazets, J. Schmiedmayer, and M. Trupke, 2015, Sci. Rep. 5, 9820.

Roland, J., and N. J. Cerf, 2002, Phys. Rev. A 65, 042308.

Rouchon, P., 2005, ZAMM-Journal of Applied Mathematics and Mechanics/Zeitschrift für Angewandte Mathematik und Mechanik 85, 411.

Ruschhaupt, A., X. Chen, D. Alonso, and J. G. Muga, 2012, New J. Phys. 14, 093040.

Ruschhaupt, A., and J. Muga, 2014, J. Mod. Opt. 61, 828.

Ruster, T., C. Warschburger, H. Kaufmann, C. T. Schmiegelow, A. Walther, M. Hettrich, A. Pfister, V. Kaushal, F. Schmidt-Kaler, and U. G. Poschinger, 2014, Phys. Rev. A 90, 033410.

Saberi, H., T. Opatrný, K. Mølmer, and A. del Campo, 2014, Phys. Rev. A 90, 060301.

Salamon, P., K. H. Hoffmann, Y. Rezek, and R. Kosloff, 2009, Phys. Chem. Chem. Phys. 11, 1027.

Santos, A. C., 2018, J. Phys. B 51, 015501.

Santos, A. C., A. Nicotina, A. M. de Souza, R. S. Sarthour, I. S. Oliveira, and M. S. Sarandy, 2019, arXiv:1906.08065.

Santos, A. C., and M. S. Sarandy, 2015, Sci. Rep. 5, 15775.

Santos, A. C., and M. S. Sarandy, 2018, J. Phys. A 51, 025301.

Santos, A. C., R. D. Silva, and M. S. Sarandy, 2016, Phys. Rev. A 93, 012311.

Sarandy, M., E. Duzzioni, and R. Serra, 2011, Phys. Lett. A 375, 3343.

Sarandy, M. S., E. I. Duzzioni, and M. H. Y. Moussa, 2007, Phys. Rev. A 76, 052112.

Sarandy, M. S., and D. A. Lidar, 2005, Phys. Rev. A 71, 012331.

Schaff, J.-F., P. Capuzzi, G. Labeyrie, and P. Vignolo, 2011, New J. Phys. 13, 113017.

Schaff, J.-F., X.-L. Song, P. Capuzzi, P. Vignolo, and G. Labeyrie, 2011, Europhys. Lett. 93, 23001.

Schaff, J.-F., X.-L. Song, P. Vignolo, and G. Labeyrie, 2010, Phys. Rev. A 82, 033430.

Schaller, G., S. Mostame, and R. Schützhold, 2006, Phys. Rev. A 73, 062307.

Schloss, J., A. Benseny, J. Gillet, J. Swain, and T. Busch, 2016, New J. Phys. 18, 035012.

Schmiedl, T., E. Dieterich, P.-S. Dieterich, and U. Seifert, 2009, J. Stat. Mech. 07, P07013.

Schutjens, R., F. A. Dagga, D. J. Egger, and F. K. Wilhelm, 2013, Phys. Rev. A 88, 052330.

Sekimoto, K., and S.-i. Sasa, 1997, J. Phys. Soc. Jpn. 66, 3326.

Sels, D., and A. Polkovnikov, 2017, Proc. Natl. Acad. Sci. U.S.A. 114, E3909.

Setiawan, I., B. E. Gunara, S. Avazbaev, and K. Nakamura, 2019, Phys. Rev. A 99, 062116.

Setiawan, I., B. E. Gunara, S. Masuda, and K. Nakamura, 2017, Phys. Rev. A 96, 052106.

Shan, W.-J., Y. Xia, Y.-H. Chen, and J. Song, 2016, Quantum Inf. Process. 15, 2359.

Shen, C.-P., and S. Su, 2018, arXiv:1807.02808.

Shi, X., and L. F. Wei, 2015, Laser Phys. Lett. 12, 015204.

Simón, M. Á., Á. Buendía, and J. G. Muga, 2018, Mathematics 6, 111.
Smith, A., Y. Lu, S. An, X. Zhang, J.-N. Zhang, Z. Gong, H. T. Quan, C. Jarzynski, and K. Kim, 2018, New J. Phys. 20, 013008.

Song, C., S.-L. Su, C.-H. Bai, X. Ji, and S. Zhang, 2016, Quantum Inf. Process. 15, 4159.

Song, J., Z.-J. Zhang, Y. Xia, X.-D. Sun, and Y.-Y. Jiang, 2016, Opt. Express 24, 21674.

Song, K.-H., and M.-F. Chen, 2016, Quantum Inf. Process. 15, 3169.

Song, L.-C., Y. Xia, and J. Song, 2014, J. Mod. Opt. 61, 1290.

Song, X.-K., Q. Ai, J. Qiu, and F.-G. Deng, 2016, Phys. Rev. A 93, 052324.

Song, X.-K., F.-G. Deng, L. Lamata, and J. G. Muga, 2017, Phys. Rev. A 95, 022332.

Song, X.-K., H. Zhang, Q. Ai, J. Qiu, and F.-G. Deng, 2016d, New J. Phys. 18, 023001.

Stefanatos, D., 2013, Automatica 49, 3079.

Stefanatos, D., 2014, Phys. Rev. A 90, 023811.

Stefanatos, D., 2017a, Phys. Rev. E 96, 042103.

Stefanatos, D., 2017b, IEEE Trans. Autom. Control 62, 4290.

Stefanatos, D., 2018, J. Appl. Math. Bioinformatics 8, 17.

Stefanatos, D., and J.-S. Li, 2014, IEEE Trans. Autom. Control 59, 733.

Stefanatos, D., and E. Paspalakis, 2018a, arXiv:1805.07129.

Stefanatos, D., and E. Paspalakis, 2018b, New J. Phys. 20, 055009.

Stefanatos, D., and E. Paspalakis, 2019a, Phys. Rev. A 100, 012111.

Stefanatos, D., and E. Paspalakis, 2019b, arXiv:1907.00166.

Stefanatos, D., J. Ruths, and J.-S. Li, 2010, Phys. Rev. A 82, 063422.

Sun, C., A. Saxena, and N. A. Sinitsyn, 2017, J. Phys. B 50, 175501.

Sun, N., X. Zhang, Y. Fang, and Y. Yuan, 2012, IET Control Theory \& Applications 6, 1410.

Sun, Z., L. Zhou, G. Xiao, D. Poletti, and J. Gong, 2016, Phys. Rev. A 93, 012121.

Takahashi, K., 2013a, J. Phys. A 46, 315304.

Takahashi, K., 2013b, Phys. Rev. E 87, 062117.

Takahashi, K., 2014, Phys. Rev. A 89, 042113.

Takahashi, K., 2015, Phys. Rev. A 91, 042115.

Takahashi, K., 2017a, New J. Phys. 19, 115007.

Takahashi, K., 2017b, Phys. Rev. A 95, 012309.

Takahashi, K., 2019, J. Phys. Soc. Jpn. 88, 061002.

Takahashi, K., and M. Ohzeki, 2016, Phys. Rev. E 93, 012129.

Theis, L. S., F. Motzoi, S. Machnes, and F. K. Wilhelm, 2018, Europhys. Lett. 123, 60001.

Theis, L. S., F. Motzoi, and F. K. Wilhelm, 2016, Phys. Rev. A 93, 012324.

Thunström, P., J. Åberg, and E. Sjöqvist, 2005, Phys. Rev. A 72, 022328.

Tian, L., 2018, arXiv:1802.02285.

Tobalina, A., J. Alonso, and J. G. Muga, 2018, New J. Phys. 20, 065002.

Tobalina, A., I. Lizuain, and J. G. Muga, 2019, arXiv:1906.07473.

Tobalina, A., M. Palmero, S. Martínez-Garaot, and J. G. Muga, 2017, Sci. Rep. 7, 5753.

Torosov, B. T., G. Della Valle, and S. Longhi, 2013, Phys. Rev. A 87, 052502.

Torosov, B. T., G. Della Valle, and S. Longhi, 2014, Phys. Rev. A 89, 063412

Torrontegui, E., X. Chen, M. Modugno, A. Ruschhaupt, D. GuéryOdelin, and J. G. Muga, 2012, Phys. Rev. A 85, 033605.

Torrontegui, E., X. Chen, M. Modugno, S. Schmidt, A. Ruschhaupt, and J. G. Muga, 2012, New J. Phys. 14, 013031.

Torrontegui, E., S. Dawkins, M. Göb, and K. Singer, 2018, New J. Phys. 20, 105001.

Torrontegui, E., and J. J. García-Ripoll, 2019, Europhys. Lett. 125, 30004. 
Torrontegui, E., S. Ibáñez, X. Chen, A. Ruschhaupt, D. GuéryOdelin, and J. G. Muga, 2011, Phys. Rev. A 83, 013415.

Torrontegui, E., S. Ibáñez, S. Martínez-Garaot, M. Modugno, A. del Campo, D. Guéry-Odelin, A. Ruschhaupt, X. Chen, and J. G. Muga, 2013, Adv. At. Mol. Opt. Phys. 62, 117.

Torrontegui, E., and R. Kosloff, 2013, Phys. Rev. E 88, 032103.

Torrontegui, E., I. Lizuain, S. González-Resines, A. Tobalina, A. Ruschhaupt, R. Kosloff, and J. G. Muga, 2017, Phys. Rev. A 96, 022133.

Torrontegui, E., S. Martínez-Garaot, M. Modugno, X. Chen, and J. G. Muga, 2013, Phys. Rev. A 87, 033630.

Torrontegui, E., S. Martínez-Garaot, and J. G. Muga, 2014, Phys. Rev. A 89, 043408.

Torrontegui, E., S. Martínez-Garaot, A. Ruschhaupt, and J. G. Muga, 2012, Phys. Rev. A 86, 013601.

Tseng, S.-Y., 2013, Opt. Express 21, 21224.

Tseng, S.-Y., 2014, Opt. Lett. 39, 6600.

Tseng, S.-Y., and X. Chen, 2012, Opt. Lett. 37, 5118.

Tseng, S.-Y., R.-D. Wen, Y.-F. Chiu, and X. Chen, 2014, Opt. Express 22, 18849.

Tu, Z. C., 2014, Phys. Rev. E 89, 052148.

Unanyan, R. G., L. P. Yatsenko, K. Bergmann, and B. W. Shore, 1997, Opt. Commun. 139, 48.

Vacanti, G., R. Fazio, S. Montangero, G. M. Palma, M. Paternostro, and V. Vedral, 2014, New J. Phys. 16, 053017.

Van-Damme, L., D. Schraft, G. T. Genov, D. Sugny, T. Halfmann, and S. Guérin, 2017, Phys. Rev. A 96, 022309.

Venuti, L. C., T. Albash, D. A. Lidar, and P. Zanardi, 2016, Phys. Rev. A 93, 032118.

Vepsäläinen, A., S. Danilin, and G. Paraoanu, 2018, Quant. Sci. Technol. 3, 024006.

Vepsäläinen, A., S. Danilin, and G. S. Paraoanu, 2019, Sci. Adv. 5, eaau5999.

Villazon, T., A. Polkovnikov, and A. Chandran, 2019, arXiv: 1902.05964.

Vitanov, N. V., and M. Drewsen, 2019, Phys. Rev. Lett. 122, 173202.

Vitanov, N. V., A. A. Rangelov, B. W. Shore, and K. Bergmann, 2017, Rev. Mod. Phys. 89, 015006.

Vitanov, N. V., and B. W. Shore, 2015, J. Phys. B 48, 174008.

Walther, A., F. Ziesel, T. Ruster, S. T. Dawkins, K. Ott, M. Hettrich, K. Singer, F. Schmidt-Kaler, and U. Poschinger, 2012, Phys. Rev. Lett. 109, 080501.

Wang, T., et al., 2018, New J. Phys. 20, 065003.

Wang, Z., Y. Xia, Y.-H. Chen, and J. Song, 2016, Eur. Phys. J. D 70, 162.

Wang, Z.-M., C. A. Bishop, J. Jing, Y.-J. Gu, C. Garcia, and L.-A. Wu, 2016, Phys. Rev. A 93, 062338.

Wendin, G., 2017, Rep. Prog. Phys. 80, 106001.

Wiebe, N., and N. S. Babcock, 2012, New J. Phys. 14, 013024.

Wineland, D. J., C. Monroe, W. M. Itano, D. Leibfried, B. E. King, and D. M. Meekhof, 1998, J. Res. Natl. Inst. Stand. Technol. 103, 259.

Wu, J.-L., X. Ji, and S. Zhang, 2016, Sci. Rep. 6, 33669.

Wu, J.-L., X. Ji, and S. Zhang, 2017a, Quantum Inf. Process. 16, 294.

Wu, J.-L., X. Ji, and S. Zhang, 2017b, Sci. Rep. 7, 46255.

Wu, J.-L., X. Ji, and S. Zhang, 2017c, Opt. Express 25, 21084.

Wu, J.-L., C. Song, X. Ji, and S. Zhang, 2016, J. Opt. Soc. Am. B 33, 2026.

Wu, J.-L., S.-L. Su, X. Ji, and S. Zhang, 2017, Ann. Phys. (Amsterdam) 386, 34.
Wu, Q.-C., Y.-H. Chen, B.-H. Huang, J. Song, Y. Xia, and S.-B. Zheng, 2016, Opt. Express 24, 22847.

Wu, S. L., X. L. Huang, H. Li, and X. X. Yi, 2017, Phys. Rev. A 96, 042104.

Wu, S. L., X. Y. Zhang, and X. X. Yi, 2015, Phys. Rev. A 92, 062122.

Xiao, G., and J. Gong, 2014, Phys. Rev. E 90, 052132.

Xing-Xin, C., Z. Jun, N. Xi-Jing, and Z. Wen-Xian, 2013, Chin. Phys. B 22, 090310.

Xu, J., L. Yu, J.-L. Wu, and X. Ji, 2017, Chin. Phys. B 26, 090301.

Yan, T., et al., 2019, Phys. Rev. Lett. 122, 080501.

Yan, Y., Y. Li, A. Kinos, A. Walther, C. Shi, L. Rippe, J. Moser, S. Kröll, and X. Chen, 2019, Opt. Express 27, 8267.

Yang, Y.-F., Y.-H. Chen, Q.-C. Wu, Y.-H. Kang, B.-H. Huang, and Y. Xia, 2017, Quantum Inf. Process. 16, 15.

Yang, Y.-F., Y.-H. Chen, Q.-C. Wu, Z.-C. Shi, J. Song, and Y. Xia, 2018, Can. J. Phys. 96, 81.

Ye, L.-X., X. Lin, X. Chen, J. He, R.-C. Yang, and H.-Y. Liu, 2016, Quantum Inf. Process. 15, 2785.

Yeih, C.-S., H.-X. Cao, and S.-Y. Tseng, 2014, IEEE Photonics Technol. Lett. 26, 123.

Yi, X. X., D. M. Tong, L. C. Kwek, and C. H. Oh, 2007, J. Phys. B 40, 281.

Yoshimura, B., and J. K. Freericks, 2015, Front. Phys. 3, 85.

Yu, L., J. Xu, J.-L. Wu, and X. Ji, 2017, Chin. Phys. B 26, 060306.

Yu, X.-T., Q. Zhang, Y. Ban, and X. Chen, 2018, Phys. Rev. A 97, 062317.

Yuce, C., 2012, Phys. Lett. A 376, 1717.

Yuste, A., B. Juliá-Díaz, E. Torrontegui, J. Martorell, J. G. Muga, and A. Polls, 2013, Phys. Rev. A 88, 043647.

Zhang, H., X.-K. Song, Q. Ai, H. Wang, G.-J. Yang, and F.-G. Deng, 2018, arXiv:1809.00102.

Zhang, J., T. H. Kyaw, D. M. Tong, E. Sjöqvist, and L.-C. Kwek, 2015, Sci. Rep. 5, 18414.

Zhang, J., F.-G. Li, Y. Xie, C.-W. Wu, B.-Q. Ou, W. Wu, and P.-X. Chen, 2018, Phys. Rev. A 98, 052323.

Zhang, J., et al., 2013, Phys. Rev. Lett. 110, 240501.

Zhang, J.-Q., Y. Li, and M. Feng, 2013, J. Phys. Condens. Matter 25, 142201.

Zhang, Q., X. Chen, and D. Guéry-Odelin, 2015, Phys. Rev. A 92, 043410

Zhang, Q., X. Chen, and D. Guéry-Odelin, 2017, Sci. Rep. 7, 15814.

Zhang, Q., J. G. Muga, D. Guéry-Odelin, and X. Chen, 2016, J. Phys. B 49, 125503.

Zhang, X., Y.-H. Chen, Q.-C. Wu, Z.-C. Shi, J. Song, and Y. Xia, 2017, Laser Phys. 27, 015202.

Zhang, Z., T. Wang, L. Xiang, J. Yao, J. Wu, and Y. Yin, 2017, Phys. Rev. A 95, 042345.

Zhang, Z., et al., 2018, New J. Phys. 20, 085001.

Zhao, Y. J., B. Liu, Y. Q. Ji, S. Q. Tang, and X. Q. Shao, 2017, Sci. Rep. 7, 16489.

Zheng, Y., S. Campbell, G. De Chiara, and D. Poletti, 2016, Phys. Rev. A 94, 042132.

Zhong, Z.-R., 2016, Sci. Rep. 6, 8.

Zhou, B. B., A. Baksic, H. Ribeiro, C. G. Yale, F. J. Heremans, P. C. Jerger, A. Auer, G. Burkard, A. A. Clerk, and D. D. Awschalom, 2017, Nat. Phys. 13, 330.

Zhou, X., S. Jin, and J. Schmiedmayer, 2018, New J. Phys. 20, 055005.

Zhou, X., B.-J. Liu, L. Shao, X.-D. Zhang, and Z.-Y. Xue, 2017, Laser Phys. Lett. 14, 095202. 